\title{
Interlocking directorates in Spanish banking in the twentieth century
}

\author{
Javier Pueyo \\ Universitat Pompeu Fabra \\ Dpto. de Economia i Empresa \\ Ramon Trias Fargas, 25-27 \\ 08005 Barcelona \\ javier.pueyo@upf.edu
}

\begin{abstract}
Spanish banking historiography asserts that the largest banks performed in the twentieth century as though they constituted a monopoly. One of their main coordination schemes would have been a network of interlocking bank directors that would include most of the financial firms. Evidence available for the 1920s and 1960s seems to confirm the veracity of this hypothesis. In this paper, more systematic evidence is presented to cover the whole twentieth century with the aim of checking whether these networks persisted over the entire period or they were by-products of temporary situations. Our results show that no general network remained for more than a decade. Therefore, it should be ruled out that interlocking directorates worked as a coordination device of an alleged banking cartel.
\end{abstract}

Key words: Monopolization strategies, Interlocking directorates, Spanish banking. JEL codes: L12, L14, N24. 


\section{INDICE}

1. Introducción 3

2. Historiographic debate 4

3. Interlocking directorates in Spanish banking in the twentieth century 6

4. Bank networks in Spanish banking in the twentieth century 7

5. Conclusions 9

$\begin{array}{lr}\text { Bibliography } & 10\end{array}$

$\begin{array}{ll}\text { Appendix 1. Associations of banks } & 12\end{array}$

$\begin{array}{ll}\text { Appendix 2. Bank association's diagrams } & 23\end{array}$

Appendix 3. Diagrams of connections between groups 40

Appendix 4: Lists of interlocking directors 44

Banco Hispano Americano 44

Banco Urquijo $\quad 48$

Banco Español de Crédito (Banesto) $\quad 50$

Banco Central $\quad 54$

Banco de Bilbao $\quad 58$

Banco de Vizcaya $\quad 61$

Catalan banks before the civil war $\quad 64$

Banco de Santander $\quad 67$

$\begin{array}{ll}\text { Banco Popular } & 70\end{array}$

$\begin{array}{ll}\text { Other relations } & 72\end{array}$ 


\section{Introduction}

Spanish historiography about banking asserts that, in the twentieth century, the largest banks behaved as though they constituted a cartel. One of the main coordination mechanisms of this so-called monopoly would be a set of board of directors' members who seated simultaneously in the directorates of many financial firms. These directors would coordinate collusive strategies for restraining competition among banks and collecting monopoly rents. Empirical evidence giving support to this hypothesis was established by interlocking directorate analysis. Two studies about bank relationships applied this technique to recreate networks of banks operating in the 1920 s and the 1960s. In both decades, it was detected a compact web connecting nearly all the large banks and great part of the medium-size banks. This finding was considered by some scholars as a proof in favour of the thesis of permanent collusion in the Spanish banking industry, which would have been running at least since the First World War. ${ }^{1}$

This hypothesis was put forward by a group of scholars associated with the official political party (Falange Española), who criticized a supposed infiltration of big business in Franco's governments and the civil service. Years after, this viewpoint was assumed by groups of the left-wind opposition, who used it to denounce the oligarchic base of the political regime. ${ }^{2}$ The conjecture that the banking industry was shaped as a monopoly was established in view of the opaque relationships between banking firms and the government, which was interpreted with the aid of Hilferding's hypothesis of financial capital and supported by a set of empirical studies. Evidence about connections between banks' directorates and by means of other banking organizations was collected. Despite of the political origin of this hypothesis, it was completely accepted by current Spanish historiography without further research until very recently. ${ }^{3}$

Coordination by interlocking directorates means implicitly that firm's boards of directors should have full capability to decide entrepreneurial strategies. In this case, interlocking directors' functions would include enforcing collusive agreements and coordinating banks' behaviour when needed. This oligopolistic coordination scheme would be in force since the 1920s, when the first Spanish banking regulations were passed. It would survive the civil war and Franco's government regulation would strengthen it. In fact, it would not disappear until the 1980s, once Spain became a member of the European Communities. Scholars supporting collusive hypothesis also pointed to the procedure to provide empirical evidence: interlocking directorate technique of analysis. A network of interlocking bank directors that included most of the financial firms was detected for the 1920s and the 1960s, but no research was developed to check whether this net stayed in the intervening decades. Therefore, it was implicitly supposed that the network endured and collusion would also prevail.

The aim of this paper is to present new evidence about interlocking directorates in Spanish banking in the twentieth century in order to examine whether collusive coordination by this practice was feasible. After a summary of the debate about interlocking directorates in Spanish banking in the twentieth century (section 2), new

\footnotetext{
${ }^{1}$ See Roldán-García Delgado-Muñoz (1973) and Muñoz (1969). Pueyo (forthcoming) provides a survey about the origin and development of this hypothesis.

${ }^{2}$ For the falangist affiliation, see a compilation of editorials and articles previously published by Arriba (AA.VV., 1954) and, for the connection with the leftist opposition, see two extra issues of Cuadernos para el Diálogo, published in 1968. The most outstanding papers from the latter reference served to compound Muñoz (1969).

${ }^{3}$ Banking cartelisation is the recognised hypothesis in textbooks: see, for instance, Tortella (2000). Recently, new research has challenged current interpretation: see Pons (1999, 2001, 2002), García Ruiz (2002), Garc ía Ruiz-Tortella (2003), and Pueyo (2003).
} 
empirical evidence is displayed (section 3 and appendixes). Lists of board of directors were gathered up for a sample of Spanish banks in 1915, 1925, 1934 and 1947, and for the whole private banks in 1975, 1980 and 1985. Our results are compared with those of previous studies (1921, 1961 and 1966-67) to check the persistence of interlocking directorates networks. Section 4 is devoted to some comments, stressing that new evidence works against the collusive hypothesis. A section of conclusions closed the paper.

\section{Historiographic debate}

Current literature about the history of Spanish banking in the twentieth century asserts that this industry was organized as a cartel, headed by the largest banks. In fact, "monopoly" is the word usually employed in these studies to characterize financial firms' relationships. Thus, scholars stressed the state of permanent collusion in Spanish financial markets since the end of First World War until the last decades of the twentieth century. Origin of this interpretation can be found in a number of researches carried out in the 1940s and 1950s. They analized market structure in some industries looking for conditions that facilitate agreements to restrict competition. ${ }^{4}$ According to contemporary concepts and available sources, it was established a list of industries with apparent oligopolistic features. Analysis examined concentration indexes, height of entry barriers, and inter-firm connections making use of interlocking directorates methodology.

Banking industry was in the list of activities with high probability of collusive behaviour. Quoted studies established that economic concentration was high, entry into the industry was absolutely blocked by status quo regulation and interlocking directorates closely tied large banks. This latter feature was interpreted as strong evidence of cooperative behaviour. Besides, the semi-official banking association, Consejo Superior Bancario (CSB), and the boards of directors of the Bank of Spain and other official banks were also employed as meeting places to discuss and decide strategies for the whole banking industry. On the basis of this evidence, it was established the hypothesis that Spanish banking industry was structured as a perfect collusive oligopoly with government support. Origin of the cartel was dated in the 1920s, when the first banking law was enacted (1921), CSB was instituted and official banks were founded. Therefore, it was expected that interlocking directorate networks had arisen precisely in that decade. Actually, Santiago Roldán and José Luis García Delgado's work established the existence of this network in 1921 and Juan Muñoz's book verified its persistence in 1966-67..$^{5}$ Consequently, the long-run collusive hypothesis would become proved, lasting from the 1920s until the 1960s.

Recently, the traditional hypothesis has been challenged. It has been noted that economic concentration was not in every moment as high as it was presumed, nor it stood out in an international comparison. ${ }^{6}$ Existence of collusive agreements has been verified, but it has also realized their short length and their negligent implementation by signatories. ${ }^{7}$ Likewise, the seemingly restrictive status quo regulation did not always represent any bar for rivalry among financial firms, because government usually

\footnotetext{
${ }^{4}$ See De la Sierra (1951), Muñoz Linares (1952, 1955), Tamames (1961), and Muñoz (1969).

${ }^{5}$ See Roldán-García Delgado (1973), vol. 2, pp. 264-269, and Muñoz (1969), ch. 4.

${ }^{6}$ See Fanjul-Maravall $(1982,1985)$ and Pueyo (2003).

${ }^{7}$ See García Ruiz (2002) and García Ruiz-Tortella (2003).
} 
enforced laws in a discretionary way. ${ }^{8}$ Panorama raised by these researches indicates that the Spanish banking industry in the twentieth century was a typical oligopoly, because a few large banks intermediated most of the available loanable funds. However, it has to be stressed the presence of a large number of medium-size and small banks, some of them with well-established branch networks, and also of many saving banks that were gradually acting as real banking firms and giving up their charitable activities as time went on. ${ }^{9}$ Therefore, this large number of entities and their very asymmetric characteristics would hamper collusion. Besides, product differentiation and informational problems that characterize banking business would constitute additional handicaps for collusive behaviour.

The fact that Spanish banking industry was structured as an oligopoly since at least the First World War does not mean that it was necessarily a cartel or was exposed to permanent collusion. Obviously, Spanish banks had a strong incentive to collude and restrain competition: to share out monopolistic rents yielded from a cartelised market. But, as usual in this type of market structure, each firm would face the incentive of additional profits by cheating. Because of product differentiation and informational problems, it would be extremely difficult to monitor and enforce collusive agreements. Then, government support and its compulsory powers would have been needed to reinforce collusion, as it happened in other countries. ${ }^{10}$ However, knowledge about government banking policies before the civil war is deficient and, apparently, Franco's governments did not show excessive zeal to carry out with its part beyond a very keen interest in collecting funds to finance governmental budgets. ${ }^{11}$

On the other hand, literature about firm governance also casts doubts on the capabilities of interlocking directorates as coordinative agents. Interlocking directorate technique of analysis is based on the assumption that boards of directors' members hold a real competence to affect firm's performance. Hence, those seated in the boards of various firms in the same industry would get ready to coordinate firms' actions or convey significant information for this purpose. In fact, the collusive hypothesis in Spanish banking historiography is based explicitly on a Marxist approach, as is clear from Juan Muñoz's book quotes. Accordingly, capitalist economies would tend to concentrate economic power in hands of a social oligarchy, whose high representatives would be large bankers. Members of this oligarchy would seat in the boards of directors of large banks and firms, with the aim of coordinating their activities and restricting market competition, so the whole of the capitalistic economies would become monopolised. $^{12}$

However, current literature is sceptical in this regard. It considers that firm governance falls on executive managers, because they are who have real control on resources and information within the firm. Thus, boards of directors' capabilities would be very constrained because their main sources of information are precisely those executive managers whose performance is supposed they have to monitor in shareholders' behalf. It has even said that board membership would be useful for managers' prestige for the most part, because firm's reputation would enhance according to its directors' social or economic status. Other less extreme approach

\footnotetext{
${ }^{8}$ See Pons $(1999,2001,2002)$ and Faus (2001).

${ }^{9}$ See papers presented in session B8 of VIII AEHE Congress devoted to saving banks.

10 The British case is the best-known example of agreements between government and big banks to reinforce collusive pacts: see Capie-Billings (2004).

11 According to Poveda (1980), the main interest of Franco's governments with respect banking industry was to employ its deposits to finance public expenses, once sability was guaranteed by status quo regulation.

12 The origin of this theory is in Hilferding (1985).
} 
considers that interlocking directorates is one of the many mechanisms at firm's disposal in order to optimize its behaviour, that might include collusion or whatever other firms' target. ${ }^{13}$

In economic history, analysis of interlocking directorates has been applied in studies about the role of universal banks in the industrialisation of some Europeans countries. Common directors would be used to establish long-run relations between industrial enterprises and banks, which would retain hegemony. However, recent papers have challenged this hypothesis because of lack of adequate empirical evidence. These scholars conclude that board of directors' role is not identical in all firms and reasons for interlocking directorates could be very diverse, so no general conclusion should be held without a detailed study in each case. ${ }^{14}$

Consequently, there are a number of possible interpretations of inter-firm relationships by interlocking directorates. It could be an irrelevant link, a firm's devise to achieve some of its targets or an essential scheme for collusion. Therefore, interlocking directorate analysis depends critically on the directors' role in firm governance. If they played a symbolic role, giving up firm governance on executive managers, interlocking directors would be irrelevant and their analysis would be misleading. Alternatively, executive managers may use directors to attain firm's targets, whatever they could be. In this case, interlocking directors might reflect entrepreneurial strategies, which should be the main consideration in research. In this context, interlocking directorate analysis may be used as a first step to select a group of apparently connected firms.

\section{Interlocking directorates in Spanish banking in the twentieth century.}

Despite lack of accuracy and insufficient empirical evidence, bank coordination by interlocking directorates has been accepted within Spanish historiography as a correct explanation of contemporary banking history. In this section, new empirical evidence about interlocking directorates in Spanish banking in the twentieth century is displayed. ${ }^{15}$ Our aim is to check whether more systematic evidence established by interlocking directorate analysis is consistent with current conclusions. Thus, it has been recreated bank networks according to the method and sources used by quoted authors, but for a larger number of years to cover the whole twentieth century.

Early works have been our starting point. ${ }^{16}$ They established networks of interlocking directorates for the years 1921, 1961 and 1966-67. For our research, additional years were selected: previous (1915), in-between (1925, 1934 and 1946) and subsequent $(1975,1980,1985$ y 1990) to former dates. Sources are the same employed

\footnotetext{
13 There are no definitive conclusions about the role of directors and their influence on managers and, therefore, firms' strategies and performance: see Hermalin-Weisbach (2003). For instance, Mace (1986) presents some directors and managers' statements to conclude that board of directors are irrelevant in the firm's desicion process; Termes (1991) denies that board of directors took part in Spanish banks' governance.

${ }^{14}$ See Fohlin (1999) and Tomka (2001). Guinnane (2002) sets German universal banks in the context of the whole financial sector and its historical evolution, as a consequence he undermines the role of universal banks in the process of industrialization.

${ }^{15}$ Bank connections and resultant clusters are showed in appendixes at the end of this paper as well as the complete lists of interlocking directors.

${ }^{16}$ See Roldán and García Delgado (1973), vol. 2, pp. 264-269, and Muñoz (1969), ch. 4. Other works in the same vein are: De la Sierra (1951) with data for the year 1949, Tamames (1961) for the year 1958, second edition of this same reference (1966) for the year 1963 and Tamames (1977) for 1975.
} 
by quoted scholars: Anuario Financiero y de Valores Mobiliarios, year I (1916) to establish banking directors' membership in 1915 and Anuarios Financieros y de Sociedades Anónimas years 1926, 1935 and 1947 for directories in 1925, 1934 and 1946, respectively. Unfortunately, these Anuarios did not cover the whole banking industry. Editors only published information of those banks that decided to send it voluntarily. Even though, Anuarios include information about all large banks and most of the medium-size and small entities. For 1975, 1980, 1985 and 1990, our sources has been Anuarios Estadísticos de la Banca Privada, published by Asociación Española de Banca since the 1970s, that collect information of the whole banking industry.

Initial year was set in 1915 in order to detect banking groups before the full impact of First World War on the Spanish economy. Then, years 1925 and 1934 were considered to check whether banking networks detected by Roldán and García Delgado in 1921 overcame interwar political and economic turmoil. 1946 was selected as the representative year of the post-civil war, when banking activity was already normalized and status quo regulation was just to be reinforced with the enactment of a new Banking Act. Comparison with pre-war groups would allow us to take account of civil war and post-war consequences. Then, networks detected by Juan Muñoz in 1961 and 1966-67 come to the fore, that would be affected by status quo regulation and Autarky. Comparison with networks from 1975 on would permit us to verify the validity of our hypothetical banking cartel as well as its progressive dissolution if this had been the case.

Owing to the fact that our working hypothesis holds that main actors in collusion were large banks, then associations or coalitions of banking firms have been classified under the name of the correspondent large bank. This classification does not prejudge that those banks were in fact the leaders. It has to be considered as a devise to put some order in the information given and, besides, it is in accordance with our working hypothesis. Spanish large banks were Hispano Americano, Español de Crédito (Banesto), Bilbao, Vizcaya and Central for the whole period. Banco de Santander climbed to this category in the 1950s (here, it appears since 1961's coalitions) and Banco Popular rises in the 1960s (since 1966-67's associations). Links among Catalan banks are also showed for the period before the civil war because they seemed to play an important role in large Spanish banks relationships. Appendix 1 shows the lists of banks in each association or group and appendix 2 displays corresponding diagrams. ${ }^{17}$ Two types of connections are displayed: banks with multiple links among them and other entities connected by just one director; this are entitled as "others" in italics.

It is important to bear in mind the rule used to demarcate bank associations. Only interlocking directorates have been taken into account, regardless of any other relations in terms of proprietorship or explicit agreements. A review of current literature about Spanish bank history would give sense to some of these relations and, probably, dismiss others.

\section{Bank networks in Spanish banking in the twentieth century.}

In this section, results are examined chronologically to check the hypothesis of permanent collusion in Spanish banking in the twentieth century. Appendix 3 displays diagrams of connections between groups, standing out bridge banks if appropriate. In 1915 , the first year of our database, it can hardly be considered that there were banking

\footnotetext{
${ }^{17}$ Appendix 4 presents complete lists of interlocking directorates.
} 
groups. We only have the pair formed by Banco de Bilbao and Banco del Comercio, and a trio around Banco Hispano Americano. Some weak relations among Catalan banks have to be added, but there is only one director between each pair of entities. It cannot be accepted that a network of interlocking directors connected the whole Spanish banking firms before First World War.

The state of play becomes more intricate in the 1920s, because of a large number of links among banks. This is the network detected in Santiago Roldán and José Luis García Delgado's research. However, two large banks, Banco Hispano Americano and Banco de Vizcaya, had no connection with any other bank. The case of Hispano Americano is especially significant because this bank had occupied the second position in the ranking of deposits since 1909, after Banco de España, and arrived at the first position in 1929. In 1921, five top entities in terms of deposits were in the web: Banesto (3rd), Urquijo (4th), Bilbao (5th), Central (6th) and Crédito de la Unión Minera (8th). In 1925, density of connections remained, but two groups around Banco Urquijo and Banesto stood out from other coalitions. It is also apparent the increasing intricacy of connections among Catalan banks, which seem to gather around three or four entities: Banca Arnús, S.A. Arnús Garí or Banca Marsans and, finally, Banco de Cataluña. At the same time, Sindicato de Banqueros, Banco Internacional de la Industria y el Comercio, and Banco Hispano Colonial worked as links between these groups. State of affairs, seemingly, was marked by a huge complexity, with crossrelations among most of the largest banks of the period. ${ }^{18}$ However, this situation can be clarified gathering each coalition under the name of the associated large bank (see diagrams in appendix 3). Then, network appearance disappears and only subsists for the Catalan banks. Large banks from Madrid and Bilbao appear weakly linked, only by one director and, sometimes, with a small bank between them. Catalan banks apart, the network of interlocking directorates seems very poor.

In 1934 we can see that most of the links of the 1920s have disappeared. The general network connected only Banco Urquijo's group with Banco Central's coalition through a pair of Catalan banks. Other associations appear clearly divided from each other, Banesto had consolidated its associates and only a web of Catalan banks persisted, with Banco Central embedded into it.

According to collusive hypothesis, the increase in the number of connections during the First World War years and the 1920s can be explained as a result of a process of general collusion that started during the war years and was promoted by the 1921 banking act. However, consequently with this hypothesis, we have to conclude that cartelization failed between 1925 and 1934 or, at least, there was a radical change in the strategy adopted to control financial markets, because most of the bank connections disappeared. Collusive hypothesis supporters, however, have not considered any rearrangement of this kind.

Immediately after the civil war, there was an extremely confusing period because of monetary reunification process and clearing of banking operations carried out during the war. Banking operations resumed from 1942 onward and government took advantage of bringing Bank of Spain's note issue monopoly up to date to revise the banking law and consolidate status quo regulation introduced during the civil war and post-war years. In this context, in 1946, it can be seen that large banks maintained a substantial number of interlocking directorates with other banks, but there was no network linking the whole of the big banks. It was just Banesto and Banco de Bilbao that appeared in relation with each other, but with two intervening entities between

\footnotetext{
${ }^{18}$ See graph in Roldán-García Delgado (1973), vol. 2, p. 267.
} 
them. In the middle of Franco's Autarky, which has been considered the age of gold for large Spanish banks, we find well-delimited groups around large entities but without connections among them. There is no network connecting the whole banking system, as collusive hypothesis would predict. It does not seem that status quo regulation drove Spanish banks inevitably into cartelization or, at least, interlocking directorates do not provide any sign of this hypothetical collusion.

In the 1960s, it can be observed a process bearing strong resemblance to that occurred in the interwar years. Connections between large banks' groups increased in number and, in 1966-67, a dense web appears clearly. However, this network disappeared again in 1975. Therefore, as it had happened in the years prior to the civil war, if large banks had tried to use a web of interlocking directors to support any hypothetical collusion, then after 1967 there would be a change in strategy or, alternatively, collusion would fail.

In 1980, a network of interlocking directorates was rebuilt another time but, looking at the corresponding diagram, it is realized that large banks are located in the fringes of the web. Notice that firms connecting large banks were very peculiar: two banks of mixed owner capital (foreign and national) and two groups of banks controlled by financial authorities. As it is known, financial authorities sorted out Spanish banking crisis in collaboration with private banks. Therefore, only a few banks went into liquidation, because most of the banks in distress were taken over by other private entities as part of a process of reorganization. These were the origin of connections detected in the 1980s. ${ }^{19}$

When the banking crisis was over and the Spanish economy was integrated in the European Communities, there were a series of acquisitions and consolidations that shaped the current banking groups. Now, a large universal bank is associated with a set of specialized entities (private banking, e-banking, etc). No interlocking director connected large banks' groups in 1990, except an indirect link between Banesto and BBV through Banco Saudí Español.

\section{Conclusions}

Some scholars suggested that Spanish banks acted as a monopoly since the First World War until the last decades of the twentieth century. Main actors would have been the 5-7 large banks, but "actual state of banking system can only be analysed properly when we take into account banking groups heading by the largest entities". ${ }^{20}$ According to this hypothesis, banking groups were delimited indirectly by a type of connections that were considered of high significance: interlocking directorates, family bonds, and institutional relationships (CSB, official banks). It was reached the conclusion that a very dense web of interlocking directorates connected all large banks and part of the medium-sized and small banks. By these means big banks would coordinate their actions aimed to a mutual target: maximization of joint profits by cartelization of financial markets. Besides, it was said that this network persisted without a break at least since the 1920s until the end of the 1960s. In this paper this type of analysis has been replied in a systematic way. Banking associationss have been delineated according to interlocking directorates method covering the whole twentieth century. The aim is to check the validity of the collusive hypothesis or whether networking resulted from peculiar circumstances of each period.

\footnotetext{
${ }^{19}$ About Spanish banking crisis, see Cuervo (1988).

${ }^{20}$ Muñoz (1969), p.103.
} 
Our results show that history of inter-bank relationships appears more complex than it was supposed. Interestingly, links among large banks were not long-lived, so it should be rejected that interlocking directorates worked as a long-lasting coordination scheme of a hypothetical banking cartel. Certainly, these networks were especially dense in 1925 and 1966-67, but they disappeared quickly, so a few years later we find that each large bank maintained its own group, distinctly apart from the others.

On the eve of the First World War, large banks held only a few connections to other entities. It was in 1921-25 when a network of interlocking directorates became apparent. Yet, oligopolistic coordination is not the sole feasible explanation, particularly if it is noted that two large banks, including the leader in deposits, kept out of the web. Other interpretations are possible and a detailed survey of bank histories may suggest additional hypothesis for further research. But, even though actual network would have been a sign of collusion, then this conduct would fail between 1925 and 1934 or, alternatively, coordination schemes would have experienced a radical change, that would deserve some explanation.

After the civil war, no interlocking directorates network appeared before the 1960s but, as it happened before the civil war, the web disappeared within a few years later. A process similar to that occurred in the 1920s seems to have taken place in the 1960s, so that future research will have to take this resemblance into account. However, now it is clear that regulation is not a direct cause of the emergence of this type of bank relationship. Finally, connections detected between 1980 and 1990 would be a byproduct of the banking crisis. Large banks took over most of the ruined banks as part of a plan agreed with public authorities. Other connections, increasingly large as the end of the century drew closer, were caused by a new banking strategy consisting of a holding of a large banks associated with specialized financial firms.

\section{Bibliography}

AA.VV. (1954), Notas sobre política económica española, Madrid, Publicaciones de la Delegación Nacional de FET y de las JONS.

CAPIE, Forrest and Mark BILLINGS (2004), "Evidence on competition in English commercial banking, 1920-70", Financial History Review, 11(1):69-103.

CUERVO, Álvaro (1988), La crisis bancaria en España, 1977-1985. Causas, sistemas de tratamiento y coste, Barcelona, Ariel.

DE LA SIERRA, Fermín (1951): "La situación monopolística de la banca privada en España", Revista de Economía Política, vol. 3, nº 1-2.

FANJUL, Oscar y Fernando MARAVALL (1982), "Estructura de mercado y crecimiento del sistema bancario español: un análisis de las tres últimas décadas", Cuadernos Económicos de ICE, num. 21.

FANJUL, Oscar y Fernando MARAVALL (1985), La eficiencia del sistema bancario español, Madrid, Alianza.

FAUS, E.M. (2001), Regulación y desregulación. Notas para la historia de la banca española, Barcelona, Península.

FOHLIN, Caroline (1999): "The Rise of Interlocking Directorates in Imperial Germany", Economic History Review, 52(2):307-333.

GARCÍA RUIZ, J.L. (2002), "Los arreglos interbancarios durante el franquismo", Revista de Historia Económica, año XX, $\mathrm{n}^{\circ} 2$. 
GARCÍA RUIZ, J.L., G. TORTELLA (2003), "Banca y política durante el primer franquismo", en G. Sánchez Recio, J. Tascón, eds., Los empresarios de Franco. Política y economía en España, 1936-1957, Barcelona, Crítica.

GUINNANE, Timothy W. (2002), "Delegated monitors, large and small: Germany's banking system, 1800-1914", Journal of Economic Literature, 40(1):73-124.

HERMALIN, Benjamin, WEISBACH, Michael (2003): "Boards of directors as an endogenously determined institution: A survey of the economic literature", Federal Reserve Bank of New York Economic Policy Review, april, p.7-26.

HILFERDING, Rudolf (1985): El capital financiero, Madrid, Tecnos. Edición original de 1910

MACE, M. (1986): Directors: Myth and Reality, Boston, Harvard Business School Press.

MUÑOZ, Juan (1969): El poder de la banca en España, Madrid, ZYX.

MUÑOZ LINARES, Carlos (1952): "La concentración de capital en hs sociedades y empresas españolas", Revista de Economía Política, vol. 4, n 1.

? (1955): "El pliopolio en algunos sectores del sistema económico español", Revista de Economía Política, vol. 7, n ${ }^{\circ} 1$.

PONS, M ${ }^{\mathrm{a}}$ A. (1999), "Capture or agreement? Why Spanish banking was regulated under the Franco regime (1939-1975)", Financial History Review, 6(1).

PONS, M A A. (2001), "Oligopolio y tipos de interés en la banca española, 1942-1975", Revista de Historia Económica, año XIX, nº 3, pg. 679-703.

PONS, M ${ }^{\mathrm{a}}$ A. (2002), Regulating Spanish banking, 1939-1975, Ashgate, Aldershot.

POVEDA, Raimundo (1980), "Política monetaria y financiera", en L. Gámir, ed., Política económica de España, vol. 1, Madrid, Alianza, $3^{\mathrm{a}}$ ed.

PUEYO, Javier (2003), "Oligopolio y competencia en la banca española del siglo XX", Revista de Historia Económica, año XXI, $\mathrm{n}^{\circ} 1$.

PUEYO, Javier (2005), Inter-firm relationships and interlocking directorates in Spanish banking in the twentieth century, ¿Investigaciones de Historia Económica?

PUEYO, Javier (forthcoming), La gran banca en España, 1921-1974: colusión, regulación y rivalidad, Banco de España, Servicio de Estudios, Estudios de Historia Económica.

ROLDÁN, Santiago, GARCÍA DELGADO, José Luis, MUÑOZ, Juan (1973): La formación de la sociedad capitalista en España, 1914-1920, Madrid, CECA.

TAMAMES, Ramón (1961): La lucha contra los monopolios, Madrid, Tecnos.

TAMAMES, Ramón (1977): La oligarquía financiera en España, Madrid.

TERMES, R. (1991): Desde la banca, 2 vol., Madrid, Rialp.

TOMKA, B. (2001): "Interlocking Directorates between banks and Industrial Companies in Hungary at the Beginning of the Twentieth Century", Business History, 43(1):25-42.

TORTELLA, Gabriel (2000), The Development of Modern Spain: An Economic History of the Nineteenth and Twentieth Centuries, Cambridge, Harvard University Press. 


\section{Appendix 1. Associations of banks}

Associations or groups of banks are delimited exclusively on the basis of interlocking directorates among private banks. No other type of relationship has been taken into account. Particularly, neither property of shares nor explicit agreements between banks was brought into play for two reasons. Firstly, because this information is currently unknown, but mainly because we are not interested in delimiting actual banking groups but in checking an explicit hypothesis about the relevance of interlocking directorship.

Groups are organized according to the associated largest bank. This classification does not take for granted that large banks exerted leadership or there was any type of joint strategy whitin each coalition. However, this is the most suitable procedure to organize information because it fits accurately our working hypothesis. That is to say, large banks would play the leading role in the functioning of the banking system and interlocking directorates would be one the main schemes of control and coordination. The same five banks held top positions in bank rankings during the whole period: Banco Hispano Americano, Banco Español de Crédito (Banesto), Banco de Bilbao, Banco de Vizcaya and Banco Central. Banco de Santander entered into this category in the 1950s (it appears here since 1961 diagrams) and Banco Popular appears in the 1960s (since 1966-67 diagrams). Connections among Catalan banks before the civil war are also showed because they played an important role in order to explain links between large Spanish banks's groups.

Two sets of banks are singled out within each cluster. A first set is composed of those entities with multiple links among then. These are reckoned as the genuine members of each banking group. Second, banks connected with the previous ones by only one director are displayed apart (under the rubric "others" in the lists and into an ellipsis in the diagrams) and have not been considered as members of the group properly.

Associations of 1921, 1961 and 1966-67 were drawn from secondary sources: Roldán and García Delgado (1973), and Muñoz (1969). Those of 1915, 1925, 1934 and 1946 have been built crossing lists of banking directors collected from Anuario Financiero y de Valores Mobiliarios, year 1916, and Anuarios Financieros y de Sociedades Anónimas, years 1926, 1935 and 1947, respectively. Unfortunately, Anuarios do not have universal coverage because they published only information sent voluntarily by firms. However, directorates of all large banks and most of the mediumsize and small joint-stock banks are available in the source. Next, it comes the list of banks whose directorates were collected:

\section{Banks}

B. Agrario de Baleares

B. Agrícola Andaluz

B. Albacete

B. Ampurdán

B. Aragón

B. Aragonés de Crédito

B. Asturiano de Industria y Comercio

B. Ávila

B. Badalona

$\begin{array}{cccc}1915 & 1925 & 1934 & 1947 \\ \text { X } & \text { X } & & \text { X } \\ & \text { X } & & \\ \text { X } & & & \\ \text { X } & & & \\ \text { X } & \text { X } & \text { X } & \text { X } \\ \text { X } & & \text { X } & \text { X } \\ \text { X } & \text { X } & \text { X } & \text { X } \\ & \text { X } & \text { X } & \\ & \text { X } & \text { X } & \text { X }\end{array}$


B. Barcelona X

B. Bilbao

B. Burgos

B. Calamarte

B. Cartagena

B. Castellano

B. Castellón

B. Castilla

B. Cataluña

B. Central

B. Coca

B. Comarcal de Crédito

B. Comercial de Barcelona

B. Comercial de Ciudadela

B. Comercial de Tarragona

B. Comercial de Tarrasa

B. Comercial Español

B. Comercio

B. Crédito Ampurdanés

B. Crédito de Zaragoza

B. Crédito Industrial

B. Crédito Local de España

B. Elda

B. España

B. Español de Crédito

B. Español del Rio de la Plata

B. Español en París

B. Exterior de España

B. Felanitx

B. Ferrerías

B. Garriga Nogués

B. General de Administración

B. Germánico de América del Sur

B. Gijón

B. Gijonés de Crédito

B. Granollers

B. Guipuzcoano

B. Herrero

B. Hipotecario

B. Hispanense

B. Hispano Americano

B. Hispano Colonial

B. Hispano Suizo

B. Ibérico

B. Internacional de Industria y Comercio

B. Irún

B. Jerez

B. La Coruña

B. Manacor

B. Manchego

B. Menorca

B. Mercantil

B. Mercantil Agrícola

B. Mercantil de Menorca

B. Mercantil de Tarragona
X

$X \quad X$

$\mathrm{X}$

$\mathrm{X}$

X $\quad$ X $\quad$ X $\quad$ X

X X

X

$\mathrm{X}$

X $\quad$ X $\quad$ X

X $\mathrm{X}$

$\mathrm{X}$
$\mathrm{X}$

$\mathrm{X} \quad \mathrm{X}$

$\mathrm{X}$

X X X

$\mathrm{X}$

$\begin{array}{llll}X & X & X & X\end{array}$

$X$

$\begin{array}{lll}X & X & X\end{array}$

X X

X $\mathrm{X}$

X $X$

$\begin{array}{llll} & X & X & \\ X & X & X & X\end{array}$

$\mathrm{X}$

X

$\begin{array}{cccc} & & X & X \\ & X & X & \\ & X & X & X \\ & X & & X \\ & & & X\end{array}$

$\begin{array}{llll} & X & X & X \\ X & X & X & X\end{array}$

$\mathrm{X}$

$\begin{array}{llll} & & X & \\ X & X & X & X\end{array}$

$\begin{array}{llll}X & X & X & X\end{array}$

X X X

$\begin{array}{llll} & X & & \\ X & X & X & X\end{array}$

X $\quad$ X $\quad$ X $\quad$ X

X $X \quad X$

$\begin{array}{lll}X & X & \\ & X & X\end{array}$

$\begin{array}{ll} & X \\ X & X\end{array}$

$\begin{array}{lll}X & X & X \\ & X & X\end{array}$

$\begin{array}{llll} & X & & \\ X & X & X & \\ X & X & X & X \\ & X & & \end{array}$

X X 
B. Mercantil e Industrial

B. Minero Industrial de Asturias

B. Oeste de España

B. Oviedo

B. Palafrugell

B. Pastor

B. Penedés

B. Popular de Crédito y Descuento

B. Popular de Manacor

B. Popular Español

B. Préstamos y Descuentos

B. Propiedad

B. Reus

B. Rural

B. Sabadell

B. Sainz

B. San Sebastián

B. Santander

B. Sóller

B. Tarrasa

B. Tolosa

B. Torrelavega

B. Tortosa

B. Úbeda

B. Urquijo

B. Urquijo Catalán

B. Urquijo de Guipúzcoa

B. Urquijo Vascongado

B. Valencia

B. Valls

B. Valores y Crédito

B. Vigo

B. Villanueva

B. Viñas-Aranda

B. Vitoria

B. Vizcaya

B. Zaragozano

Banca Arnús

Banca Díez Vergara

Banca López Bru

Banca López Quesada

Banca March

Banca Marsans

Banca Mir

Banca Nonell

Banca Rosés

Banca Suñer

Banca Tusquets

Clemente Sánchez

Crédito Balear

Crédito de la Unión Minera

Crédito Industrial y Mercantil

Crédito Mercantil

Crédito Mercantil de Menorca

Crédito Navarro

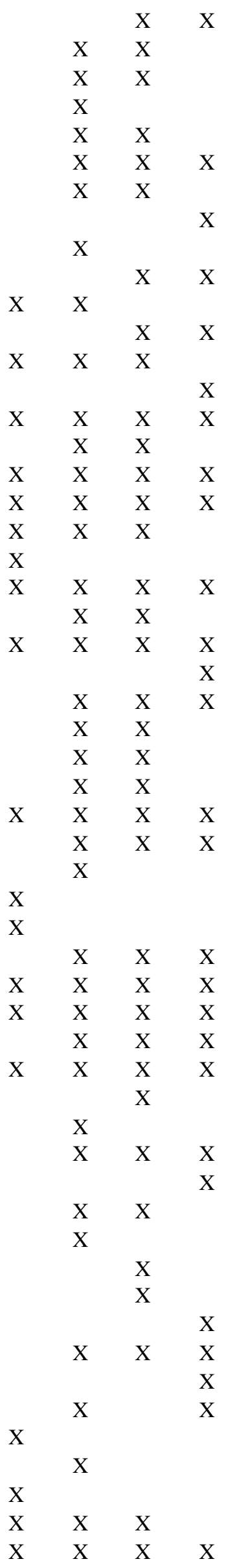


Crédito y Docks de Barcelona

Crédito y Fomento de Ahorros

Epifanio Ridruejo

Fomento Agrícola de Mallorca

Fomento Agrícola Industrial y Comercial

Jáudenes Bárcena

La Agrícola (sección de banca)

La Puebla, S.A.

La Vasconia

Mariano Borrero Blanco

Martínez Montiel

Matías Blanco Cobaleda

Pérez López

S. A. Arnús i Garí

S. de Créditos Hipotecarios y Personales

Sindicato de Banqueros

$\begin{array}{llll}\text { X } & \text { X } & \text { X } & \text { X } \\ & \text { X } & \text { X } & \text { X } \\ & & & \text { X } \\ \text { X } & \text { X } & \text { X } & \text { X } \\ \text { X } & \text { X } & \text { X } & \\ & & & \text { X }\end{array}$

Númber of banks

X

$X \quad X$

$\begin{array}{llll}X & X & X & X\end{array}$

$x$

$\mathrm{X}$

$X$

$\mathrm{X}$

$\begin{array}{lll}X & X & X\end{array}$

$\begin{array}{llll} & & X & \\ X & X & X & X\end{array}$

$\begin{array}{llll}50 & 91 & 86 & 70\end{array}$

Associations of 1975, 1980, 1985 and 1990 were built from directorates of all Spanish private banks, picked up from Anuarios Estadísticos de la Banca Privada, years 1975, 1980, 1985, and 1990, that offer universal coverage. For the last year, 1990, the source includes the lists of current banking groups' members, according to head banks' Annual Reports. An asterisk marks actual members of each banking group in our lists and diagrams. 
Table 1. Banco Hispano Americano

\begin{tabular}{|c|c|c|c|}
\hline 1915 & 1921 & 1925 & 1934 \\
\hline $\begin{array}{c}\text { Herrero } \\
\text { Gijón }\end{array}$ & $\begin{array}{c}\text { Herrero } \\
\text { Gijón } \\
\text { San Sebastián } \\
\end{array}$ & $\begin{array}{c}\text { Herrero } \\
\text { Gijón } \\
\text { San Sebastián }\end{array}$ & $\begin{array}{c}\text { Herrero } \\
\text { Gijón } \\
\text { San Sebastián }\end{array}$ \\
\hline 1947 & 1961 & $1966-67$ & 1975 \\
\hline $\begin{array}{c}\text { Herrero } \\
\text { Gijón } \\
\text { San Sebastián } \\
\text { Urquijo } \\
\text { Mercantil Tarragona } \\
\text { Valls } \\
\text { Propiedad } \\
\text { Zaragozano }\end{array}$ & \begin{tabular}{|l|}
\multicolumn{1}{|c|}{ Herrero } \\
\multicolumn{1}{|c|}{ Gijón } \\
\multicolumn{1}{|c|}{ San Sebastián } \\
$\quad$ Urquijo \\
\\
Others: \\
Tolosa (see Bilbao) \\
Mercantil Tarragona
\end{tabular} & \begin{tabular}{|l}
\multicolumn{1}{|c}{ Herrero } \\
\multicolumn{1}{|c}{ Gijón } \\
\multicolumn{1}{|c}{ San Sebastián } \\
$\quad$ Urquijo \\
Mercantil Tarragona \\
\multicolumn{1}{c}{ Valls } \\
Others: \\
Tolosa (see Bilbao) \\
Aragón (see Central)
\end{tabular} & $\begin{array}{l}\text { Others: } \\
\text { Gijón }\end{array}$ \\
\hline 1980 & 1985 & $1990\left(^{*}\right)$ & \\
\hline \begin{tabular}{l}
\multicolumn{1}{c}{ Urquijo } \\
Hispa.Ind. (ex-Valls) \\
Mercantil Tarragona \\
Others: \\
Árabe Español
\end{tabular} & \begin{tabular}{l}
\multicolumn{1}{|c|}{ Urquijo-Unión } \\
Hispano Industrial \\
Mercantil Tarragona \\
$\quad$ Jerez \\
$\quad$ Norte \\
Others: \\
Saudí Español (see \\
Banesto and Vizcaya)
\end{tabular} & $\begin{array}{l}\text { Others: } \\
\text { Jerez } \\
\text { Banif (ex-Norte) }\end{array}$ & \\
\hline
\end{tabular}

(*) According to Anuario Estadístico de la Banca Privada, year 1990, Banco Hispano Americano group comprised Banif, Banco de Jerez and Banco Mercantil de Tarragona.

Table 2. Banco Urquijo (*)

\begin{tabular}{|c|c|c|c|}
\hline 1915 & 1921 & 1925 & 1934 \\
\hline & $\begin{array}{l}\text { Urquijo Catalán } \\
\text { Urquijo Vascongado } \\
\text { Urquijo Guipúzcoa } \\
\text { Minero Ind. Asturias } \\
\text { Valencia } \\
\text { Asturiano } \\
\text { Others: } \\
\text { Banesto } \\
\text { Gijonés de Créd. } \\
\text { Hispano Colonial }\end{array}$ & \begin{tabular}{l}
\multicolumn{1}{c}{ Urquijo Catalán } \\
Urquijo Vascongado \\
Urquijo Guipúzcoa \\
Minero Ind. Asturias \\
$\quad$ Valencia \\
$\quad$ Asturiano \\
\\
Others: \\
Banesto-Gijonés de \\
Créd.-Com. Español \\
Hispano Colonial \\
Burgos \\
Bilbao
\end{tabular} & \begin{tabular}{l} 
Urquijo Catalán \\
Urquijo Vascongado \\
Urquijo Guipúzcoa \\
Minero Ind. Asturias \\
Oeste de España \\
Mercantil Tarragona \\
\multicolumn{1}{c}{ Valls } \\
Others: \\
Hispano Colonial (see \\
Central) \\
Arnús \\
Créd. y Docks
\end{tabular} \\
\hline
\end{tabular}

(*) Since 1944, Banco Urquijo is included in Banco Hispano Americano's group. 
Table 3. Banco Español de Crédito

\begin{tabular}{|c|c|c|c|}
\hline 1915 & 1921 & 1925 & 1934 \\
\hline Hipotecario & $\begin{array}{l}\quad \text { Gijonés de Créd. } \\
\quad \text { Com. Español } \\
\text { Others: } \\
\text { Urq. Guipuzcoano } \\
\text { Castellano (see } \\
\text { Central) } \\
\text { Oviedo }\end{array}$ & $\begin{array}{l}\text { Gijonés de Créd. } \\
\text { Com. Español } \\
\quad \text { Oviedo } \\
\text { Others: } \\
\text { Urq. Guipuzcoano }\end{array}$ & \\
\hline 1947 & 1961 & 1966-67 & 1975 \\
\hline $\begin{array}{l}\quad \begin{array}{l}\text { Guipuzcoano } \\
\quad \text { Vitoria }\end{array} \\
\\
\\
\text { Others: } \\
\text { Pastor } \\
\text { Epifanio Ridruejo } \\
\text { Fom. Agric. Mallorca } \\
\text { (see Bilbao) }\end{array}$ & \begin{tabular}{l}
\multicolumn{1}{c}{ Guipuzcoano } \\
$\quad$ Vitoria \\
Epifanio Ridruejo \\
\\
Others: \\
Masaveu
\end{tabular} & 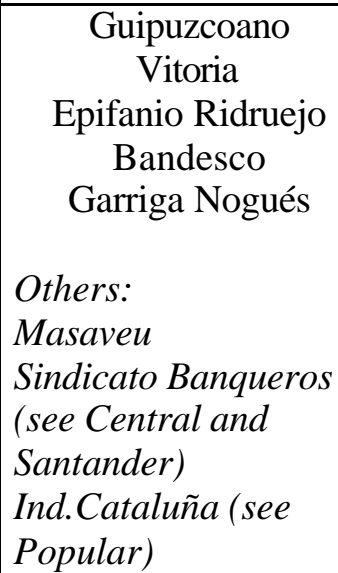 & $\begin{array}{c}\text { Guipuzcoano } \\
\text { Bandesco }\end{array}$ \\
\hline 1980 & 1985 & $1990(*)$ & \\
\hline $\begin{array}{l}\text { Guipuzcoano } \\
\text { Bandesco } \\
\text { Others: } \\
\text { Saudí Español }\end{array}$ & \begin{tabular}{l}
\multicolumn{1}{c}{ Guipuzcoano } \\
$\quad$ Bandesco \\
Garriga Nogués \\
$\quad$ Madrid \\
Catalán Desarrollo \\
Others: \\
Saudí Español (see \\
Hispano Americano \\
and Vizcaya) \\
Vitoria (see \\
Santander)
\end{tabular} & \begin{tabular}{l}
$\quad \begin{array}{c}\text { Bandesco } \\
\quad \text { Madrid }\end{array}$ \\
\multicolumn{1}{c}{ Català Crèd. (ex- } \\
Catalán Desarrollo) \\
\multicolumn{1}{c}{ General } \\
Others: \\
Vitoria \\
Saudí Español (see \\
BBV) \\
Garriga Nogués \\
Peninsular \\
Alicantino \\
Albacete
\end{tabular} & \\
\hline
\end{tabular}

(*) According to Anuario Estadístico de la Banca Privada, year 1990, Banesto group comprised Banco de Madrid, Banc Català de Crédit, Banco de Vitoria, Banco de Desarrollo Económico Español and Banco General. 
Table 4. Banco Central

\begin{tabular}{|c|c|c|c|}
\hline 1915 & 1921 & 1925 & 1934 \\
\hline & $\begin{array}{l}\text { Créd. Unión Minera } \\
\\
\text { Others: } \\
\text { Bilbao } \\
\text { Castellano (see } \\
\text { Banesto) } \\
\text { Santander } \\
\text { Cía. Créd. Bancario }\end{array}$ & $\begin{array}{l}\text { Castellano } \\
\text { Créd. Zaragoza } \\
\\
\text { Others: } \\
\text { Bilbao-Comercio }\end{array}$ & $\begin{array}{c}\text { Badalona } \\
\text { Hispano Colonial } \\
\quad \text { Arnús } \\
\quad \text { Créd. y Docks } \\
\quad \text { Menorca } \\
\text { Others: } \\
\text { Créd. Zaragoza } \\
\text { Urquijo Catalán } \\
\text { Propiedad } \\
\text { López Quesada }\end{array}$ \\
\hline 1947 & 1961 & 1966-67 & 1975 \\
\hline $\begin{array}{l}\text { Hispano Colonial } \\
\quad \text { Créd. y Docks } \\
\quad \text { Menorca } \\
\quad \text { Valencia } \\
\quad \text { Popular } \\
\text { Others: } \\
\text { Santander } \\
\text { Jerez } \\
\text { Tusquets } \\
\text { Sindicato Banqueros } \\
\text { Badalona }\end{array}$ & \begin{tabular}{l}
\multicolumn{1}{c}{ Créd. y Docks } \\
Valencia \\
\multicolumn{1}{c}{ Agric. Aragonés } \\
$\quad$ Popular \\
$\quad$ Andalucía \\
Salamanca \\
Others: \\
Sindicato Banqueros \\
(see Santander) \\
La Coruña
\end{tabular} & \begin{tabular}{l}
\multicolumn{1}{c}{ Créd. y Docks } \\
\multicolumn{1}{c}{ Valencia } \\
Agric. Aragonés \\
Aragón (v. Hispano) \\
\multicolumn{1}{c}{ Úbeda } \\
$\quad$ Fomento \\
Others: \\
Andalucía (see \\
Popular) \\
Sindicato Banqueros \\
(see Banesto, \\
Santander) \\
Atlántico \\
Exterior \\
Urquijo \\
Créd. Navarro \\
Ind. Cataluña (see \\
Banesto and Popular) \\
Catalán Desarrollo
\end{tabular} & $\begin{array}{l}\text { Valencia } \\
\text { Fomento }\end{array}$ \\
\hline 1980 & 1985 & $1990(*)$ & \\
\hline $\begin{array}{l}\text { Valencia } \\
\text { Fomento }\end{array}$ & 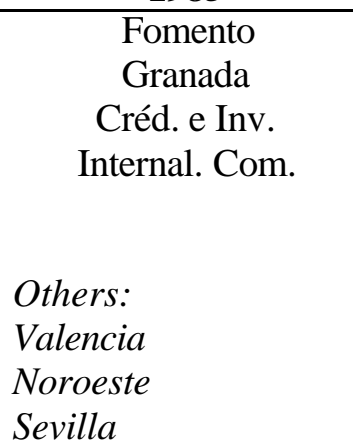 & $\begin{array}{l}\text { Valencia } \\
\text { Fomento } \\
\text { Granada } \\
\text { Gallego (ex-Créd. e } \\
\text { Inv.) } \\
\text { Internal. Com. } \\
\text { Others: } \\
\text { Valencia }\end{array}$ & \\
\hline
\end{tabular}

(*) According to Anuario Estadístico de la Banca Privada, year 1990, Banco Central group comprised Banco Internacional de Comercio, Banco Gallego, Banco de Granada, Banco de Fomento and Banco de Valencia. 
Table 5. Banco de Bilbao (*)

\begin{tabular}{|c|c|c|c|}
\hline 1915 & 1921 & 1925 & 1934 \\
\hline $\begin{array}{l}\text { Comercio } \\
\text { Others: } \\
\text { Cartagena }\end{array}$ & $\begin{array}{l}\quad \text { Comercio } \\
\text { Others: } \\
\text { Central } \\
\text { Sindicato Banqueros }\end{array}$ & $\begin{array}{l}\quad \text { Comercio } \\
\text { Others: } \\
\text { Asturiano (see } \\
\text { Urquijo) } \\
\text { Internal. Ind. y Com. } \\
\text { Central }\end{array}$ & $\begin{array}{l}\text { Comercio } \\
\text { Asturiano } \\
\text { Others: } \\
\text { Castellano }\end{array}$ \\
\hline 1947 & 1961 & 1966-67 & 1975 \\
\hline $\begin{array}{l}\quad \text { Comercio } \\
\quad \text { Asturiano } \\
\text { Fom.Agric.Mallorca } \\
\quad \text { (see Banesto) } \\
\text { Others: } \\
\text { Castellano } \\
\text { La Coruña } \\
\text { Tolosa }\end{array}$ & $\begin{array}{l}\quad \text { Comercio } \\
\quad \text { Asturiano } \\
\quad \text { Castellano } \\
\text { Others: } \\
\text { La Coruña (see } \\
\text { Central) } \\
\text { Tolosa (see Hispano) }\end{array}$ & \begin{tabular}{l}
\multicolumn{1}{c}{ Comercio } \\
\multicolumn{1}{c}{ Ind. de Bilbao } \\
$\quad$ Asturiano \\
$\quad$ Castellano \\
Others: \\
Coruña (see Popular) \\
Tolosa (see Hispano) \\
Irún \\
Málaga
\end{tabular} & $\begin{array}{c}\text { Comercio } \\
\text { Ind. de Bilbao }\end{array}$ \\
\hline 1980 & 1985 & $1990(\mathrm{BBV})(* *)$ & \\
\hline $\begin{array}{c}\text { Comercio } \\
\text { Ind. de Bilbao }\end{array}$ & $\begin{array}{l} \\
\text { Others: } \\
\text { Huesca } \\
\text { Extremadura } \\
\text { Promoción Negocios } \\
\text { Oeste } \\
\text { Sindicato Banqueros }\end{array}$ & $\begin{array}{l}\text { Comercio } \\
\text { Ind. de Bilbao } \\
\text { Banca Catalana } \\
\text { Ind. Cataluña } \\
\text { Ind. Mediterráneo } \\
\text { Barcelona } \\
\text { Occidental } \\
\text { Finanzia (ex-Oeste) } \\
\text { Créd. y Ahorro } \\
\text { Créd. Canario } \\
\text { Meridional } \\
\text { Privanza (ex-Ind. Sur) } \\
\text { Extremadura } \\
\text { Others: } \\
\text { Promoción Negocios } \\
\text { Saudí Español (see } \\
\text { Banesto) }\end{array}$ & \\
\hline
\end{tabular}

(*) Banco de Bilbao took over Banco de Comercio in 1901, although it was maintained as an independent entity. Diagrams from Roldán-García Delgado (1973), year 1921, does not display Banco de Comercio, but it has been included in this table.

(**) According to Anuario Estadístico de la Banca Privada, year 1990, Banco BilbaoVizcaya group comprised Banco de Comercio, Banco Industrial de Bilbao, Banca Catalana, Banco Industrial de Cataluña, Banco Industrial del Mediterráneo, Banco de Barcelona, Banco Occidental, Finanzia (ex-Banco del Oeste), Banco de Crédito y Ahorro, Banco de Crédito Canario, Banco Meridional, Privanza (ex-Banco Industrial del Sur), Banco de Extremadura, Banco de Promoción de Negocios and Bilbao Merchant Bank. 
Table 6. Banco de Vizcaya

\begin{tabular}{|c|c|c|c|}
\hline 1947 & 1961 & 1966-67 & 1975 \\
\hline & $\begin{array}{l}\text { Others: } \\
\text { Hispano Suizo } \\
\text { Vilella }\end{array}$ & & $\begin{array}{c}\text { Financiación Ind. (ex- } \\
\text { Hispano Suizo) }\end{array}$ \\
\hline 1980 & 1985 & 1990 & \\
\hline $\begin{array}{l}\text { Financiación Ind. } \\
\text { Others: } \\
\text { Créd. Com. } \\
\text { Saudí Español }\end{array}$ & $\begin{array}{l}\text { Financiación Ind. } \\
\text { Banca Catalana } \\
\text { Ind. Cataluña } \\
\text { Ind. Mediterráneo } \\
\text { Barcelona } \\
\text { Occidental } \\
\text { Créd. Com. } \\
\text { Créd. y Ahorro } \\
\text { Créd. Canario } \\
\text { Meridional } \\
\text { Ind. Sur } \\
\text { Others: } \\
\text { Saudí Español (see } \\
\text { Hispano and Banesto) } \\
\text { Huelva (see } \\
\text { Santander) } \\
\end{array}$ & (see B. Bilbao) & \\
\hline
\end{tabular}

Table 7. Catalan banks before the civil war: Banca Arnús

\begin{tabular}{|c|c|c|c|}
\hline 1915 & 1921 & 1925 & 1934 \\
\hline $\begin{array}{l}\text { Others: } \\
\text { Créd. y Docks } \\
\text { Sabadell }\end{array}$ & \begin{tabular}{l}
\multicolumn{1}{c}{ Créd. y Docks } \\
$\quad$ Menorca \\
$\quad$ Sabadell \\
Others: \\
Cía. Créd. Bancario \\
Sindicato Banqueros
\end{tabular} & $\begin{array}{l}\quad \begin{array}{l}\text { Créd. y Docks } \\
\quad \text { Menorca }\end{array} \\
\text { Others: } \\
\text { Sabadell } \\
\text { Com. Tarrasa (see } \\
\text { Marsans) }\end{array}$ & (see B. Central) \\
\hline
\end{tabular}

Table 8. Catalan banks before the civil war: Banco Hispano Colonial

\begin{tabular}{|l|l|l|l|}
\hline \multicolumn{1}{|c|}{$\mathbf{1 9 1 5}$} & \multicolumn{1}{|c|}{$\mathbf{1 9 2 1}$} & \multicolumn{1}{c|}{$\mathbf{1 9 2 5}$} & \multicolumn{1}{c|}{$\mathbf{1 9 3 4}$} \\
\hline \multirow{2}{*}{ Others: } & Others: & Others: & \\
Barcelona & Barcelona & Urquijo Catalán & \\
Arnús-Garí & Arnús-Garí & Mercantil & \\
Sindicato Banqueros & Cía. Créd. Bancario & López Bru & \\
& Urquijo Catalán & Internal. Ind.y Com. & \\
& & Arnús-Garí & \\
& & & \\
\hline
\end{tabular}


Table 9. Catalan banks before the civil war: Banco de Cataluña

\begin{tabular}{|l|l|l|l|}
\hline 1915 & \multicolumn{1}{|c|}{$\mathbf{1 9 2 1}$} & \multicolumn{1}{|c|}{$\mathbf{1 9 2 5}$} & 1934 \\
\hline & \multicolumn{1}{|c|}{ Reus } & \multicolumn{1}{|c|}{ Reus } & \\
& Tortosa & Tortosa & \\
& $\begin{array}{l}\text { Others: } \\
\text { Cía. Créd. Bancario }\end{array}$ & $\begin{array}{l}\text { Com. Tarragona } \\
\text { Sindicato Banqueros }\end{array}$ & \\
& $\begin{array}{l}\text { Sindicato Banqueros } \\
\text { Marsans }\end{array}$ & \\
\hline
\end{tabular}

Table 10. Catalan banks before the civil war: Banca Marsans

\begin{tabular}{|c|c|c|c|}
\hline 1915 & 1921 & 1925 & 1934 \\
\hline & $\begin{array}{l}\text { Others: } \\
\text { Tortosa (see } \\
\text { Cataluña) } \\
\text { Cía. Créd. Bancario } \\
\text { Sindicato Banqueros }\end{array}$ & $\begin{array}{l}\begin{array}{l}\text { Internacional Ind. y } \\
\quad \text { Com. } \\
\quad \text { Com. Tarrasa }\end{array} \\
\\
\text { Others: } \\
\text { Créd. y Docks (see } \\
\text { Arnús) } \\
\text { Prés. y Dtos.-Com. } \\
\text { Barcelona } \\
\text { Arnús-Garí } \\
\text { Hispano Colonial } \\
\text { Bilbao-Comercio } \\
\text { Sindicato Banqueros }\end{array}$ & $\begin{array}{l}\text { Internacional Ind. y } \\
\text { Com. } \\
\text { Com. Tarrasa } \\
\text { Com. Barcelona } \\
\text { Valencia } \\
\text { Castellón } \\
\text { Others: } \\
\text { Propiedad } \\
\text { Sindicato Banqueros }\end{array}$ \\
\hline
\end{tabular}


Table 11. Banco de Santander

\begin{tabular}{|c|c|c|c|}
\hline 1947 & 1961 & 1966-67 & 1975 \\
\hline & $\begin{array}{l}\quad \text { Soler y Torra } \\
\\
\text { Others: } \\
\text { Soler y Torra } \\
\text { Sindicato Banqueros } \\
\text { (see Central) }\end{array}$ & $\begin{array}{l}\text { Com. para América } \\
\text { (ex-Soler y Torra) } \\
\text { Intercontinental } \\
\text { Others: } \\
\text { Sindicato Banqueros } \\
\text { (see Banesto and } \\
\text { Central) }\end{array}$ & $\begin{array}{c}\text { Com. América } \\
\text { Bankinter } \\
\text { (ex-Intercontinental) }\end{array}$ \\
\hline 1980 & 1985 & $1990(*)$ & \\
\hline $\begin{array}{l}\text { Bank of America (ex- } \\
\text { Com. América) } \\
\quad \text { Bankinter } \\
\text { Others: } \\
\text { Com. Español } \\
\text { Jover }\end{array}$ & $\begin{array}{l}\quad \text { Bankinter } \\
\quad \text { Com. Español } \\
\text { Santander Negocios } \\
\text { Others: } \\
\text { Bank of America } \\
\text { Condal } \\
\text { Huelva (see Banesto } \\
\text { and Vizcaya) } \\
\text { Jover }\end{array}$ & \begin{tabular}{l}
\multicolumn{1}{c}{ Bankinter } \\
\multicolumn{1}{c}{ Santander Negocios } \\
$\quad$ Jover \\
Others: \\
Condal \\
Huelva
\end{tabular} & \\
\hline
\end{tabular}

(*) According to Anuario Estadístico de la Banca Privada, year 1990, Banco Santander group comprised Banco Santander de Negocios, Banco de Murcia and Banco Jover.

Table 12. Banco Popular

\begin{tabular}{|c|c|c|c|}
\hline 1947 & 1961 & 1966-67 & 1975 \\
\hline (see Central) & (see Central) & \begin{tabular}{l}
\multicolumn{1}{c}{ Andalucía (see } \\
$\quad$ Central) \\
$\quad$ Salamanca \\
$\quad$ Huelva \\
Europeo Negocios \\
Others: \\
La Coruña (see \\
Bilbao) \\
Ind. Cataluña (see \\
Banesto and Central) \\
Zaragozano \\
López Quesada \\
Central
\end{tabular} & $\begin{array}{l}\text { Europeo Negocios } \\
\\
\text { Others: } \\
\text { Créd. Balear } \\
\text { Andalucía } \\
\text { Castilla } \\
\text { Societe Generale }\end{array}$ \\
\hline 1980 & 1985 & $1990(*)$ & \\
\hline $\begin{array}{l}\quad \text { Popular Ind. (ex- } \\
\text { Europeo Negocios) } \\
\text { Others: } \\
\text { Créd. Balear } \\
\text { Andalucía }\end{array}$ & $\begin{array}{l}\quad \text { Popular Ind. } \\
\text { Others: } \\
\text { Créd. Balear } \\
\text { Andalucía } \\
\text { Exportación }\end{array}$ & $\begin{array}{l}\quad \text { Popular Ind. } \\
\text { Others: } \\
\text { Créd. Balear } \\
\text { Andalucía }\end{array}$ & \\
\hline
\end{tabular}

(*) According to Anuario Estadístico de la Banca Privada, year 1990, Banco Popular group comprised Banco de Andalucía, Banco de Castilla, Banco de Crédito Balear, Banco de Galicia, Banco Vasconia and Banco Popular Industrial. 


\section{Banco Hispano Americano}
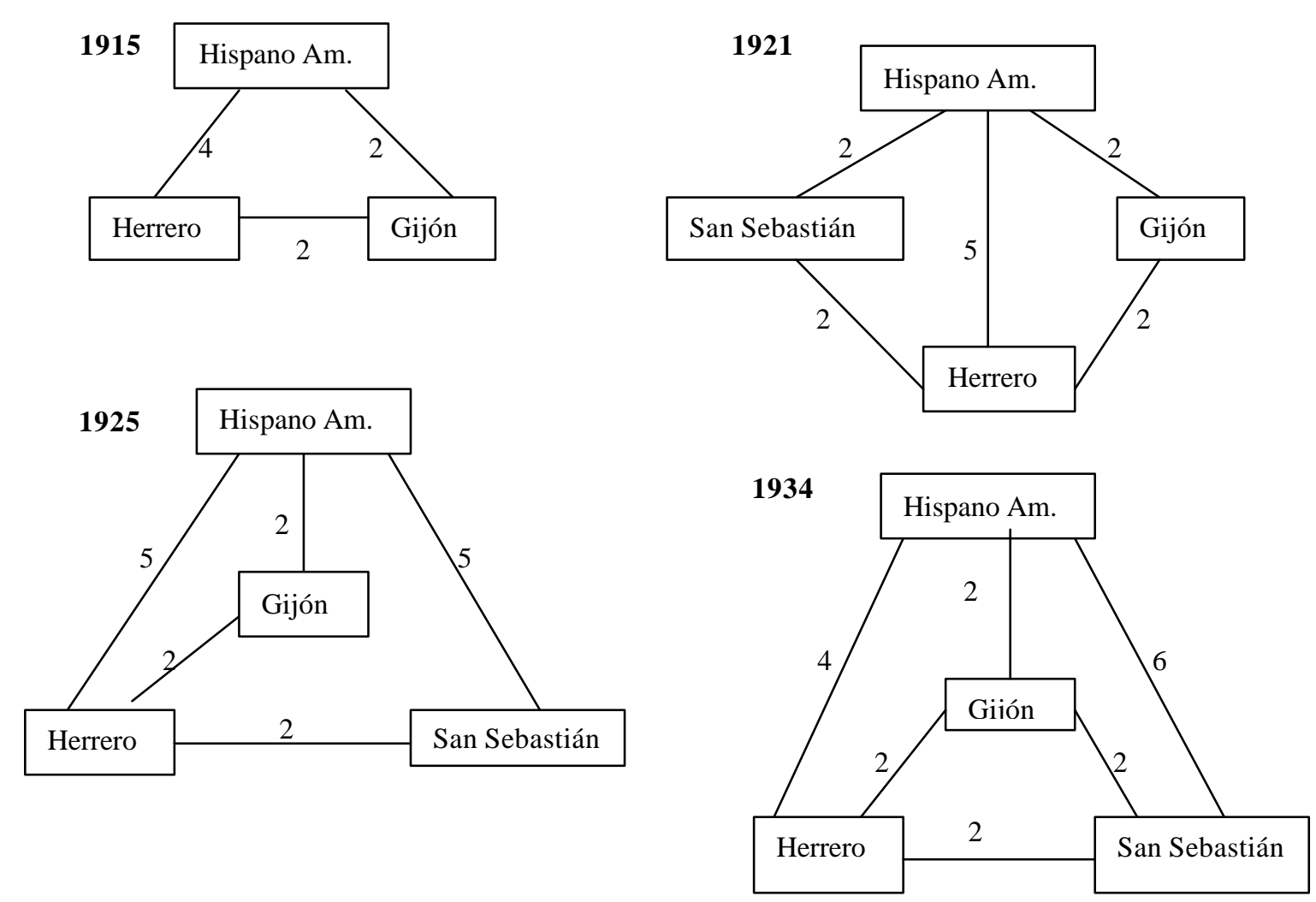

1947

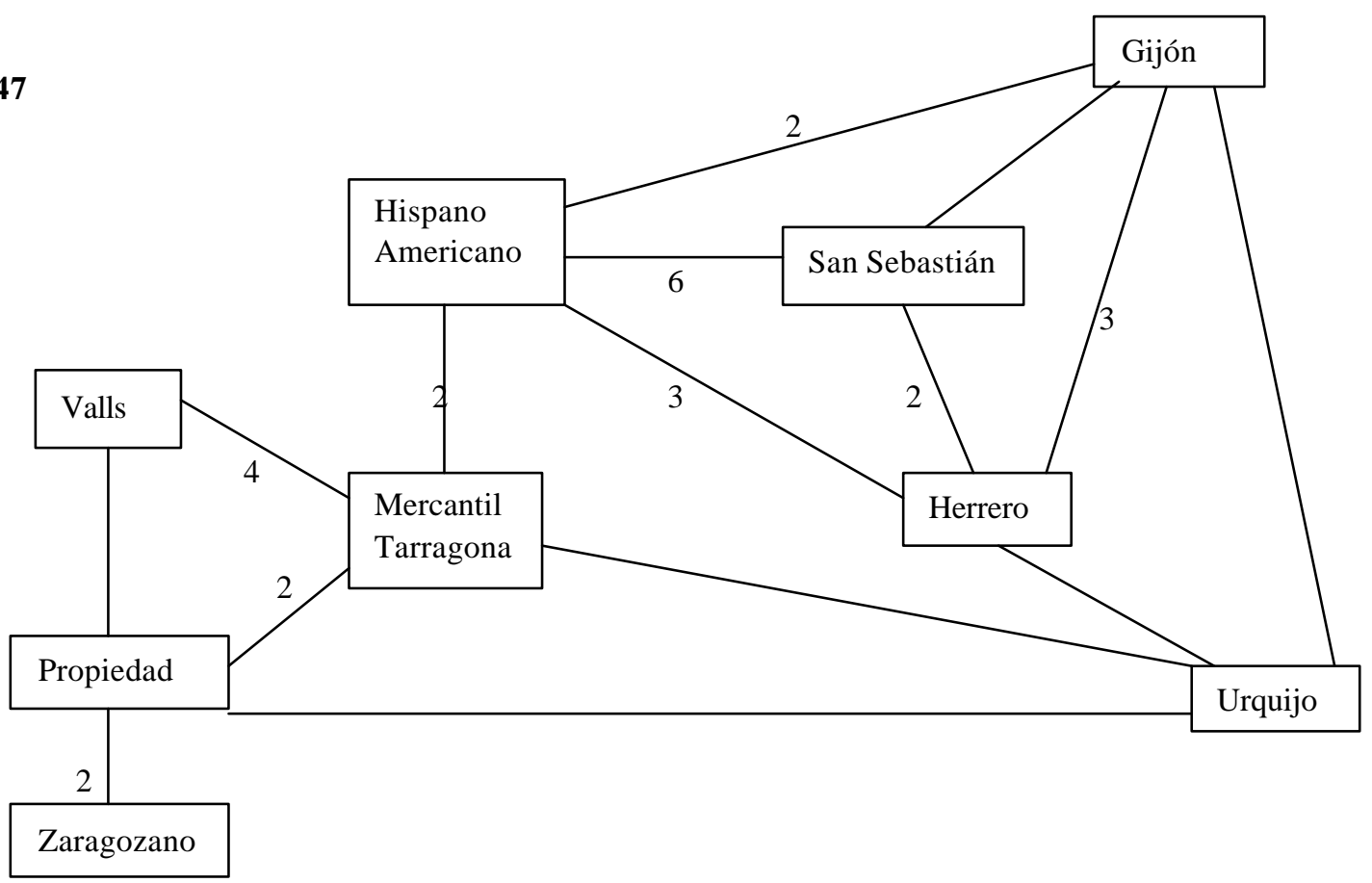




\section{Banco Hispano Americano}
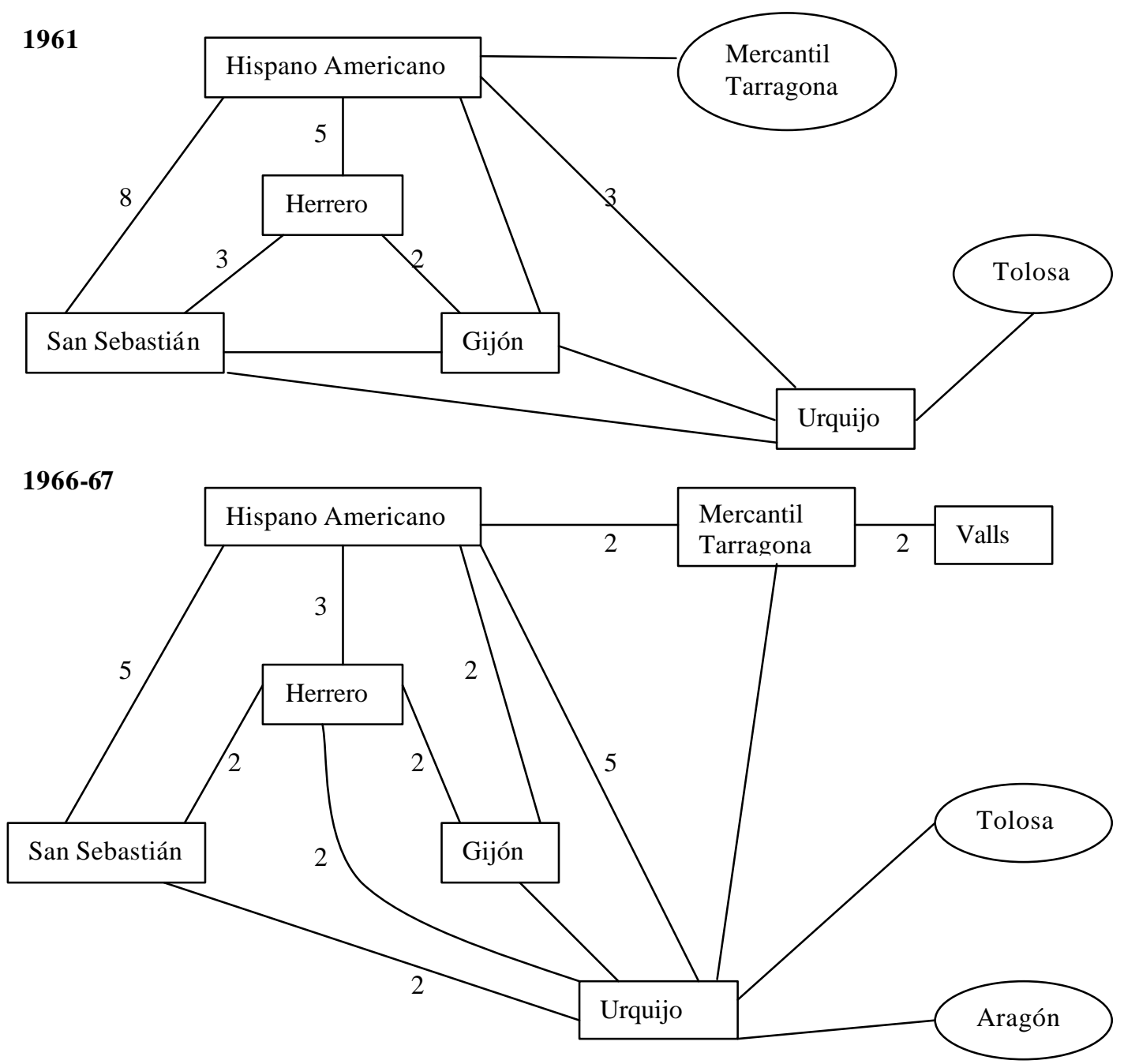

1975

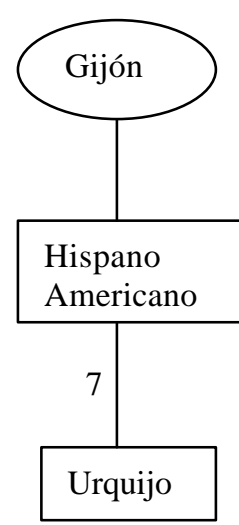

1980

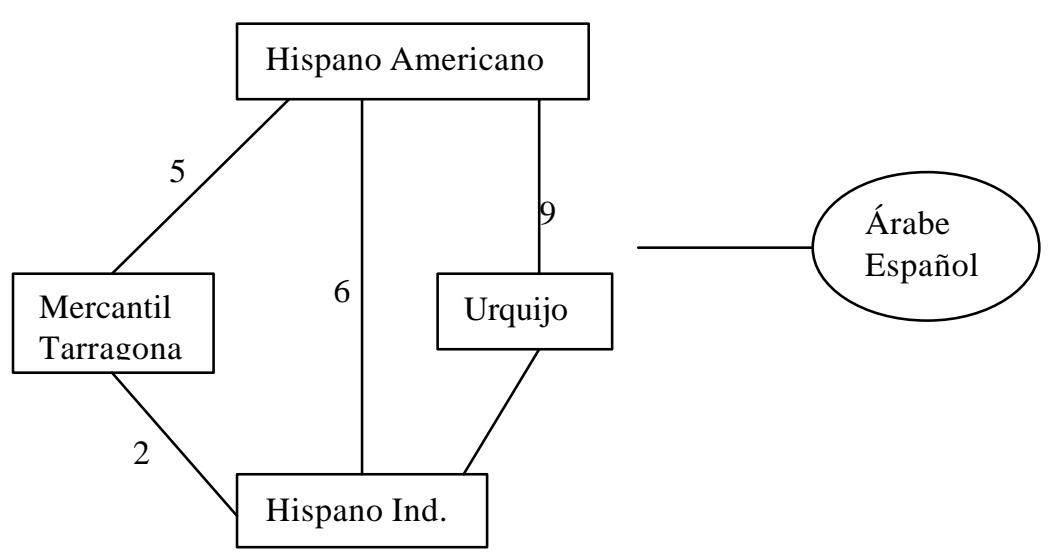




\section{Banco Hispano Americano}

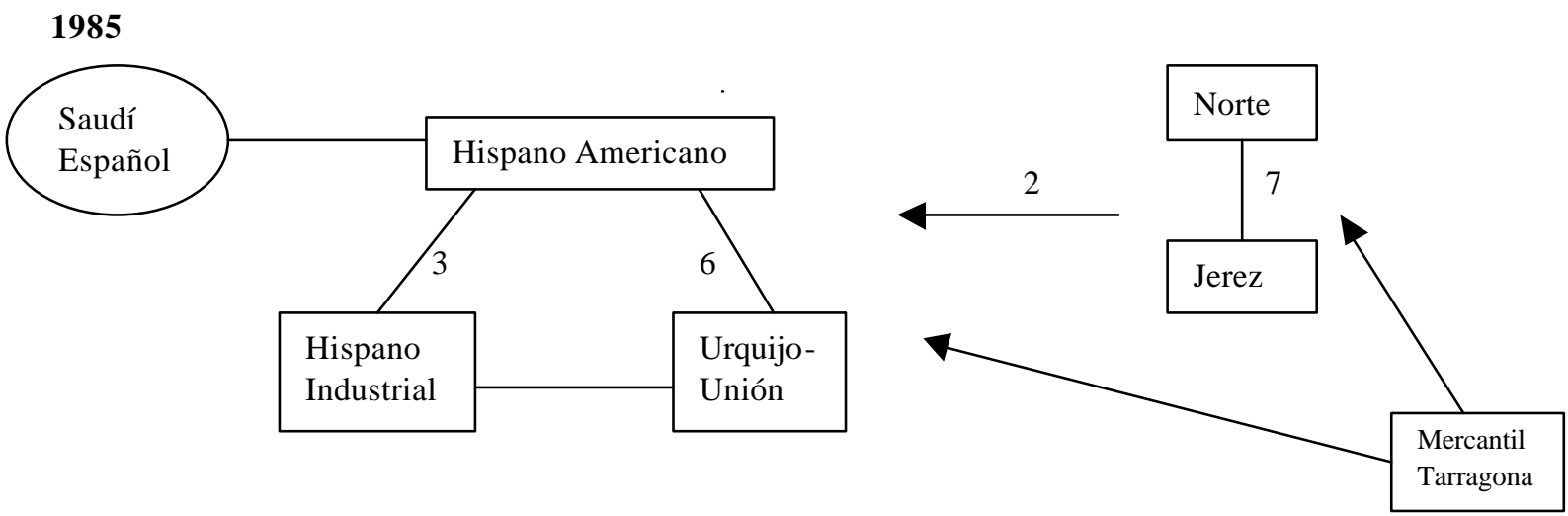

1990

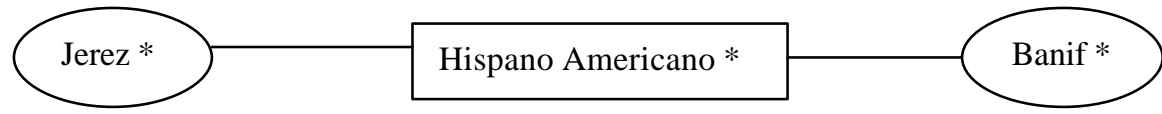

Mercantil

Tarragona *

\section{Banco Urquijo}

1921

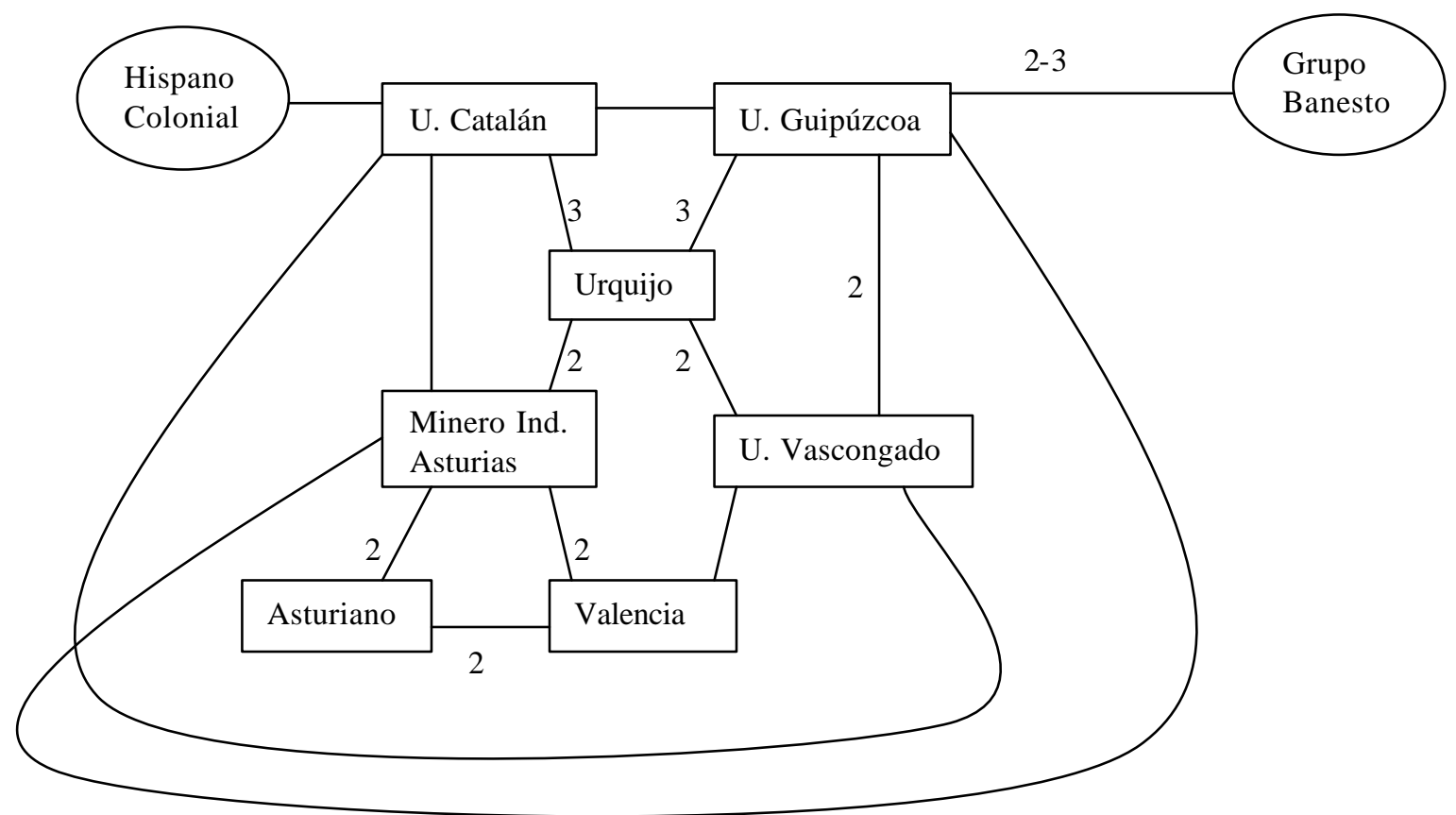




\section{Banco Urquijo}

1925

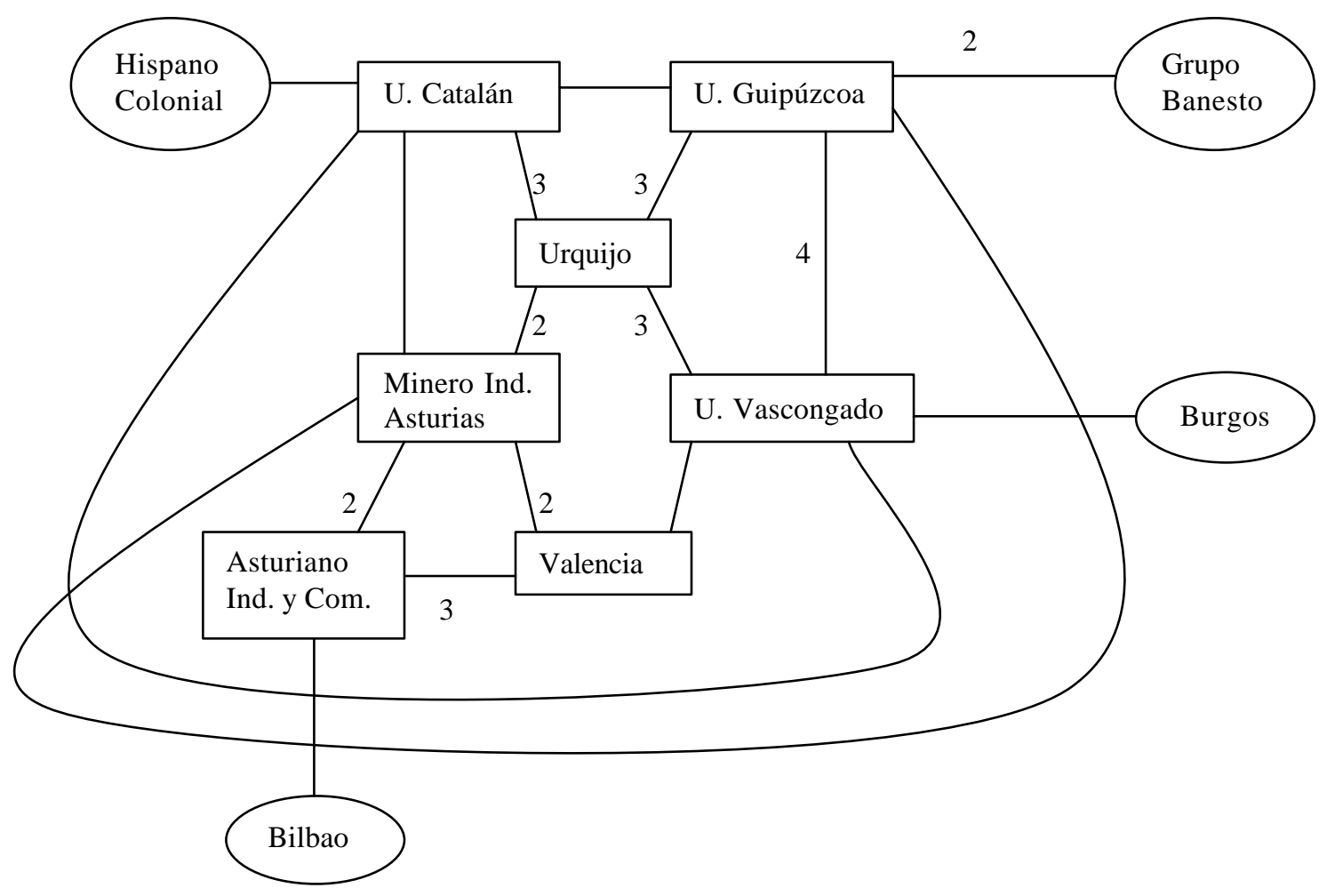

1934

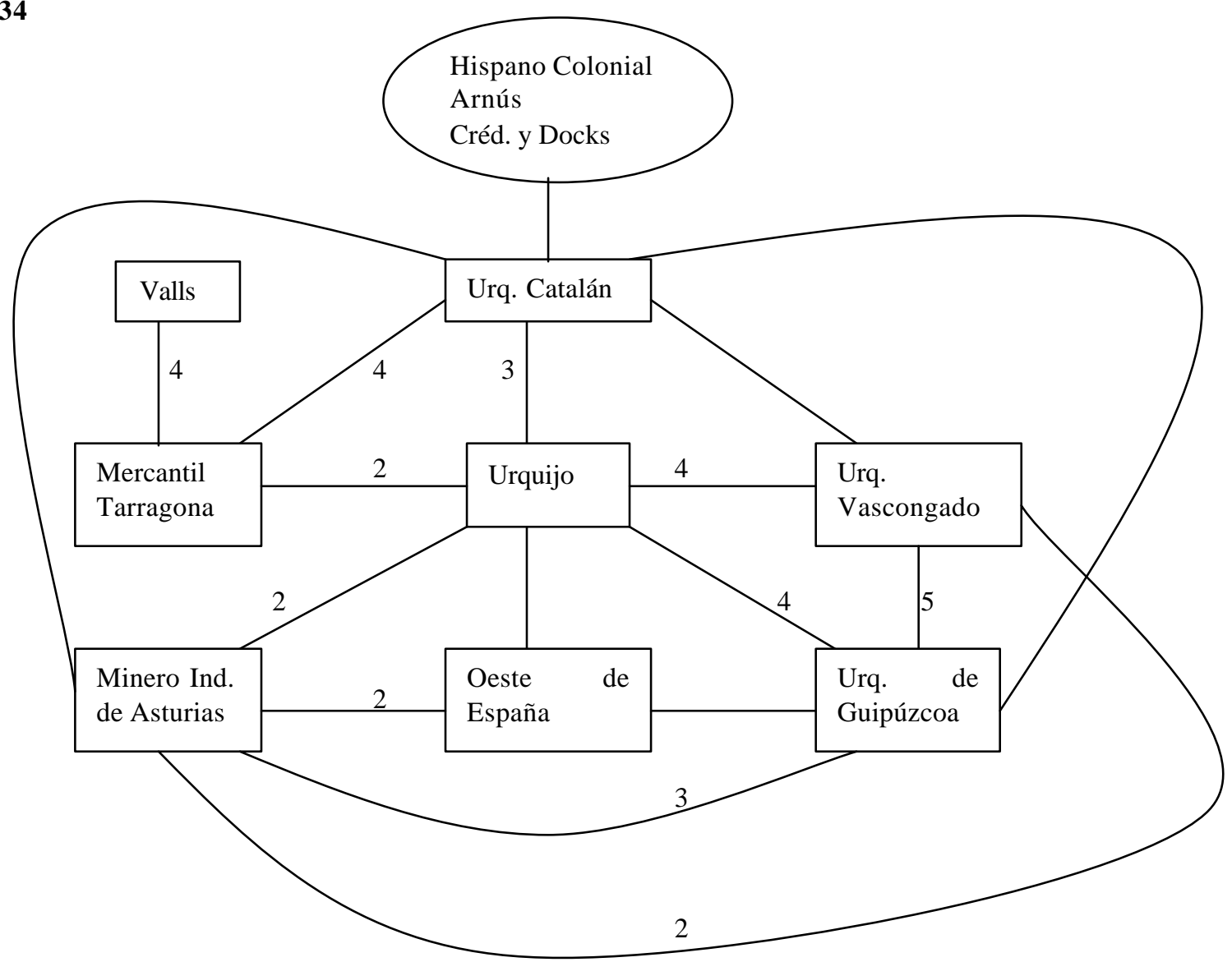




\section{Banco Español de Crédito}

1915

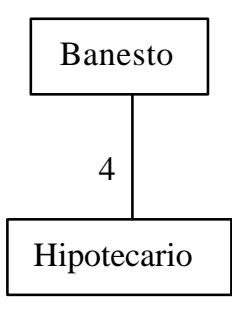

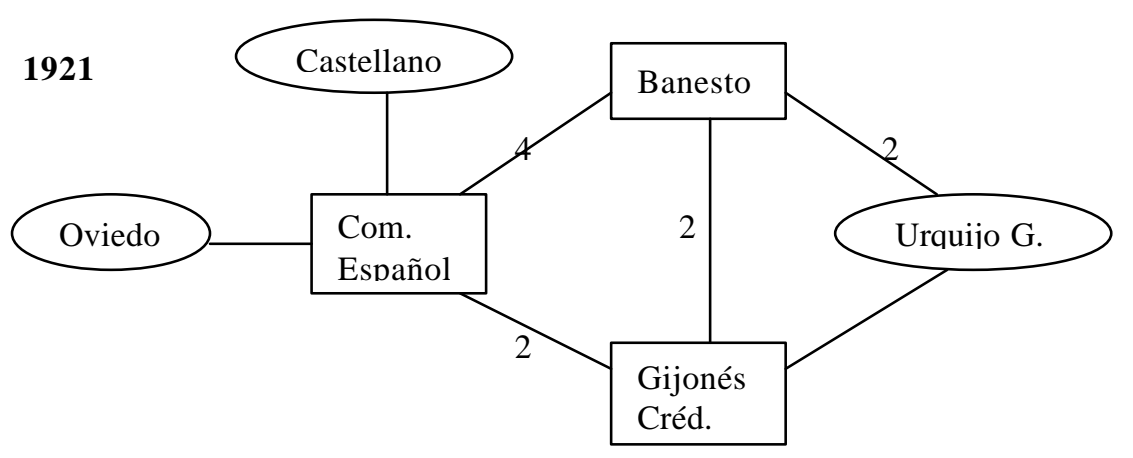
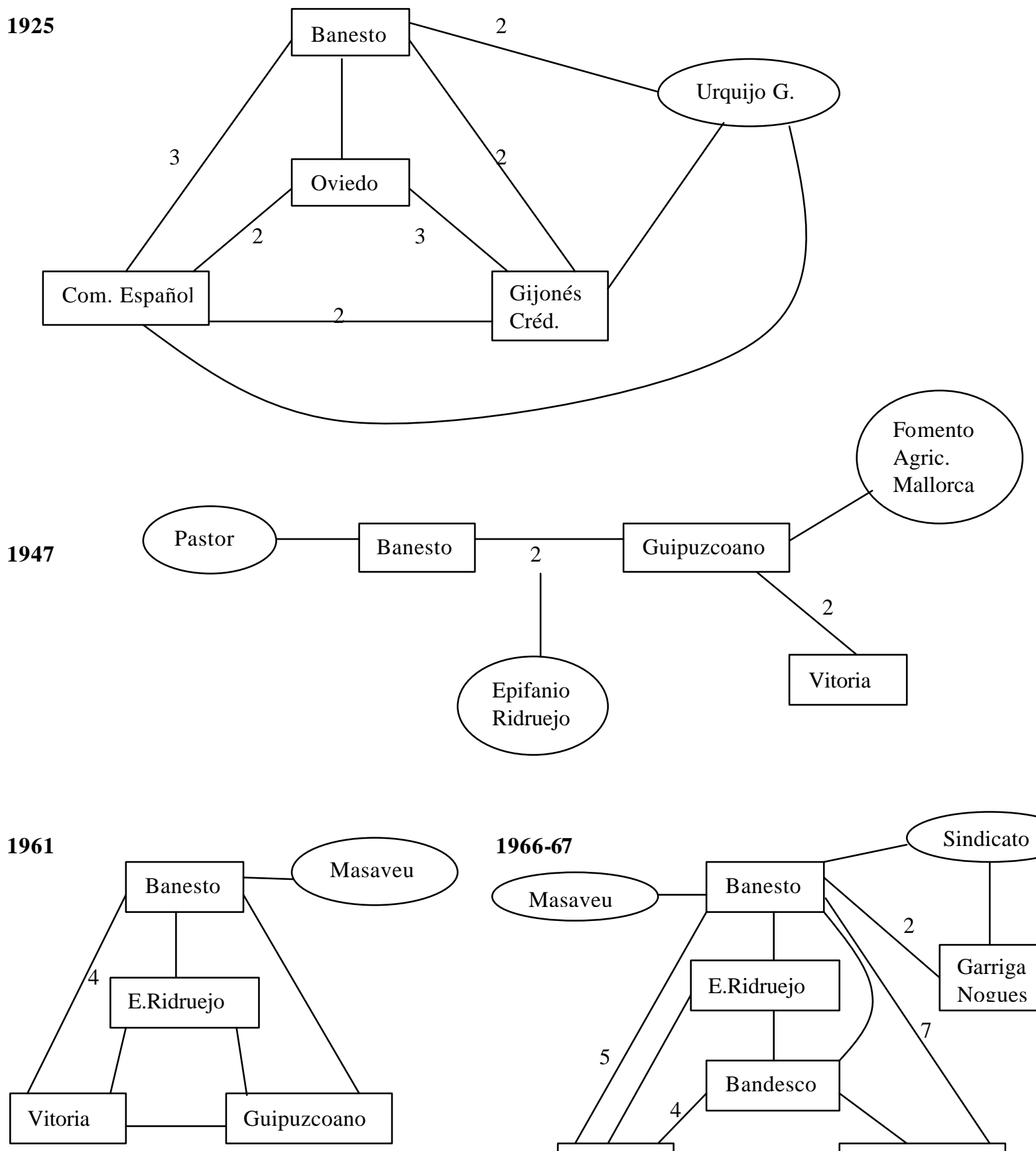

1966-67

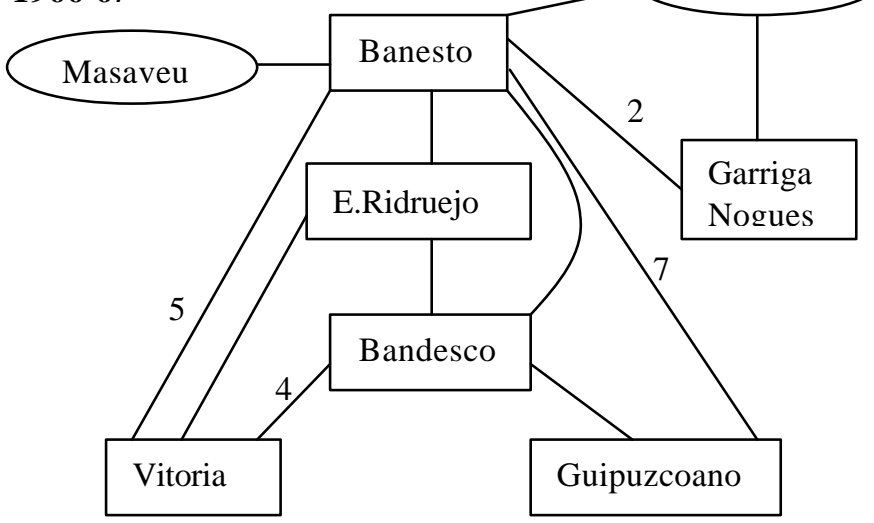




\section{Banco Español de Crédito}
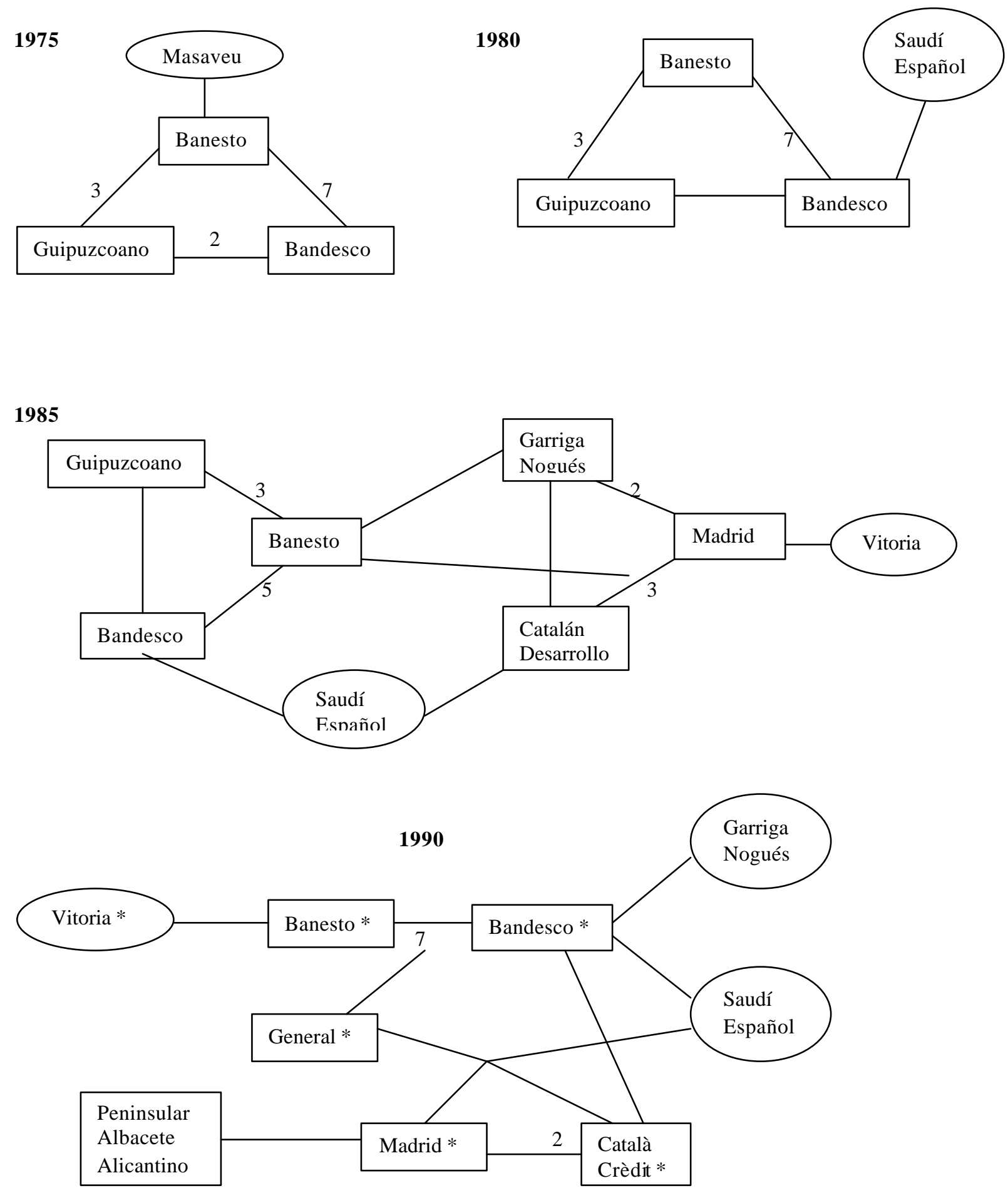


\section{Banco Central}

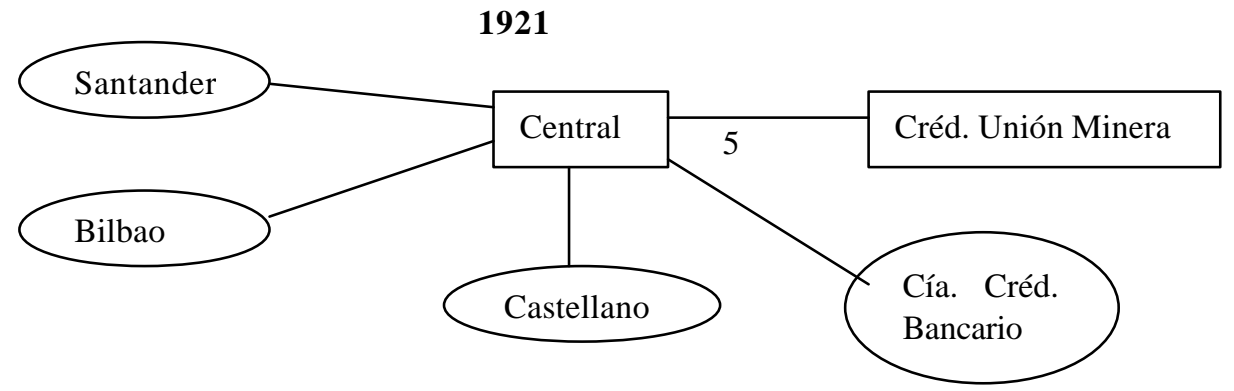

1925

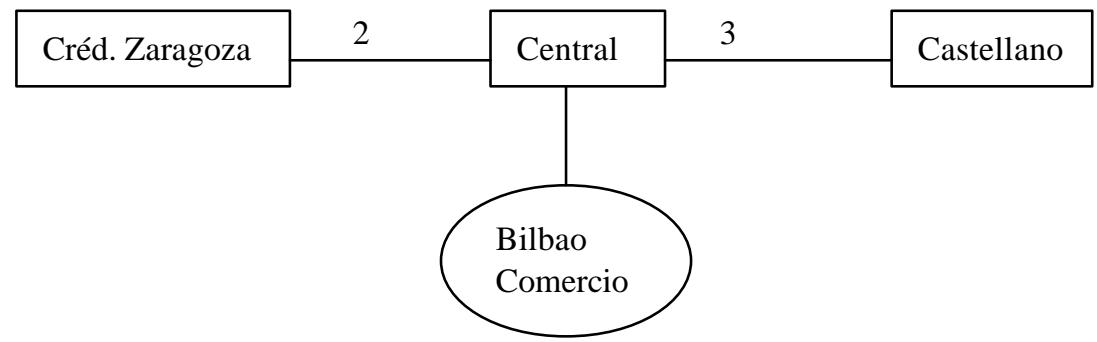

1934

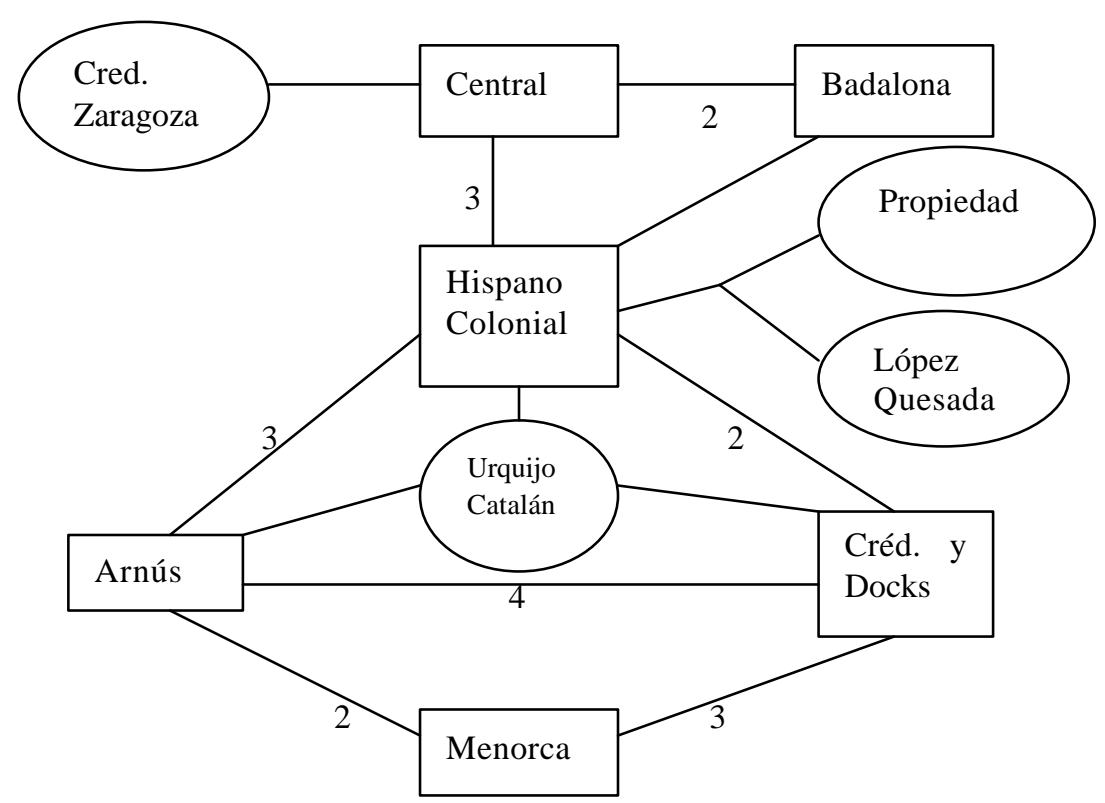




\section{Banco Central}

1947

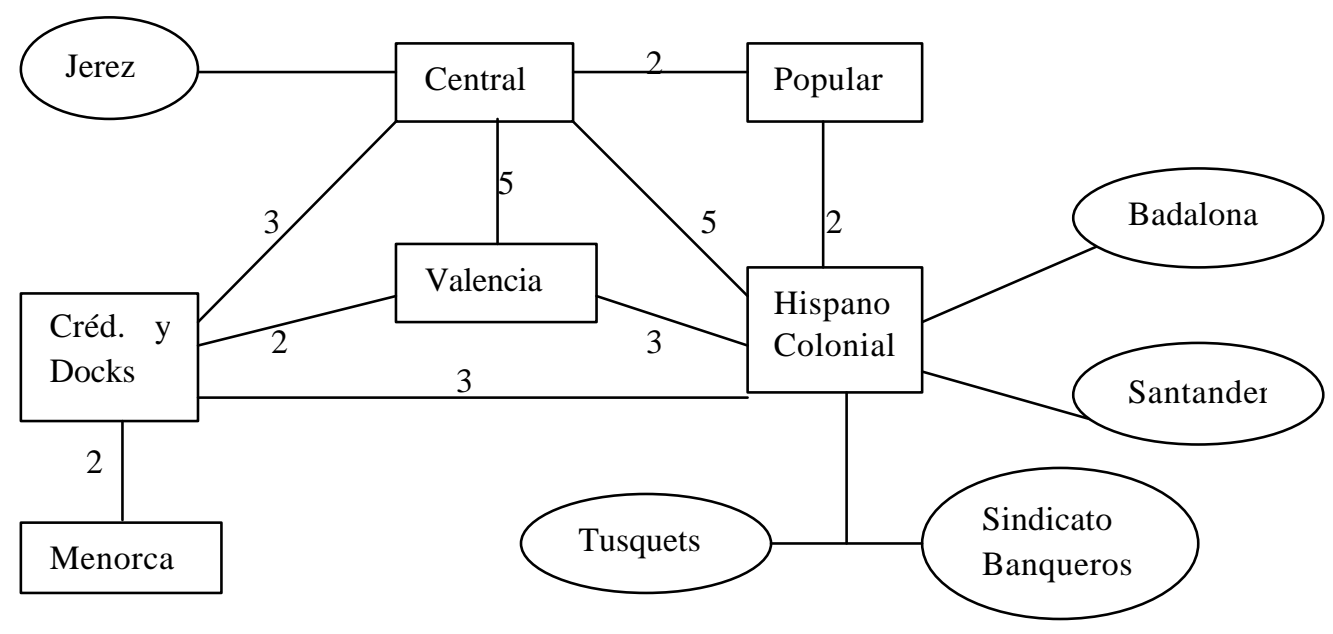

1961
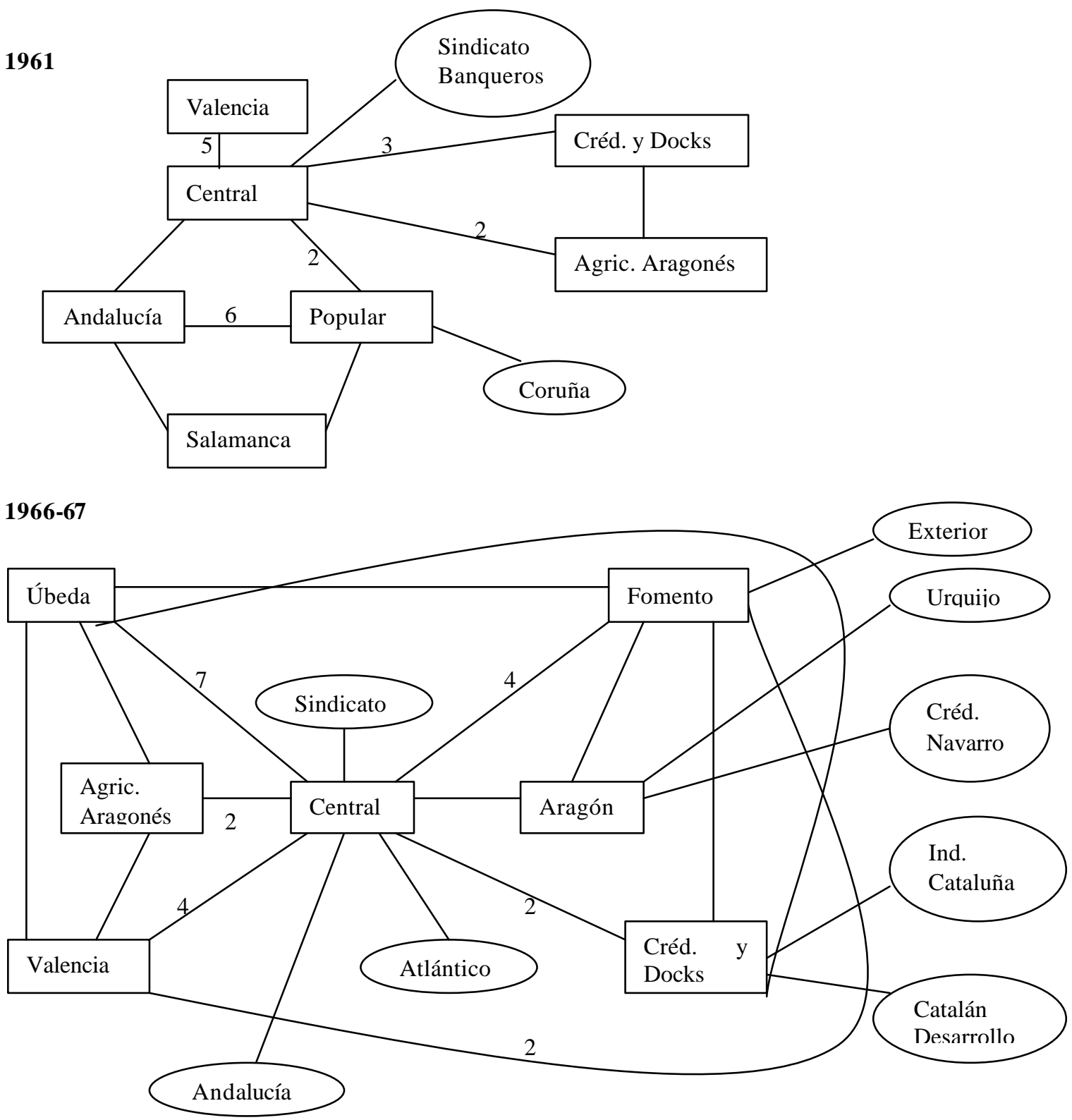


\section{Banco Central}

1975

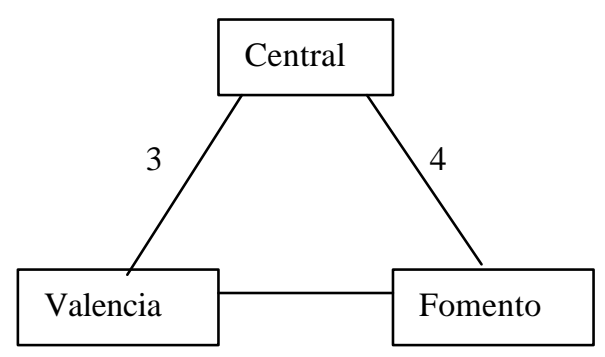

1980

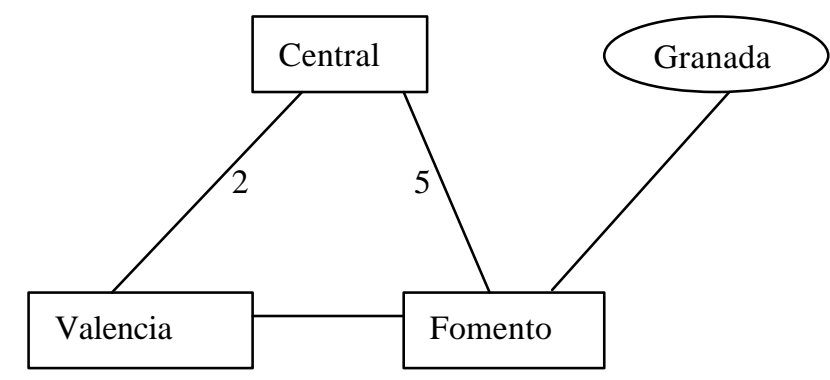

1985

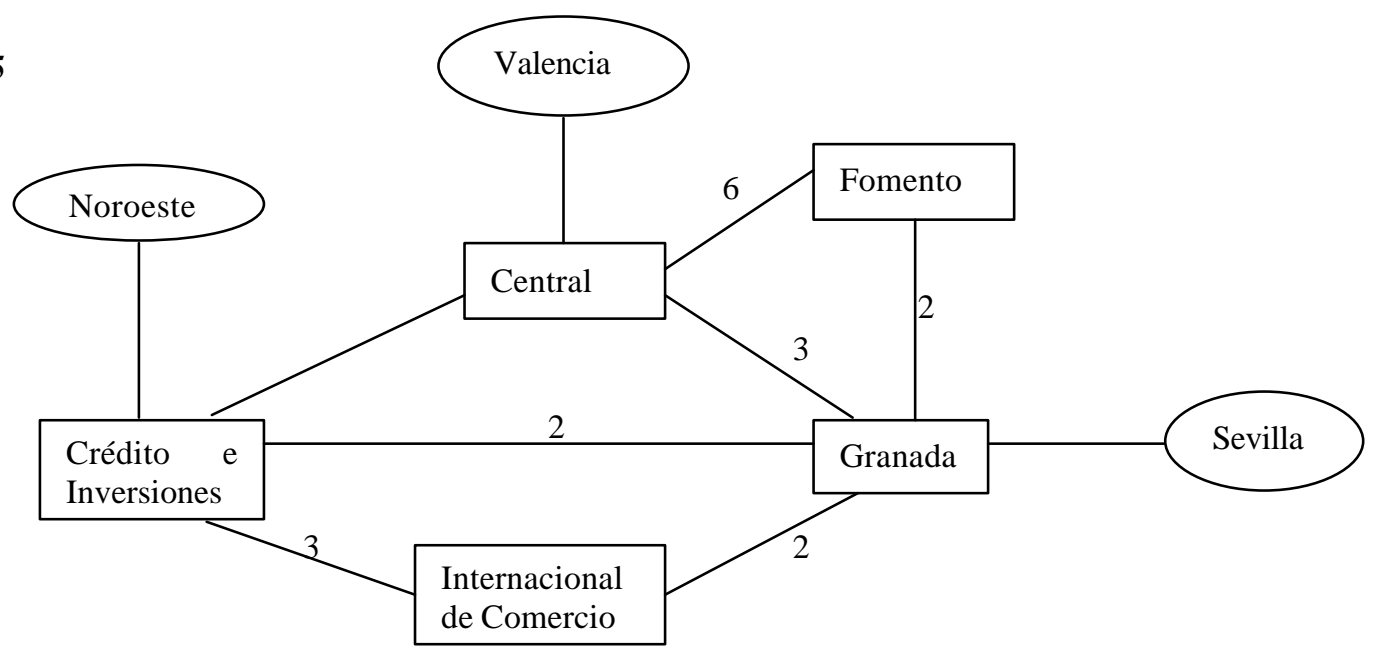

1990

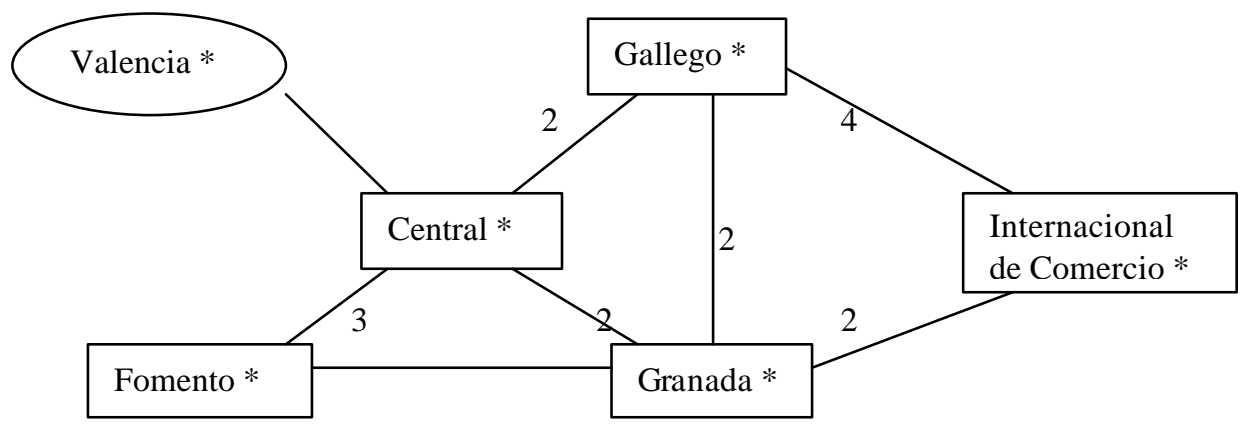




\section{Banco de Bilbao}

1915

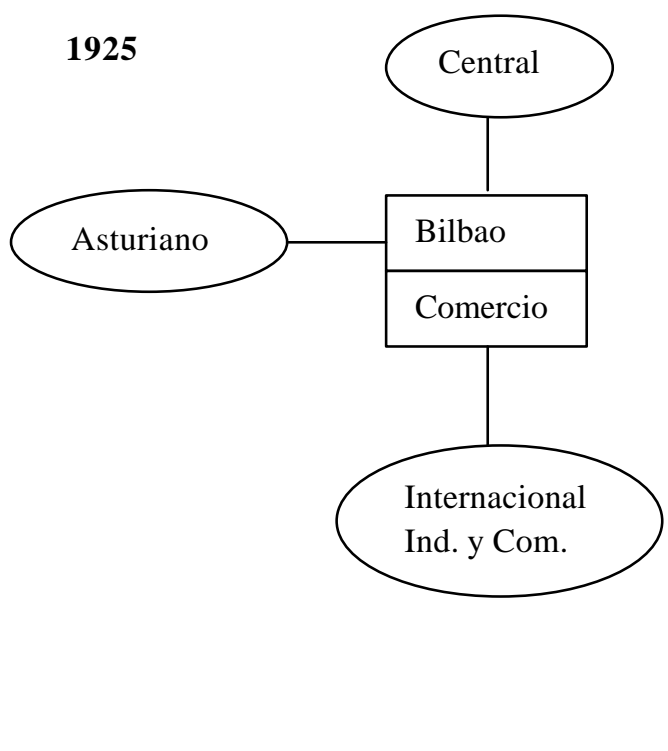

1947

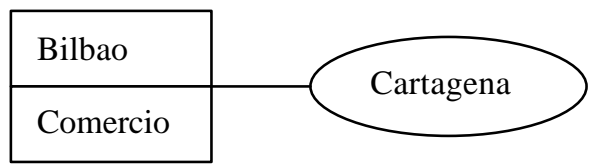

1934

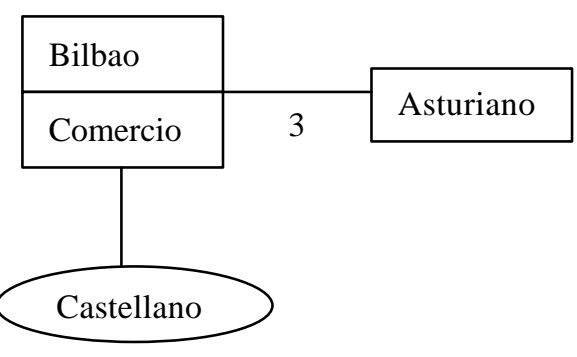

Ind. y Com.

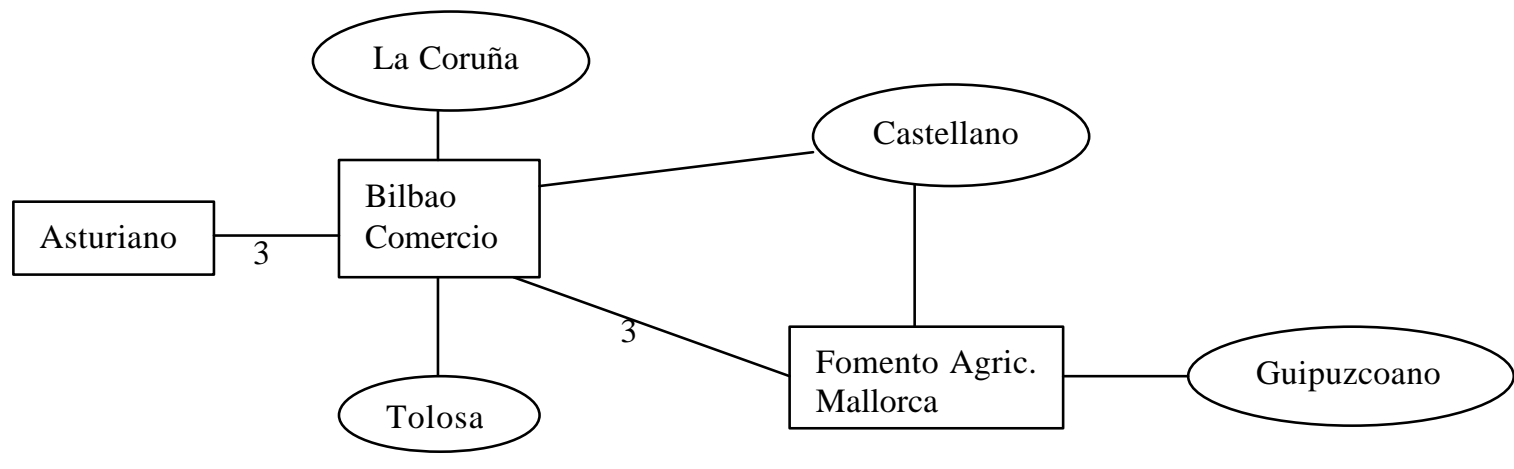

1961

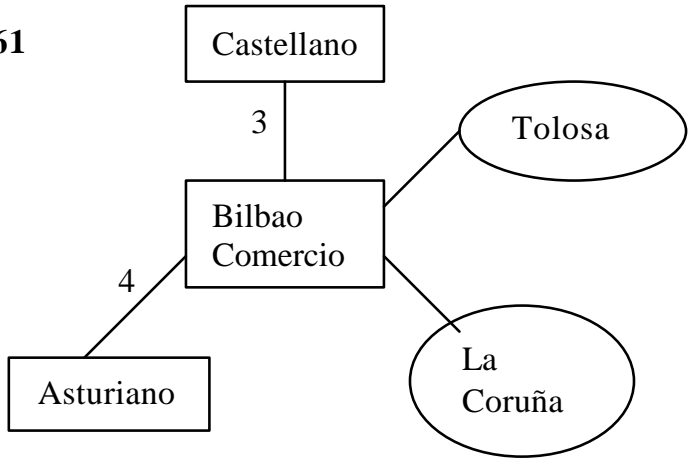

1975

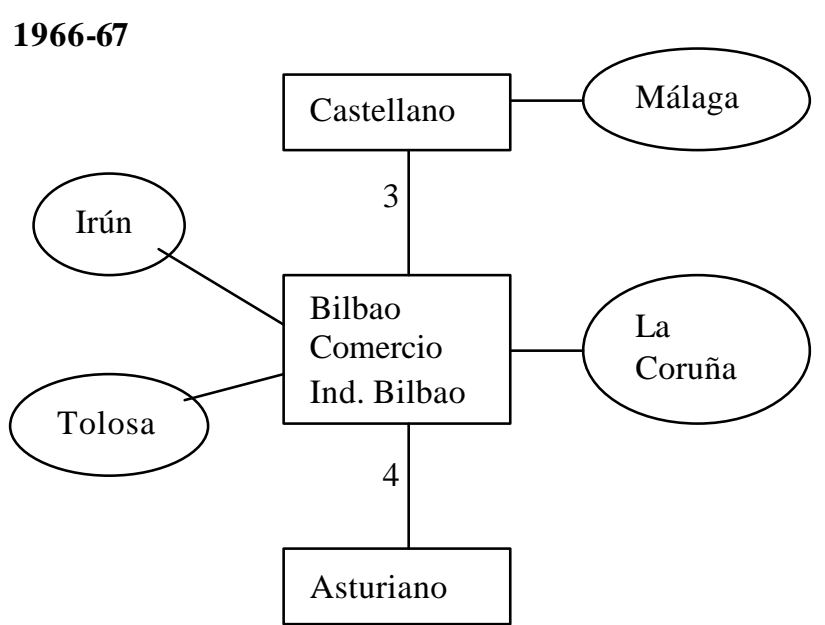




\section{Banco de Bilbao}

1980

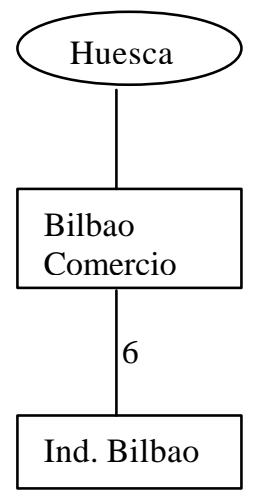

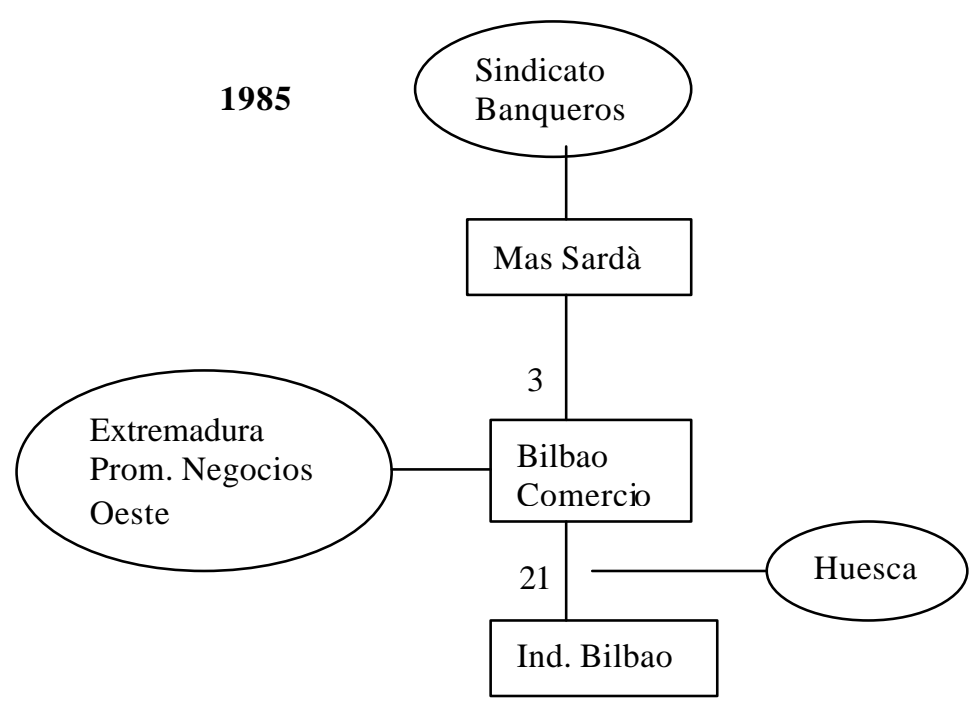

\section{Banco de Bilbao-Vizcaya}

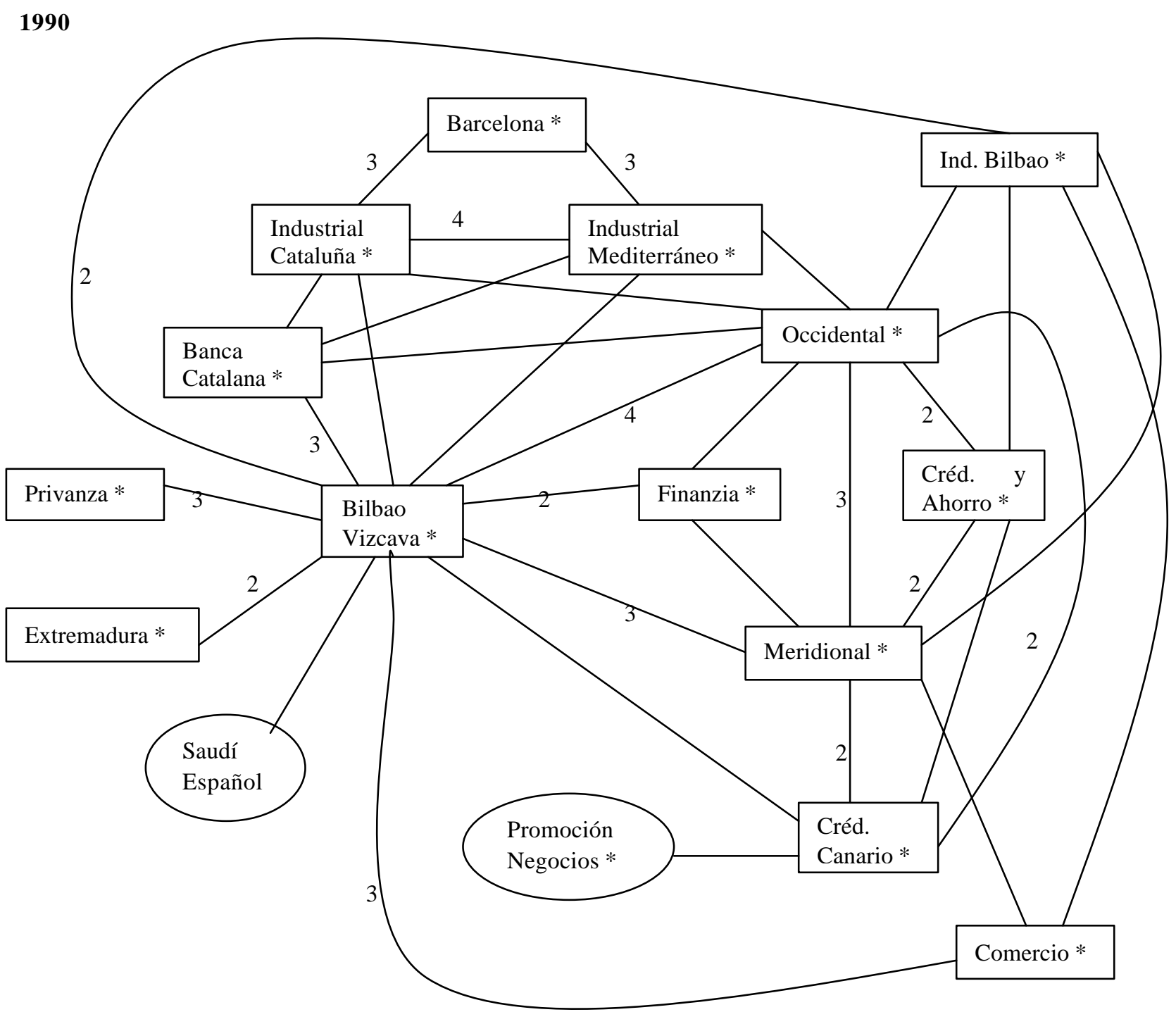




\section{Banco de Vizcaya}

1975

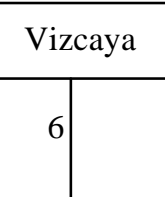

Financiación Ind.
1980

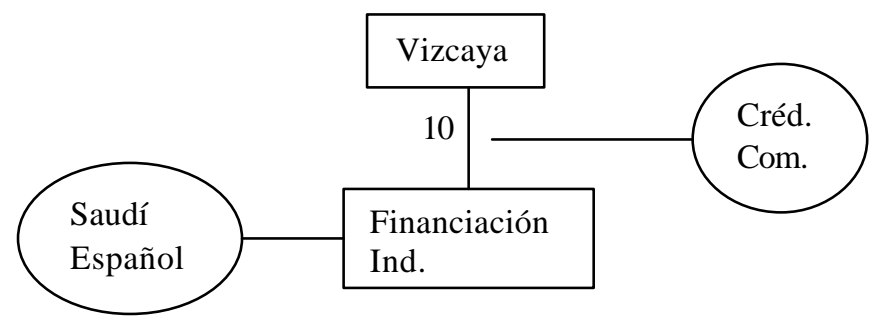

1985

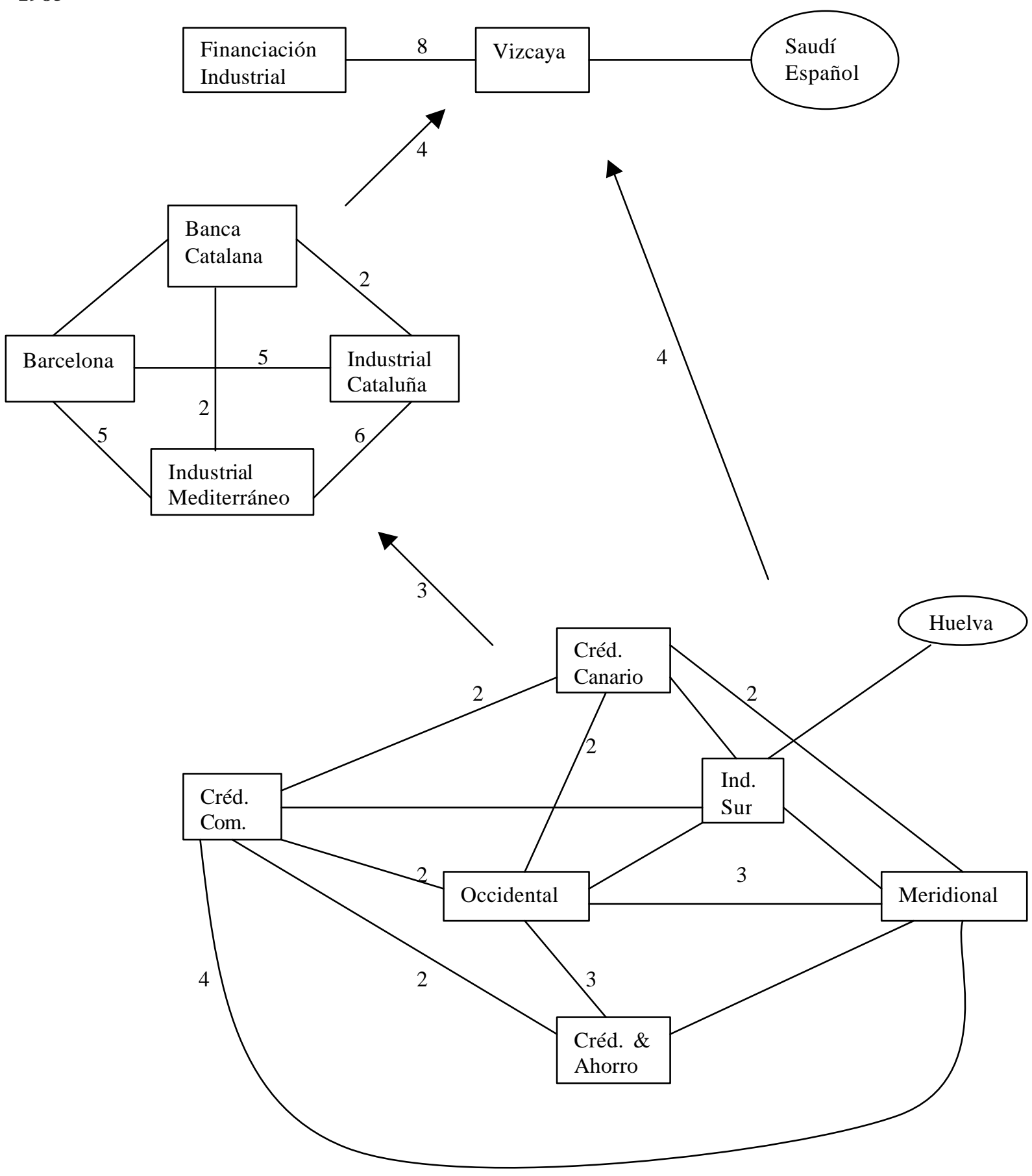




\section{Catalan banks before civil war}

1915
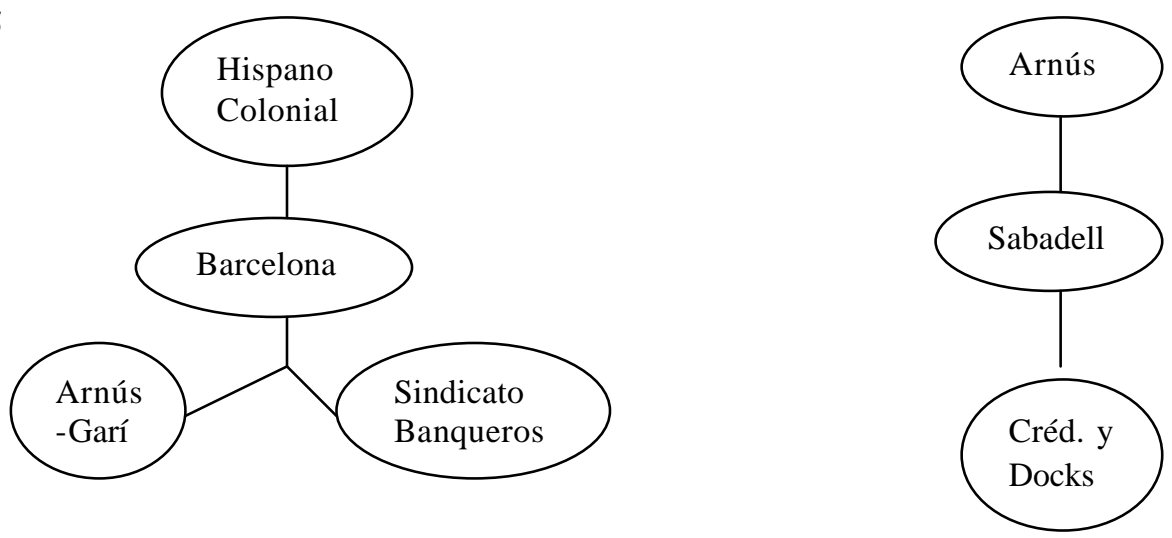

1921

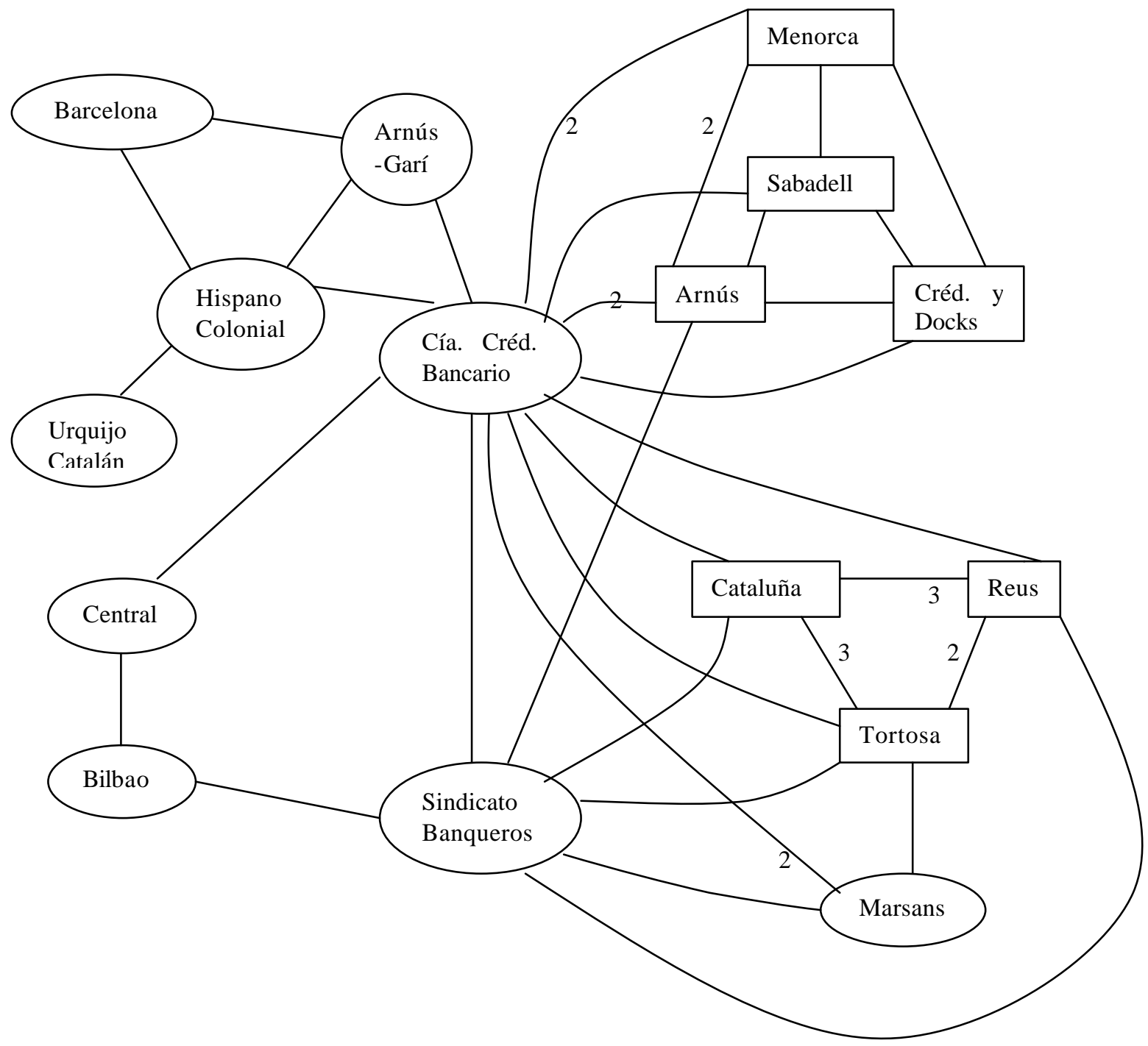




\section{Catalan banks before civil war}

1925

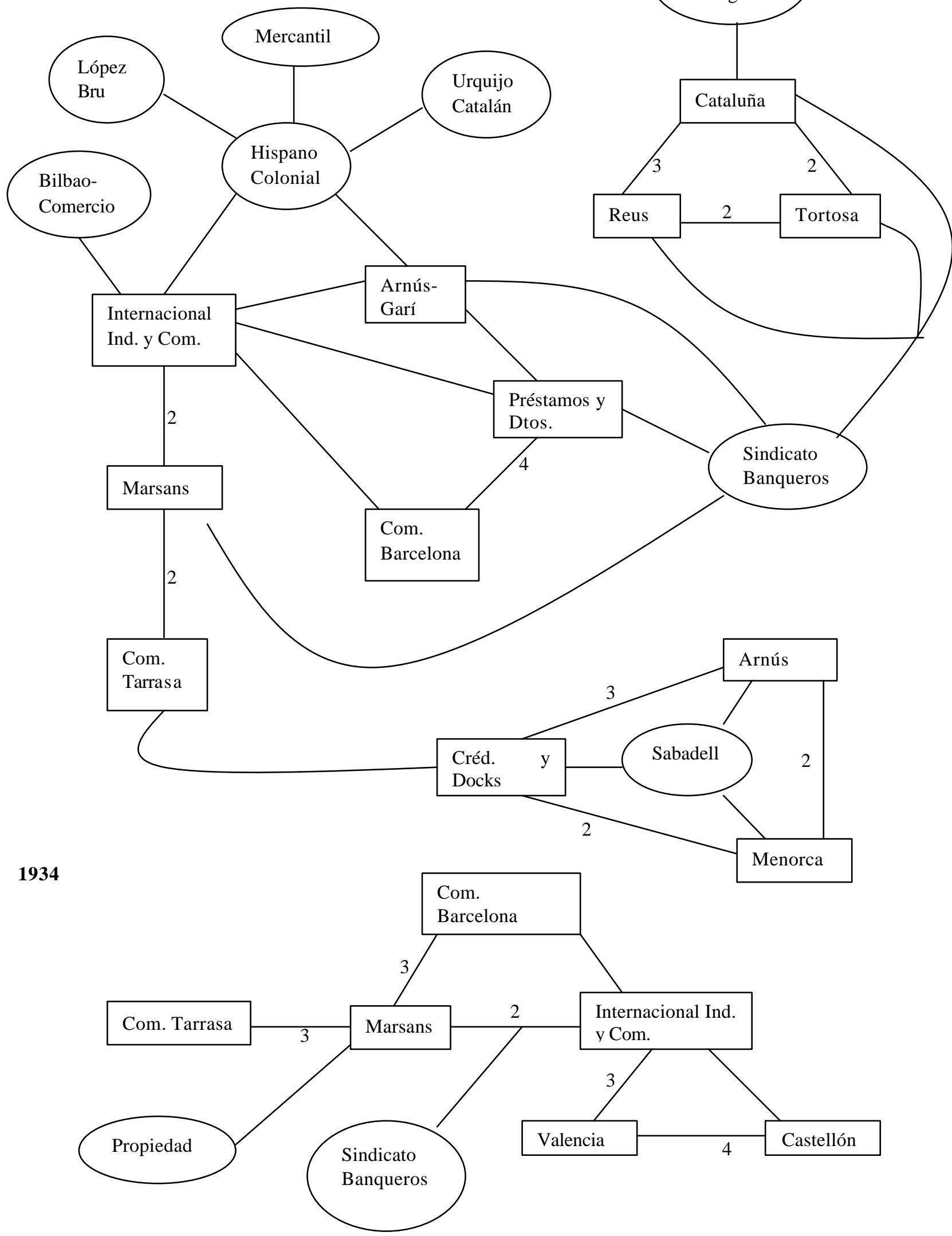




\section{Banco de Santander}

1961

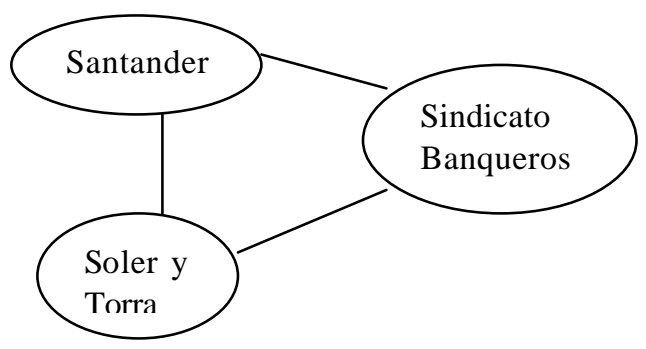

1975

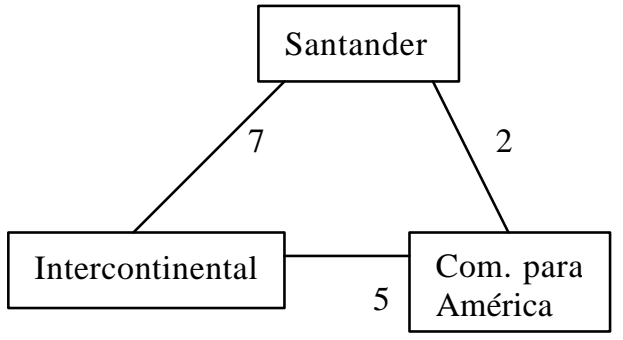

1966-67

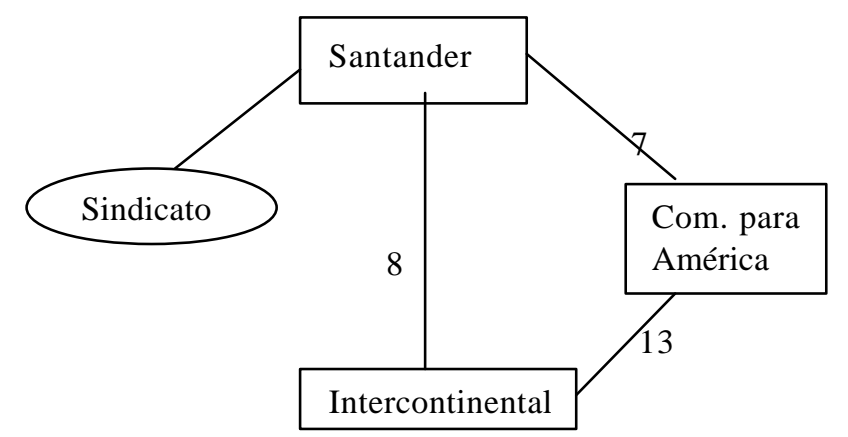

1980

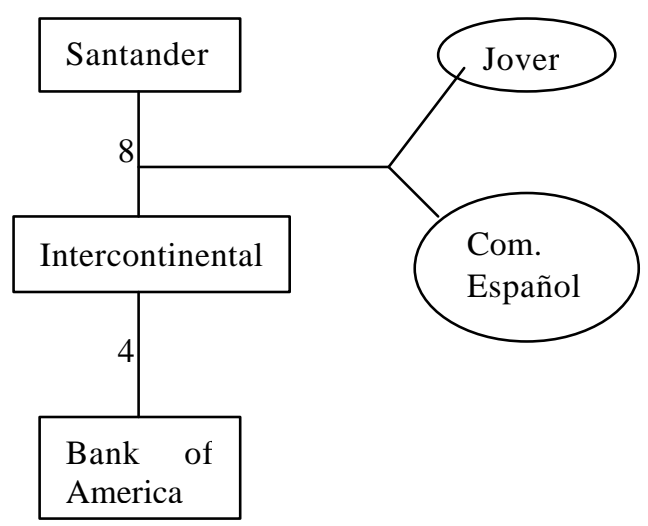

1990

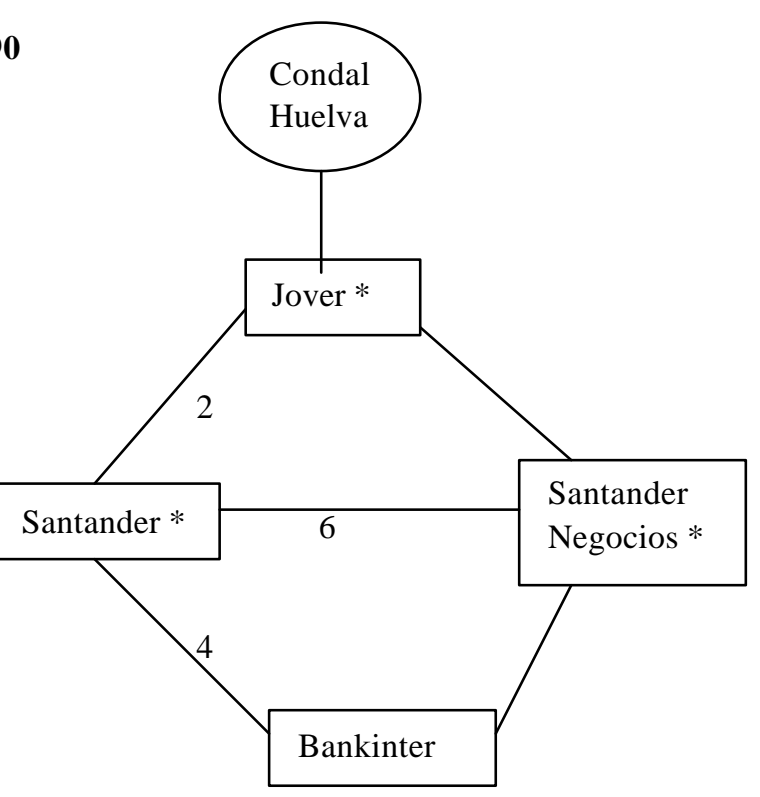

Bankinter
1985

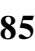

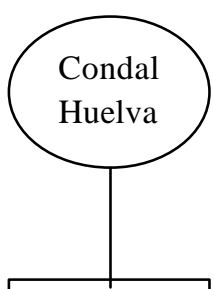

Santander Negocios

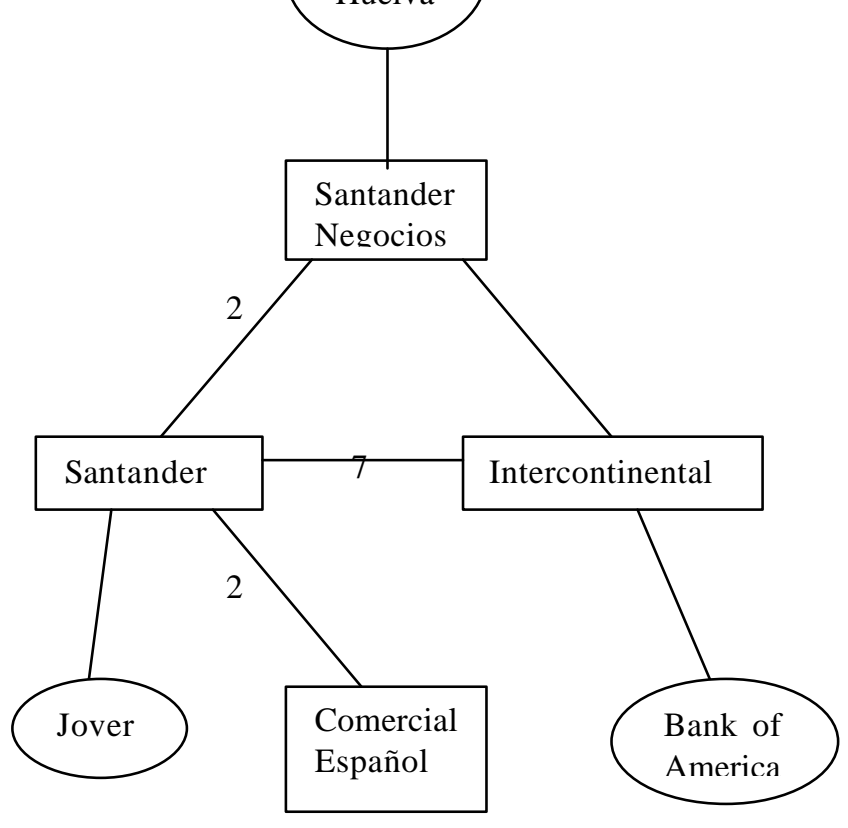

Intercontinental

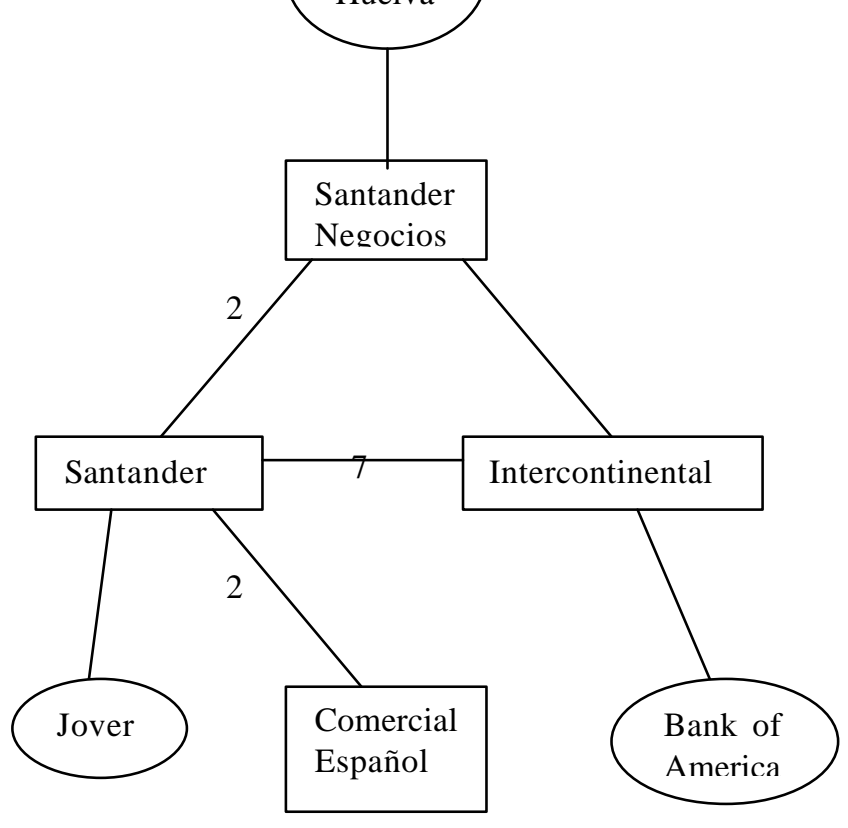




\section{Banco Popular}
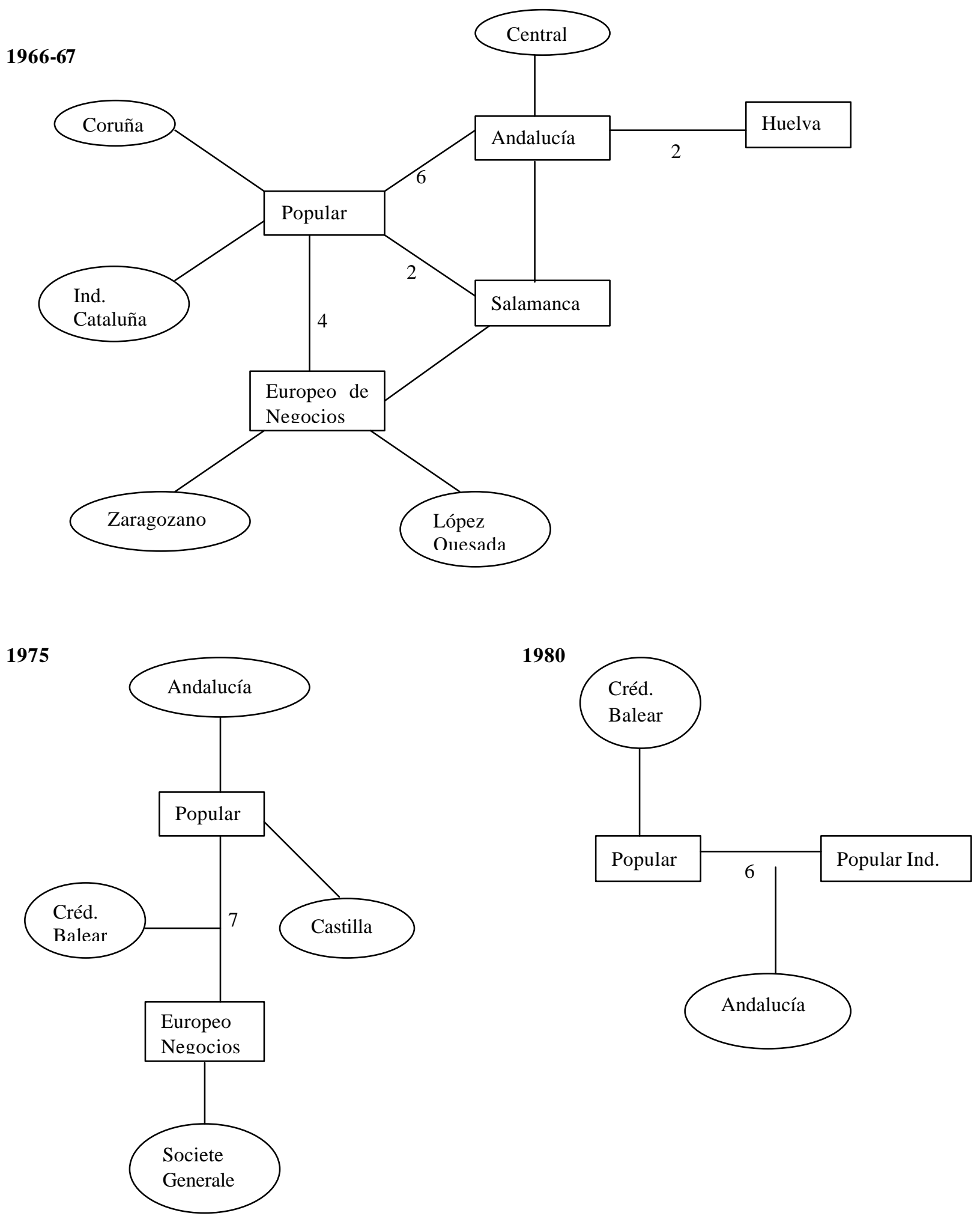


\section{Banco Popular}

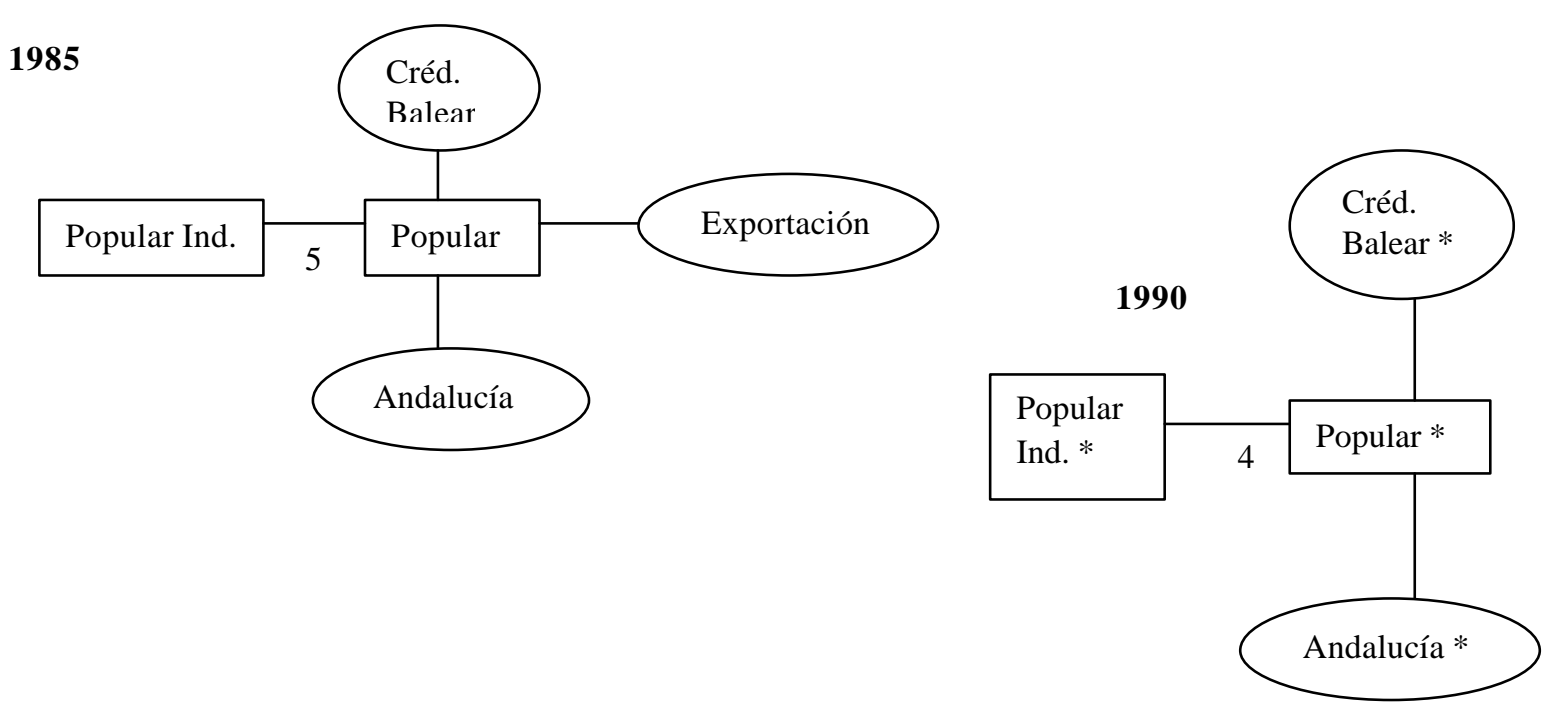




\section{Appendix 3. Diagrams of connections between groups}

\section{Connections in 1921}

Without links: Hispano Americano and Vizcaya

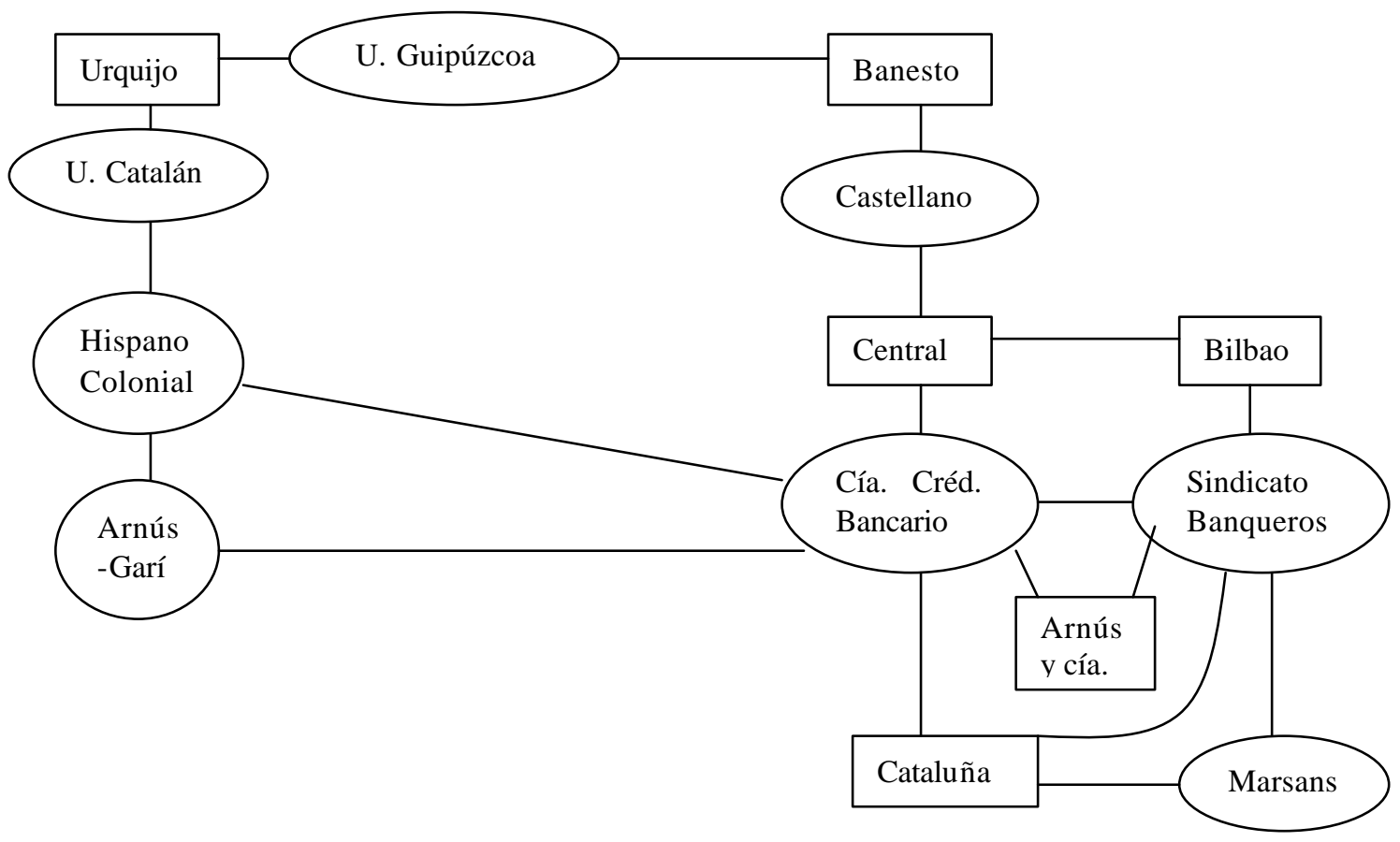

Connections in $\mathbf{1 9 2 5}$

Without links: Hispano Americano and Vizcaya

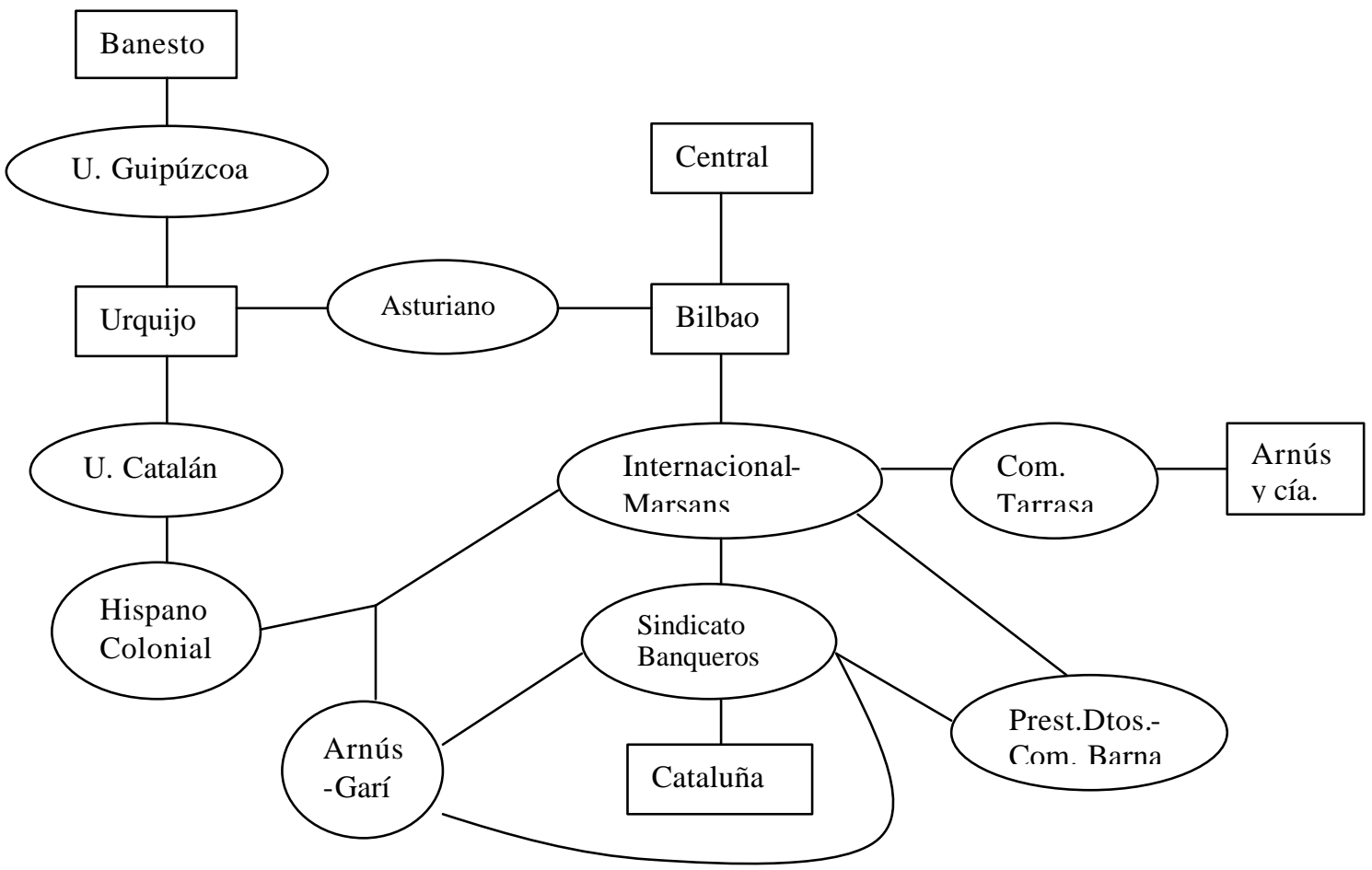


Connections in 1934

Without links: Hispano Americano, Banesto, Bilbao, and Vizcaya

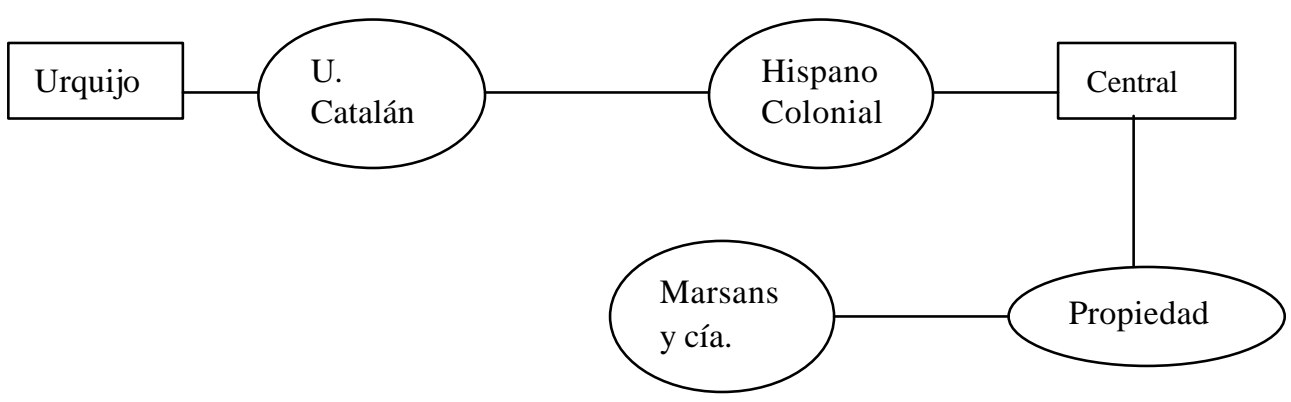

Connections in 1946

Without links: Hispano Americano, Central, and Vizcaya

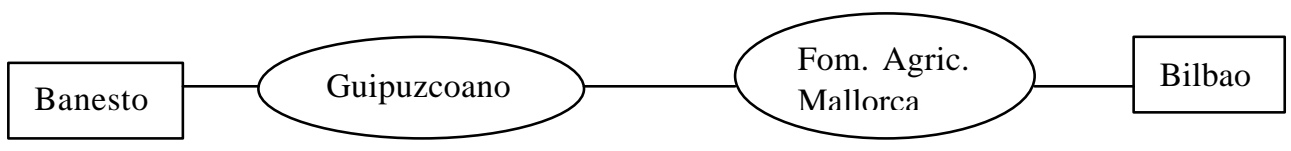

\section{Connections in 1961}

Without links: Banesto and Vizcaya

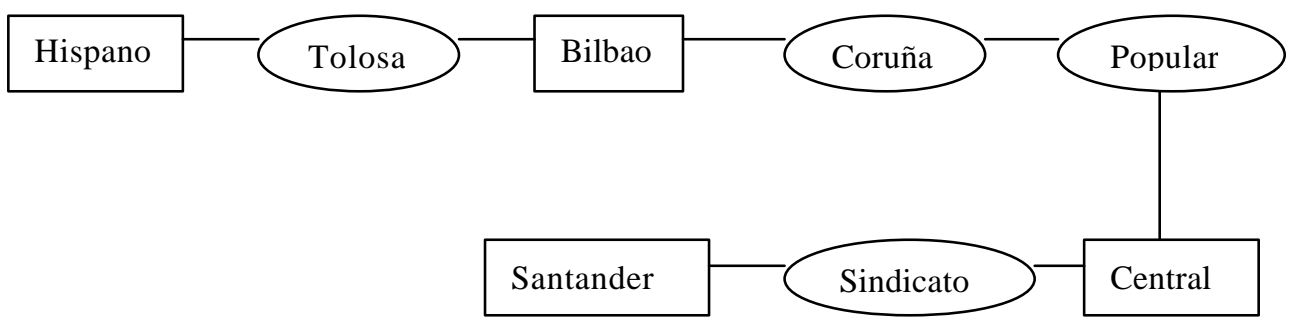




\section{Connections in 1966-67}

Without links: Vizcaya

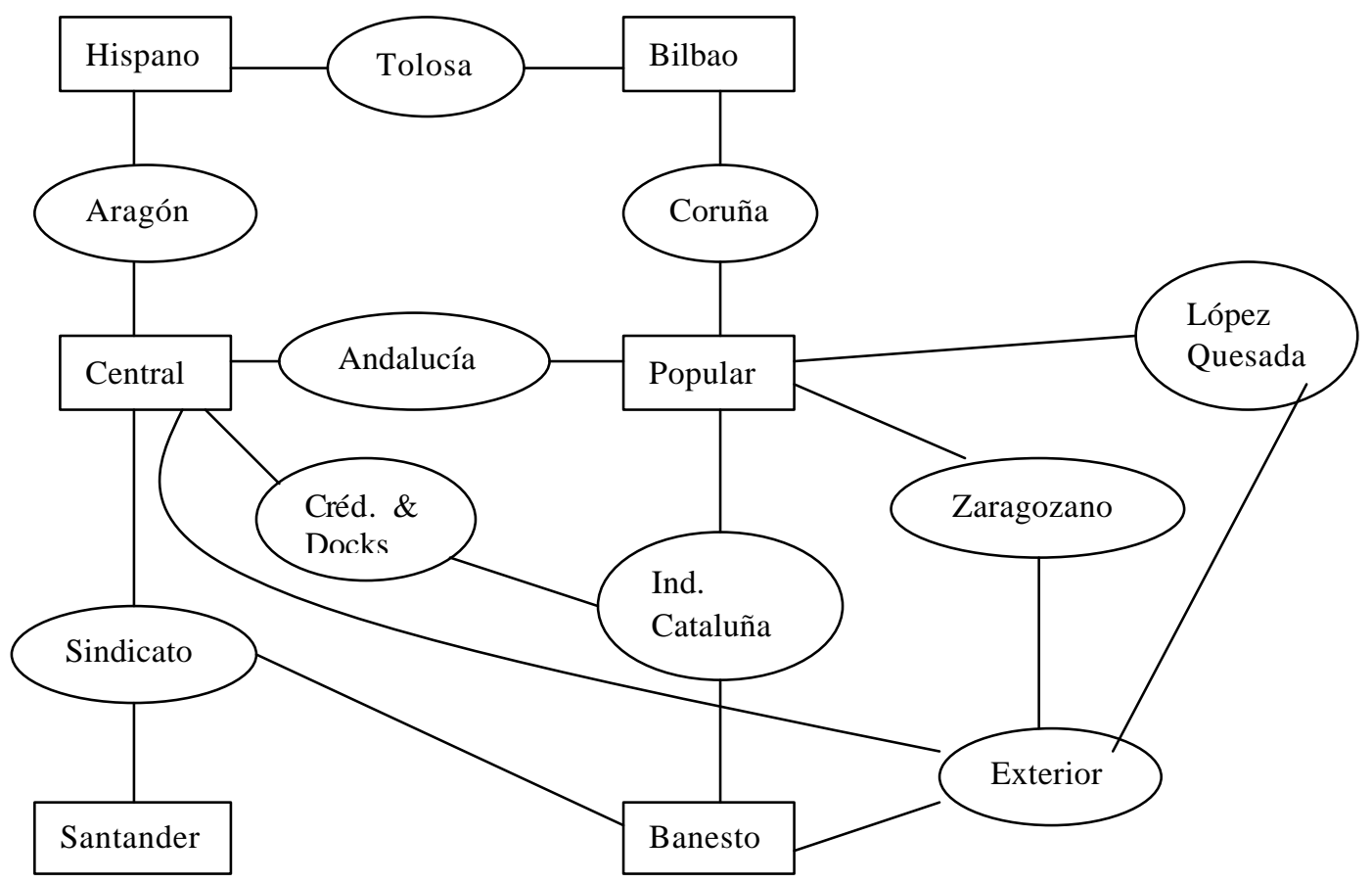

\section{Connections in 1980}

Without links: Bilbao, Santander, and Popular

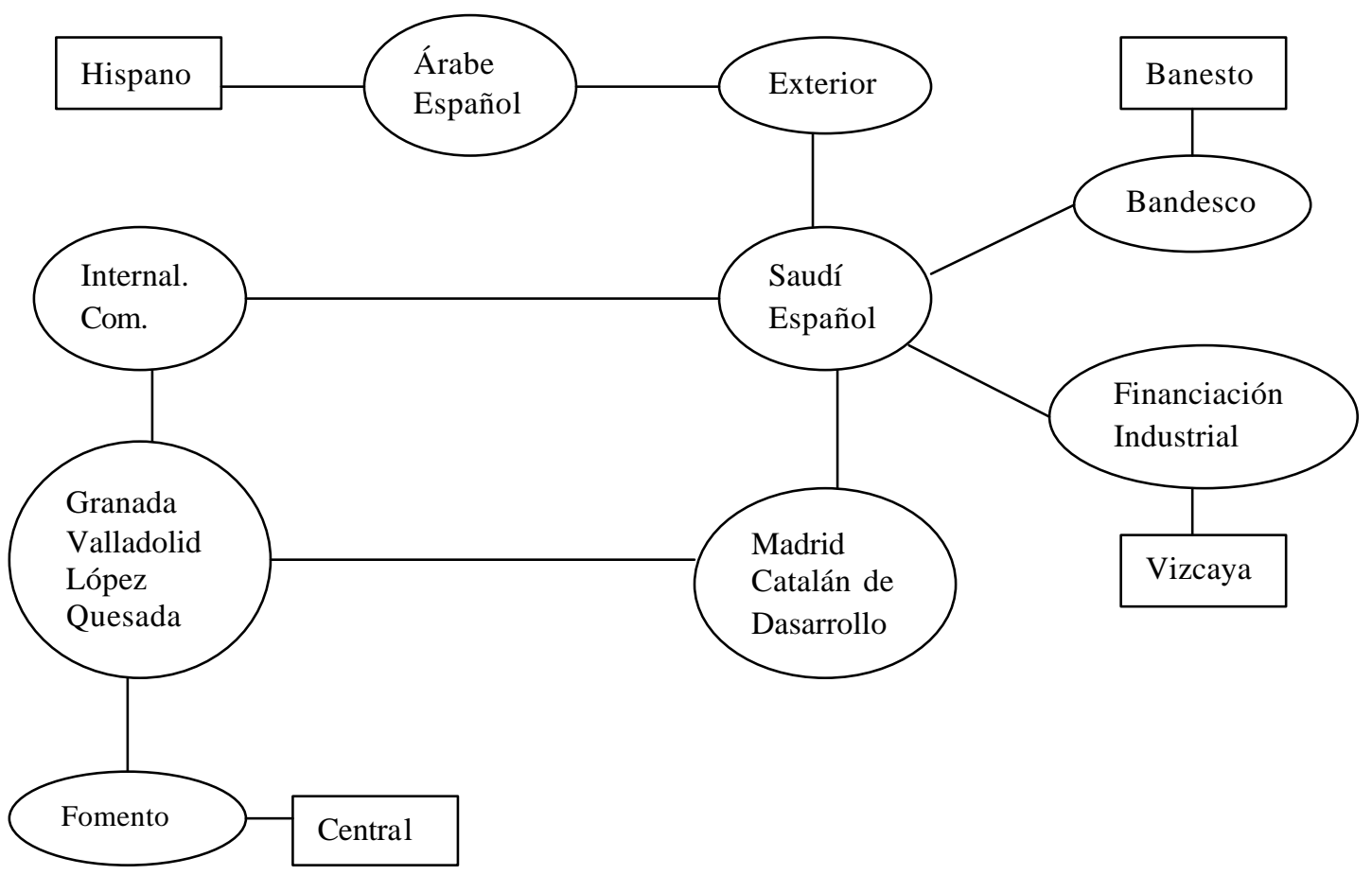




\section{Connections in 1985}

Without links: Bilbao, Central, and Popular

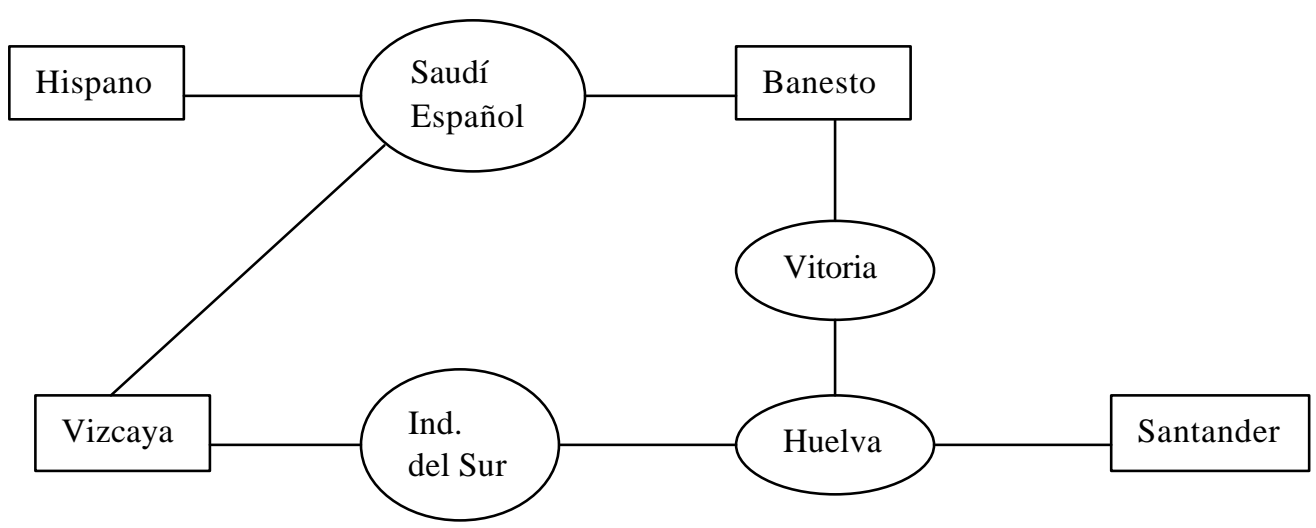

Connections in 1990

Without links: Hispano, Central, Santander, and Popular

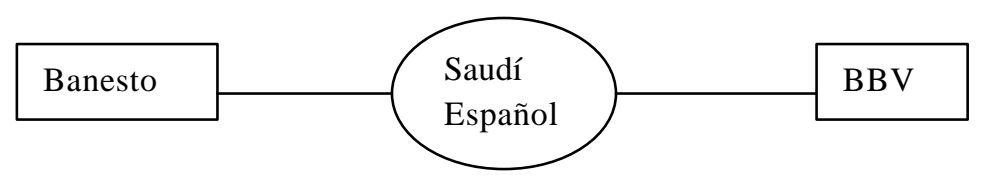




\section{Appendix 4: Lists of interlocking directors}

\section{Banco Hispano Americano}

1915 (Anuario Financiero y de Valores Mobiliarios, año I, 1916)

Banco Hispano Americano shared four directors with Banco Herrero: Amadeo Alvarez García, Celestino Alvarez García, Antonio Basagoiti -Hispano's chairman-, and Julian Cifuentes - Hispano's general manager-. Amadeo Alvarez García and Julian Cifuentes also sat in the board of Banco de Gijón.

1921 (Roldán-García Delgado, 1973, vol. 2, pg. 264-269)

According to Roldan-Garcia Delgado's diagrams, several common directors closely connected a set of banks with Banco Hispano Americano, clearly apart from other entities: Herrero, Gijón, and San Sebastián.

1925 (Anuario Financiero y de Sociedades Anónimas de España, año 1926)

Eight directors of Banco Hispano Americano sat in other banks' boards:

- Amadeo Alvarez García: Herrero and Gijón

- Celestino Alvarez García: Herrero

- Antonio Basagoiti y Arteta (chairman): Herrero and San Sebastian

- Julian Cifuentes y Fernández (general manager): Herrero and Gijón

- Adolfo Espinosa y Espinosa: San Sebastian

- Ignacio Herrero de Collantes, Marqués de Aledo: Herrero and San Sebastian

- Celedonio Noriega y Ruiz: San Sebastian

- Manuel de Taramona y Díaz de Entresotos: San Sebastian

1934 (Anuario Financiero y de Sociedades Anónimas de España, año 1935)

Eight directors connected Hispano Americano with Herrero, Gijón, and San Sebastián:

- Celestino Alvarez García: Herrero

- Amadeo Alvarez García, Conde del Real Agrado: Herrero, Gijón (chairman), and San Sebastian

- Federico Bernaldo de Quirós Argüelles: San Sebastian

- Ricardo García Trelles y Blanco: San Sebastian

- Guillermo Gil de Reboleño (vice-chairman): Herrero

- Ignacio Herrero de Collantes, Marqués de Aledo (chairman): Herrero (chairman), Gijón, and San Sebastian

- Andrés Moreno García (general manager): San Sebastian

- Celedonio Noriega Ruiz: San Sebastian 
1946 (Anuario Financiero y de Sociedades Anónimas de España, año 1947)

Seven directors of Banco Hispano Americano sat in the boards of Banco Herrero, Gijón, and San Sebastián:

- Amadeo Álvarez García, Conde del Real Agrado: San Sebastían and Gijón (chairman)

- Antonio Basagoiti Ruiz (vice-chairman): Herrero and Gijón

- Federico Bernaldo de Quirós Argüelles: San Sebastían

- Ignacio Herrero de Collantes, Marqués de Aledo (chairman): Herrero (chairman) and San Sebastían (vice-chairman)

- Andrés Moreno Garcia: San Sebastían

- Juan Manuel Torroba Goicoechea: San Sebastían

- Vicente Zaldo Arana: San Sebastían

From 1944 on, Banco Urquijo was firmly linked to Banco Hispano Americano. Following directors reflect this association, connecting banks that were members of Hispano and Urquijo's groups in the period before the civil war:

- Félix Escalas Chameni: Hispano Americano and Mercantil de Tarragona (chairman)

- Ignacio Herrero Garralda: Urquijo, Herrero (vice-chairman) and Gijón

- Miguel Mateu Pla-Deniel: Hispano Americano and Mercantil de Tarragona

Other connections:

- Ramón Barbat Miracle: Mercantil de Tarragona, Valls, and de la Propiedad

- José Ma Barrau Flaqué: Mercantil de Tarragona (vice-chairman) and Valls (chairman)

- Gumersindo Claramunt Pastor: Zaragozano (vice-chairman) and de la Propiedad

- Eduardo Comas Pérez Caballero: Zaragozano (manager) and de la Propiedad (vicechairman)

- Fco. de A. Costas Jové: Mercantil de Tarragona and Valls (administrator)

- Luis Homs Moncusí: Mercantil de Tarragona and Valls (vice-chairman)

- Santiago Trías Rumeu: Urquijo, Mercantil de Tarragona, and de la Propiedad

1961 (J. Muñoz, 1969, cap. 4, gráfico 1)

Muñoz's diagram displays multiple links with Herrero, Gijón, San Sebastián, and Urquijo. However, it does not appear Banco de Valls, that was connected with Hispano in 1946 and, again, in 1966. Banco Mercantil de Tarragona only appears linked with Hispano by just one interlocking director.

1966-67 (J. Muñoz, 1969, cap. 4, gráfico 3)

According to Muñoz's diagram, banks' members of Hispano Americano group would be the same we found in 1946, except that connections with banks Zaragozano and de la Propiedad get lost. 
Hispano Americano and Urquijo shared seven directors: Antonio Basagoiti Amézaga, Javier Benjumea Puigcerver, Pedro Durán Farrell, Pedro Gamero del Castillo (Hispano's chief executive and Urquijo's vice-chairman), Julio Hernández Rubio, Juan Antonio Ollero de la Rosa, and Luis de Usera y López-González (Hispano's chairman). Manual Otero Torres, secretary of Hispano Americano board, also sat in the board of Banco de Gijón.

1980 (Anuario Estadístico de la Banca Privada, año 1980)

Following Banco Hispano Americano directors linked this bank with Banco Hispano Industrial, Banco Urquijo, and Banco Mercantil de Tarragona:

- Alejandro Albert Solis (chief executive): Hispano Ind. (chairman), Urquijo, and Árabe Español

- José Manuel Arburúa Aspinza: Mercantil de Tarragona (vice-chairmane)

- Antonio Basagoiti Amézaga: Urquijo

- Antonio Basagoiti García-Tuñón (general manager): Hispano Ind. and Mercantil de Tarragona

- Javier Benjumea Puigcerver: Urquijo

- Jaime Carvajal Urquijo: Urquijo (chairman)

- Pedro Durán Farrell: Urquijo

- Pedro Gamero del Castillo (vice-chairman): Urquijo

- Francisco J. Hernández López-Gil (deputy general mana ger): Mercantil de Tarragona (chairman and chief executive)

- Julio Hernández Rubio: Urquijo

- Alberto Oliart Saussol ${ }^{21}$ : Urquijo

- Manual Otero Torres (secretary): Hispano Ind. (secretary) and Mercantil de Tarragona

- Jaime Soto López-Doriga (general manager): Hispano Ind.

- Francisco Torras Huguet (general manager): Hispano Ind. (vice-chairman)

- Francisco A. de Urquía y de la Yglesia: Mercantil de Tarragona

- Luis Usera Cano (general manager): Hispano Ind.

- Luis de Usera y López-González (chairman): Urquijo

Fernando Rubio Fernández, INI representative in Banco Árabe Español, connected this group with Banco Exterior.

1985 (Anuario Estadístico de la Banca Privada, año 1985)

Nine Hispano directors sat in the boards of other banks, mainly Urquijo-Unión and Hispano Industrial:

- José María Amusátegui de la Cierva (vice-chairman and chief executive): UrquijoUnión (chairman)

- Antonio Basagoiti García-Tuñón (general manager): Hispano Ind. and Urquijo-Unión

- José Capón Rey (general manager): Urquijo-Unión, Jerez (chairman) and Norte (chairman)

\footnotetext{
${ }^{21}$ Suspension from duty, because he was minister in the Spanish government.
} 
- Jaime Carvajal Urquijo: Hispano Ind. (chairman)

- Ramón Colao Caicoya (deputy general manager): Urquijo-Unión

- Miguel Geijo Baucells (general manager): Urquijo-Unión

- Emilio Novela Berlín (deputy general manager): Saudí Español

- Manual Otero Torres (secretary): Hispano Ind. (secretary), Jerez (secretary) and Norte (secretary)

- Benito Tamayo Hernáez (general manager): Mercantil de Tarragona (chairman) and Urquijo-Unión

Links through other banks:

- Ricardo Calvo Navarro: Jerez (chief executive) and Norte

- Francisco Navarro Chazarra: Jerez and Norte

- Benigno Rodríguez Rodríguez: Jerez and Norte

- Javier San Pío Sierra: Mercantil de Tarragona, Jerez, and Norte

- Ramón Zorrilla Zorrilla: Jerez and Norte

1990 (Anuario Estadístico de la Banca Privada, año 1990)

Only two Hispano Americano directors sat in other banks' boards of directors: José Manuel Arburúa Aspiunza was Banif's chairman (ex-Banco del Norte) and Manuel Otero Torres was Banco de Jerez's secretary. 


\section{Banco Urquijo}

1921 (Roldán-García Delgado, 1973, vol. 2, pg. 264-269)

In Roldán-García Delgado's diagram, Banco Urquijo's subsidiary companies appear clearly connected among them: Urquijo Catalán, Urquijo de Guipúzcoa, Urquijo Vascongado and Banco Minero Industrial de Asturias. Some directors connected these firms to Banco de Valencia and Banco Asturiano, so that they should be included into Urquijo's group. Other links joint them to Banco Hispano Colonial by means of one director of Urquijo Catalán and to Banesto's group by two or three directors. Two directors from Urquijo de Guipúzcoa were also in the board of Banesto and other director (who might be one of the formers) sat in the board of Banco Gijonés de Crédito, strongly linked by other connections to Banesto.

\section{5 (Anuario Financiero y de Sociedades Anónimas de España, año 1926)}

Twelve directors linked Urquijo's subsidiary companies, within which Banco Asturiano and Banco de Valencia should be included:

- Armando de las Alas Pumariño y Troncoso: Minero Industrial de Asturias (secretary), Asturiano de Industria y Comercio (managing director), and Valencia

- Luis de Echevarría: Urquijo Vascongado and Burgos

- Juan Tomás de Gandarias: Urquijo and Urquijo Vascongado (vice-chairman)

- Victoriano García San Miguel: Asturiano de Industria y Comercio and Valencia

- Valentín Ruiz Senen: Urquijo, Urquijo de Guipúzcoa, Urquijo Vascongado, Urquijo Catalán, and Minero Industrial de Asturias.

- Luis de Salazar y Zubia: Urquijo, Urquijo de Guipúzcoa, and Urquijo Vascongado

- Luis A. Sedó y Guichard: Urquijo and Urquijo Catalán

- José Tartière y Lenegre, Conde de Santa Bárbara de Lugones: Minero Industrial de Asturias (vice-chairman), Asturiano de Industria y Comercio (chairman), and Valencia (chairman)

- Enrique Urbina: Urquijo de Guipúzcoa and Urquijo Vascongado

- Estanislao Urquijo, Marqués de Urquijo: Urquijo (chairman) and Urquijo Catalán (chairman)

- Juan Manuel de Urquijo y Ussía: Urquijo (vice-chairman) and Minero Industrial de Asturias (chairman)

- Luis Urquijo y Ussia, Marqués de Amurrio: Urquijo, Urquijo de Guipúzcoa (chairman), and Urquijo Vascongado (chairman)

Apart from Banco de Burgos, several directors connected Urquijo's group to other banks. It was related with Banco Español de Crédito via two interlocking directors of Banco Urquijo de Guipúzcoa: Marqués de Alhucemas and Marqués de Cortina. The latter was then chairman of both Banesto and Banco Comercial Español, and vicechairman of Banco Gijonés de Crédito. Juan T. de Arteche was director of Banco de Bilbao, Baanco de Comercio and Banco Asturiano de Industria y Comercio. Finally, Ignacio Coll Portabella sat in the boards of Banco Hispano Colonial and Banco Urquijo Catalán. 
Following directors connected Banco Urquijo's subsidiaries and three other banks: Banco del Oeste de España, Mercantil de Tarragona and Banco de Valls:

- José María Barrau Flaqué: Mercantil de Tarragona (vice-chairman) and Valls (chairman)

- Francisco Blasi Cucurull: Mercantil de Tarragona y de Valls (managing director)

- Francisco Cubas Erice, Marqués de Fontalba: Urquijo and Urquijo de Guipúzcoa

- Félix Escalas Chamení: Urquijo Catalán (secretary) and Mercantil de Tarragona

- Juan Tomás de Gandarias: Urquijo and Urquijo Vascongado (vice-chairman)

- José Félix de Lequerica: Urquijo de Guipúzcoa and Urquijo Vascongado

- Juan Lladó Sánchez Blanco: Urquijo de Guipúzcoa and Minero Industrial de Asturias

- Damian Mateu Biza: Urquijo Catalán (vice-chairman) and Mercantil de Tarragona

- Manuel Miró Esplugas: Mercantil de Tarragona and Valls

- Pascual Pastor Carratalá: Minero Industrial de Asturias (secretary) and Oeste de

España

- Francisco Plana Sardá: Mercantil de Tarragona and Valls

- Valentín Ruiz Senen: Urquijo, Urquijo de Guipúzcoa, Urquijo Vascongado, Urquijo

Catalán, and Minero Industrial de Asturias (vice-chairman)

- Luis A. Sedó Guichard: Urquijo, Urquijo Catalán, and Mercantil de Tarragona

- Enrique Urbina: Urquijo de Guipúzcoa and Urquijo Vascongado

- Juan Manuel Urquijo y Ussia: Urquijo (vice-chairman), Urquijo de Guipúzcoa, Urquijo Vascongado, Minero Industrial de Asturias (chairman), and Oeste de España

- Luis Urquijo y Ussía, Marqués de Amurrio: Urquijo, Urquijo de Guipúzcoa (chairman), and Urquijo Vascongado (chairman)

- Estanislao Urquijo y Ussia, Marqués de Urquijo: Urquijo (chairman), Urquijo Catalán (chairman), and Mercantil de Tarragona (chairman)

Besides, Ignacio Coll Portabella, chairman of both Banco Hispano Colonial and Banca Arnús and member of the board of Crédito y Docks de Barcelona, sat also in the board of Banco Urquijo Catalán. 


\section{Banco Español de Crédito (Banesto)}

1915 (Anuario Financiero y de Valores Mobiliarios, año I, 1916)

Four directors of Banesto (León Cocagne - managing director-, Marqués de Alhucemas -chairman-, Marqués de Cortina -vice-chairman-, and Lucien Villars) were also directors of Banco Hipotecario.

1921 (Roldán-García Delgado, 1973, vol. 2, pg. 264-269)

In Roldán-García Delgado diagram, Banco Español de Crédito, Gijonés de Crédito and Comercial Español appeared linked by several directors. Besides, two or three directors of Banco Urquijo de Guipúzcoa sat in the boards of Banesto and Banco Gijonés de Crédito, and one or two directors of Banco Gijonés de Crédito connected Banesto group to Banco de Oviedo and Banco Castellano.

1925 (Anuario Financiero y de Sociedades Anónimas de España, año 1926)

Seven interlocking directors linked Banco de Oviedo, Gijonés de Crédito and Comercial Español to Banesto:

- Manuel de Argüelles: Comercial Español, Gijonés de Crédito, and Oviedo

- José Antonio Caicoya y Vigil-Escalera: Gijonés de Crédito (chairman) and Oviedo

- Marqués de Cortina: Español de Crédito (chairman), Comercial Español (chairman, Gijonés de Crédito (vice-chairman), and Urquijo de Guipúzcoa

- Pablo de Garnica: Español de Crédito, Comercial Español, and Oviedo

- Pedro Masaveu y Masaveu: Gijonés de Crédito and Oviedo

- César de la Mora y Abarca: Español de Crédito, Comercial Español (vice-chairman), and Gijonés de Crédito

- José Sela y Sela: Gijonés de Crédito and Oviedo

Besides, Marqués de Alhucemas and Marqués de Cortina linked Banesto to Banco Urquijo de Guipúzcoa.

1934 (Anuario Financiero y de Sociedades Anónimas de España, año 1935)

Banesto had no interlocking director this year.

1946 (Anuario Financiero y de Sociedades Anónimas de España, año 1947)

Three directors of Banesto sat in other banks' boards:

- José $\mathrm{M}^{\mathrm{a}}$ de Aguirre Gonzalo (manager): Guipuzcoano

- Pedro Barrié de la Maza (manager): Pastor (chairman and chief executive) 
- Epifanio Ridruejo Botija (chief executive): Guipuzcoano and Epifanio Ridruejo (vicechairman)

Other links through Banco Guipuzcoano:

- Agustín Brunet: Guipuzcoano (chairman) and Vitoria

- Ramón Machimbarrena: Guipuzcoano (chief executive) and Vitoria

- Rafael Villalonga Blanes: Guipuzcoano and Fomento Agrícola de Mallorca

1961 (J. Muñoz, 1969, cap. 4, gráfico 1)

According to Muñoz's diagram, banks connected to Banesto were the same as they were in 1946: Vitoria, Guipuzcoano and Epifanio Ridruejo.

1966-67 (J. Muñoz, 1969, cap. 4, gráfico 3)

In Muñoz's diagram, banks linked to Banesto in 1946 were Guipuzcoano, Vitoria and Epifanio Ridruejo. Besides, it appears Bandesco (Banco de Desarrollo Económico Español), the business bank founded by Banesto according to 1962 Banking Law. We also find some new links with Banca Garriga Nogués, which would remain in 1975. Banesto's group would be connected to other large banks through Sindicato de Banqueros, that connected it to Banco Central and Banco de Santander. Banca Garriga Nogués would link it with Banco Popular indirectly through Banco Industrial de Cataluña.

1975 (Anuario Estadístico de la Banca Privada, año 1975)

Following directors closely linked Banesto, Bandesco and Banco Guipuzcoano:

- José María Aguirre Gonzalo: Banesto (chairman), Bandesco (chairman), and Guipuzcoano (chairman)

- Gabriel de Garnica y Mansí: Banesto and Bandesco (chief executive)

- Pablo de Garnica y Mansí: Banesto (vice-chairman and chief executive) and Bandesco (vice-chairman and chief executive)

- Jaime Gómez-Acebo Modet: Banesto (honorary chairman) and Bandesco (honorary chairman)

- Juan Herrera Fernández: Banesto and Bandesco

- Pedro Masaveu Peterson: Banesto and Masaveu

- Julio de la Mora Garay: Banesto and Bandesco

- Epifanio Ridruejo Botija: Banesto, Bandesco, and Guipuzcoano

- José María Sainz de Vicuña y García Prieto: Banesto (chief executive) and Guipuzcoano

1980 (Anuario Estadístico de la Banca Privada, año 1980)

Following directors of Banesto connected it to Bandesco and Banco Guipuzcoano: 
- José María Aguirre Gonzalo (chairman): Bandesco (chairman) and Guipuzcoano (chairman)

- Jaime Argüelles Armada: Bandesco

- Gabriel de Garnica y Mansí: Bandesco (chief executive)

- Pablo de Garnica y Mansí (vice-chairman and chief executive): Bandesco (vicechairman)

- Juan Herrera Fernández: Bandesco

- Julio de la Mora Garay: Bandesco

- José María de Oriol y Urquijo: Bandesco

- Antonio Sáez de Mantagut: Guipuzcoano

- José María Sainz de Vicuña y García Prieto (general manager): Guipuzcoano

Jorge Brosa Palau, Bandesco's board secretary, also sat in the board of Banco Saudí Español.

1985 (Anuario Estadístico de la Banca Privada, año 1985)

Group formed by Banesto, Bandesco and Guipuzcoano held links with the association formed by Banco de Madrid, Catalán de Desarrollo and Garriga Nogués. Banco Saudí Español appears connected to both coalitions:

- Jaime Argüelles Armada: Banesto (vice-chairman) and Bandesco (vice-chairman)

- Gabriel de Garnica y Mansí: Banesto and Bandesco (chief executive)

- Pablo de Garnica y Mansí: Banesto (chairman) and Bandesco (chairman)

- Juan Herrera Fernández: Banesto and Bandesco

- José María López de Letona y Núñez del Pino: Banesto (vice-chairmane and chief executive), Catalán de Desarrollo (chairman), and Madrid (chairman)

- Cesar de la Mora Armada: Banesto and Garriga Nogués

- Antonio Sáez de Montagut: Banesto, Bandesco, and Guipuzcoano

- José María Sainz de Vicuña y García Prieto: Banesto and Guipuzcoano

- Francisco Luzuriaga Tobalina: Banesto and Guipuzcoano (vice-chairman)

- Jorge Brosa Palau: Bandesco (secretary) and Saudí Español

Trough other banks:

- Jacobo Argüelles Salaverría: Madrid and Vitoria (chairman)

- Fernando Castromil Sánchez: Madrid (secretary) and Catalán de Desarrollo (secretary)

- Félix Muelas Medrano: Madrid, Catalán de Desarrollo, and Saudí Español

- Enrique Ricart Vidal: Catalán de Desarrollo and Garriga Nogués (general manager)

- José Luis Fominaya Cisneros: Madrid (general manager) and Garriga Nogués

- Lliberat Hosta Romagosa: Madrid (general manager) and Garriga Nogués

1990 (Anuario Estadístico de la Banca Privada, año 1990)

Banesto had the following interlocking directors with Bandesco: Juan Belloso Garrido, Mario Conde Conde (chairman of both Banesto and Bandesco), Vicente Figaredo de la Mora, Ricardo Gómez-Acebo y Duque de Estrada (vice-chairman of both Banesto and Bandesco), Pedro Masaveu Peterson, Arturo Romaní Biescas and Antonio Sáez de Montagut (chairman of Banco General). 
Other links:

- Jorge Brosa Palau: Bandesco (secretary) and Saudí Español

- Enrique Lasarte y Pérez-Arregui: Banesto and Vitoria (chairman)

- Cesar de la Mora Armada: Desarrollo Económico Español and Garriga Nogués

- José María Sainz de Vicuña y García Prieto: Bandesco and Català de Crédit (chairman)

According to 1998 Banesto's Annual Report, 98 per cent of Banco de Madrid shares belonged to Banesto. Banco de Madrid was administrator of Banco de Albacete, Alicantino de Comercio and Peninsular. Through these banks, other links existed:

- José Luis Fominaya Cisneros: Madrid and Catalá de Crédit

- Esteban Lázaro Barreras: Madrid (deputy general manager) and Peninsular (administrator)

- Félix Muelas Medrano: Madrid, Català de Crédit, Saudí Español, and General

- Tomás Santoro García de la Parra: Madrid (secretary) and Català de Crédit (secretary) 


\section{Banco Central}

1921 (Roldán-García Delgado, 1973, vol. 2, pg. 264-269)

In Roldán-García Delgado's diagram, five directors connected Banco Central to Crédito de la Unión Minera. Besides, it was linked to other banks by only one director in each case: Banco de Santander, de Bilbao, Castellano and Cía. de Crédito Bancario. It is not possible to know whether any of these directors held some seats simultaneously.

1925 (Anuario Financiero y de Sociedades Anónimas de España, año 1926)

Five directors of Banco Central sat in other banks' boards:

- Mariano Baselga y Ramírez: Crédito de Zaragoza (first manager)

- Marceliano Isábal y Bada: Crédito de Zaragoza

- Arturo López Argüello: Castellano (managing director)

- Santos Vallejo y García: Castellano (chairman)

- José María Zorita y Díez (vice-chairman): Castellano (vice-chairman)

Besides, Miguel Rodríguez-Acosta y González de la Cámara also appears in the board of directors of Banco de Bilbao and de Comercio.

1934 (Anuario Financiero y de Sociedades Anónimas de España, año 1935)

Five directors of Banco Central connected it to other banks:

- Mariano Baselga Ramírez: Crédito de Zaragoza (managing director)

- Justino Bernard Valenzuela: Badalona (vice-chairman)

- Vicente Montal Cornelles: Hispano Colonial

- Samuel Ortiz Basualdo: Hispano Colonial

- Antonio P. Sasia (managing director): Hispano Colonial y Badalona

Through Banco Hispano Colonial, Banco Central was related to a group of Catalan banks:

- Eduardo María Buxaderas de la Cantera: Hispano Colonial (managing director), Banca Arnús (managing director), and Crédito y Docks de Barcelona

- Ignacio Coll Portabella: Hispano Colonial (chairman), Banca Arnús (chairman), Crédito y Docks de Barcelona, and Urquijo Catalán

- Miguel Drudis Biada: Crédito y Docks de Barcelona and Menorca (secretary)

- Francisco de P. Gambús Rusca: Arnús (managing director), Crédito y Docks de Barcelona (chairman) and Menorca (chairman)

- Eusebio López y Díaz de Quijano (Marqués de Lamadrid): Hispano Colonial and Banca Arnús (chairman)

- Julio Pauchet Hatrait: Arnús (managing director), Crédito y Docks de Barcelona and Menorca (vice-chairman)

- Luis de la Peña Braña: Hispano Colonial, de la Propiedad (vice-chairman) and LópezQuesada (vice-chairman) 
Nine directors of Banco Central sat in other banks' boards of directors:

- Pedro Armero y Manjón (Conde de Bustillo): Jerez (chairman)

- Vicente Boluda Martínez: Valencia

- José Galindo Gómez: Hispano Colonial, Crédito y Docks and Valencia

- José Ma Mayans y de Sequera (Conde de Trigona): Valencia (secretary)

- Salvador Millet Maristany: Popular Español

- Antonio Noguera Borona: Hispano Colonial and Valencia (chairman)

- Joaquín Reig Rodríguez: Hispano Colonial (manager) and Popular Español

- Darío Rumeu Freixa, Barón de Viver: Hispano Colonial (chairman) and Crédito y Docks

- Ignacio Villalonga Villalba (chairman): Hispano Colonial (manager), Crédito y Docks, and Valencia

Connections extended through Banco Hispano Colonial and Crédito y Docks:

- Emilio Botín Sáenz de Sautuola y López: Hispano Colonial (manager) and Santander (manager)

- Pedró Galí Marés: Hispano Colonial (manager) and Badalona

- Francisco de P. Gambús Rusca: Crédito y Docks (chairman) and Menorca (chairman)

- José Ma Milá y Camps, Conde del Montseny: Hispano Colonial (manager), Sindicato de Banqueros (secretary), and Banca Tusquets (chairman)

- Félix Millet Maristany: Hispano Colonial (manager) and Popular Español (chairman)

- Julio Pauchet Hatrait: Crédito y Docks and Menorca (vice-chairman)

1961 (J. Muñoz, 1969, cap. 4, gráfico 1)

In Muñoz's diagram, Banco Central appears linked to Banco Popular by several interlocking directorse and, besides, it was linked to Banco de Valencia and the pair Crédito y Docks-Agrícola Aragonés. Connection to Banco Santander went through Sindicato de Banqueros.

1966-67 (J. Muñoz, 1969, cap. 4, gráfico 3)

Banco Central held connections to Crédito y Docks, Banco de Valencia and Banco Agrícola Aragonés. In Muñoz's diagram, it also appears Banco de Fomento, that was Banco Central's industrial bank, and two small entities: Banco de Aragón and Banco de Úbeda. Links to other large banks went through Banco de Aragón (Banco Hispano Americano group), Banco de Andalucía and Banco Industrial de Cataluña (two links with Banco Popular) and Sindicato de Banqueros (Banesto and Santander).

1975 (Anuario Estadístico de la Banca Privada, año 1975) Valencia:

Following directors linked Banco Central, Banco de Fomento and Banco de - José María Aristrain Noaín: Central and Fomento 
- Alfonso Escámez López: Central (chairman and chief executive) and Fomento (chairman)

- Pedro Guerrero Jurado: Central and Fomento (vice-chairman and chief executive)

- Enrique Ramón Perdiguer: Valencia and Central

- Joaquín Reig Albiol: Fomento, Central and Valencia

- Joaquín Reig Rodríguez: Central (vice-chairman) and Valencia (chairman)

1980 (Anuario Estadístico de la Banca Privada, año 1980)

Following Banco Central's directors connected it to Banco de Valencia and Banco de Fomento.

- José María Aristrain Noaín: Fomento

- Alfonso Escámez López (chairman and chief executive): Fomento (chairman)

- Pedro Guerrero Jurado: Fomento (vice-chairman and chief executive)

- Joaquín Reig Albiol: Fomento y Valencia

- Joaquín Reig Rodríguez (vice-chairman): Valencia (chairman)

- Luis de Ussía y Gavaldá: Fomento

Epifanio Ridruejo Brieva (Banco de Fomento's general manager) was also director of Banco de Granada.

1985 (Anuario Estadístico de la Banca Privada, año 1985)

Banco Central, Banco de Fomento and Banco de Granada appear linked by several interlocking directors:

- José María Aristrain Noaín : Central and Fomento

- Juan Manuel Echevarría Hernández: Central (general manager) and Crédito e Inversiones (secretary)

- Alfonso Escámez López: Central (chairman and chief executive) and Fomento (chairman)

- Antonio Escámez López: Central and Valencia (vice-chairman)

- Javier Gil de Biedma y Vega de Seoane: Central, Fomento, and Granada

- Pedro Guerrero Jurado: Central and Fomento (vice-chairman)

- Tomás Parejo Camacho: Central and Granada (chairman)

- Joaquín Reig Albiol: Central, Fomento, and Granada

- Luis de Ussía y Gavaldá: Central and Fomento

Banco de Granada appears as the main link with Banco de Crédito e Inversiones and Banco Internacional de Comercio.

- Jesús Manuel Basanta Teijeiro: Granada, Crédito e Inversiones, and Internacional de Comercio

- Licinio de la Fuente y de la Fuente: Granada, Crédito e Inversiones, and Internacional de Comercio (vice-chairman)

- Juan González Lloret: Crédito e Inversiones (deputy general manager) and Noroeste (administrator)

- José Vázquez Riesco: Crédito e Inversiones, and Internacional de Comercio

- Eduardo Alconchel Romeo: Granada (administrator) and Sevilla (administrator) 
1990 (Anuario Estadístico de la Banca Privada, año 1990)

Seven directors of Banco Central sat in other banks' boards:

- Juan Manuel Echevarría Hernández (chief executive and secretary): Gallego

- Alfonso Escámez López (chairman): Fomento (chairman)

- Antonio Escámez López: Valencia (vice-chairman)

- Agustín Escámez Sánchez (sales manager): Gallego

- Javier Gil de Biedma y Vega de Seoane: Fomento and Granada

- Tomás Parejo Camacho: Granada (chairman)

- Luis de Ussía y Gavaldá: Fomento

Other connections:

- Jesús Manuel Basanta Teijeiro: Gallego, Granada, and Internacional de Comercio

- Licinio de la Fuente y de la Fuente: Gallego (chairman), Granada, and Internacional de Comercio (vice-chairman)

- Jaime Mairata Laviña: Gallego (secretary) and Internacional de Comercio (secretary)

- José Vázquez Riesco: Gallego and Internacional de Comercio 


\section{Banco de Bilbao}

1915 (Anuario Financiero y de Valores Mobiliarios, año I, 1916)

Twenty-seven directors of Banco de Bilbao also sat in Banco de Comercio's board of directors. Besides, Horacio de Echevarrieta also appears in Banco de Cartagena's board.

1921 (Roldán-García Delgado, 1973, vol. 2, pg. 264-269)

In Roldán-García Delgado's diagram, Banco de Bilbao only held one interlocking director with both Banco Central and Sindicato de Banqueros.

1925 (Anuario Financiero y de Sociedades Anónimas de España, año 1926)

Banco de Bilbao and Banco de Comercio shared twenty-seven directors and had the same chairman. Juan T. de Arteche also appears in the board of directors of Banco Asturiano de Industria y Comercio, that sould be included into Banco Urquijo group. Horacio de Echevarrieta also sat in the board of Banco Internacional de Industria y Comercio. Miguel Rodríguez-Acosta y González de la Cámara also appears in Banco Central board of directors.

1934 (Anuario Financiero y de Sociedades Anónimas de España, año 1935)

Four directors of Banco de Bilbao sat in other banks's boards:

- José de Aresti Ortiz: Comercio (chairman) and Asturiano de Industria y Comercio

- Julio Arteche Villabaso: Comercio and Asturiano de Industria y Comercio

- Victor de Chávarri Anduiza, Marqués de Triano: Asturiano de Industria y Comercio

- José Orbegozo Gorostegui: Castellano

1946 (Anuario Financiero y de Sociedades Anónimas de España, año 1947)

Eight directors of Banco de Bilbao sat in other banks' boards:

- Pedro de Ampuero Gandarias: Tolosa (vice-chairman)

- José Aresti Ortiz: Asturiano de Industria y Comercio

- Julio de Arteche Villabaso (chairman): Asturiano de Industria y Comercio

- Víctor Chávarri y Anduiza, Marqués de Triano: Asturiano de Industria y Comercio

- Alberto Delclaux Aróstegui: Castellano

- José Ma de Gondra y Lazúrtegui: Fomento Agrícola de Mallorca

- Julio Hernández Mendirichaga: Fomento Agrícola de Mallorca

- Lorenzo Hurtado de Saracho y Arregui: La Coruña

1961 (J. Muñoz, 1969, cap. 4, gráfico 1) 
Banco de Bilbao and Banco de Comercio shared several directors with Banco Asturiano y Banco Castellano. One director connected them to Banco de Tolosa (see Hispano Americano groups) and Banco de La Coruña (see Banco Central group).

1966-67 (J. Muñoz, 1969, cap. 4, gráfico 3)

In Muñoz's diagram, Banco de Bilbao association gathered the same banks as in 1961: Asturiano y Castellano. Besides, it appears Banco Industrial de Bilbao, Bilbao's industrial bank. Links to other large banks were through Banco de Tolosa (group of Hispano Americano) and Banco de La Coruña (group of Banco Popular).

1975 (Anuario Estadístico de la Banca Privada, año 1975)

Seven directors connected Banco de Bilbao to Banco Industrial de Bilbao: Rafael Acosta España (Bilbao's deputy chairman), José Manuel Delclaux y Barrenechea (Industrial de Bilbao's chairman), Arturo de Echevarría y Uribe, Faustino García Moncó y Fernández, Enrique de Guzmán y Martínez, Lorenzo Hurtado de Saracho y Arregui and Ramón de Icaza y Zabálburu.

1980 (Anuario Estadístico de la Banca Privada, año 1980)

Following directors connected Banco de Bilbao, Industrial de Bilbao and Banco de Comercio:

- José María Concejo Álvarez: Bilbao (secretary) and Huesca (chairman)

- José Manuel Delclaux y Barrenechea: Bilbao and Industrial de Bilbao (chairman)

- Arturo de Echevarría y Uribe: Bilbao and Industrial de Bilbao

- Faustino García Moncó y Fernández: Bilbao and Industrial de Bilbao

- Lorenzo Hurtado de Saracho y Arregui: Bilbao and Industrial de Bilbao

- Ramón de Icaza y Zabálburu: Bilbao and Industrial de Bilbao

- José María Tobar y Ochoa de Alda: Bilbao (general manager) and Comercio (secretary)

- Ceferino de Uríen y Goiricelaya: Bilbao and Industrial de Bilbao

- Rafael Acosta España: Comercio and Industrial de Bilbao

1985 (Anuario Estadístico de la Banca Privada, año 1985)

Twenty directors interlocked Banco de Bilbao and Banco Industrial de Bilbao. Three general managers of Banco de Bilbao sat simultaneously in Bilbao's board and Banca Mas Sardá board. Besides, two directors of Banco de Bilbao connected it to other banks:

- José María Concejo Álvarez (secretary): Industrial de Bilbao (secretary) and Huesca (chairman)

- Fernando Martínez de Bedoya y Martínez-Carande (general manager): Comercio, Extremadura (chairman), Oeste (chairman), and Promoción de Negocios (chairman) 
Finally, Pedro Fontana García, who was Banca Mas Sardà's general manager, also sat in Sindicato de Banqueros' board of directors.

1990 (Anuario Estadístico de la Banca Privada, año 1990)

Following directors of BBV connected it to other banks:

- Eduardo de Aguirre Alonso-Allende: Privanza (vice-chairman)

- Luis Javier Bastida Ibargüen (general manager): Privanza

- José María Concejo Álvarez (secretary): Comercio (board adviser) and Industrial de Bilbao (chairman)

- Ángel Díez de Ulzurrun Bergera (general manager): Comercio (vice-chairman) and Meridional

- José María Echevarría Arteche (general manager): Industrial de Bilbao

- Carlos Gorría Cortazar (deputy chairman): Banca Catalana (vice-chairman)

- José Javier Gúrpide Huarte (2 ${ }^{\text {nd }}$ vice-chairman): Comercio (chairman)

- Federico Lipperheide Wicke: Saudí Español (vice-chairman)

- Victor Manuel Menéndez Millán (general manager): Crédito Canario, Meridional, and

Occidental

- Juan Antonio Palacios Raufast (general manager): Occidental

- Javier Prado Urquijo: Privanza (chairman)

- José Aureliano Recio Arias (chief executive): Meridional (chairman) and Finanzia (chairman)

- Álvaro Rengifo Calderón (deputy chairman): Extremadura (chairman)

- Armin Rose Thate (deputy chairman): Saudí Español

- Alfredo Sáenz Abad ( $1^{\text {st }}$ vice-chairman): Banca Catalana (chairman)

- Joaquín Sánchez Izquierdo (general manager): Occidental and Finanzia

- José Luis Segimón Escobedo (secretary): Banca Catalana (secretary), Industrial de

Cataluña (secretary), Industrial del Mediterráneo (secretary), and Occidental (chairman)

Other connections:

- José Borrue Asensio: Industrial de Bilbao, Meridional, Occidental, and Crédito y Ahorro (chairman)

- Fernando Quintana Fernández: Crédito Canario (secretary), Crédito y Ahorro (secretary), Meridional (secretary) and Occidental

- Fernando Martínez de Bedoya y Martínez de Carande: Crédito Canario (chairman) and Promoción de Negocios (chairman)

- Alfredo Sáenz Abad: Industrial de Cataluña (chairman) and Industrial del

Mediterráneo (chairman)

- Catalana de Cobros y Factoring, S.A. (CACYF): Ba rcelona, Industrial de Cataluña and Industrial del Mediterráneo

- Mobiliario Mat, S.A.: Barcelona, Industrial de Cataluña and Industrial del Mediterráneo

- Sociedad Europea de Arrendamiento, S.A. (SEASA): Barcelona and Industrial de Cataluña

- Rentequipo, S.A.: Barcelona and Industrial del Mediterráneo 


\section{Banco de Vizcaya}

1915 (Anuario Financiero y de Valores Mobiliarios, año I, 1916)

Felipe Ugalde connected Banco de Vizcaya to Banco Guipuzcoano.

1921 (Roldán-García Delgado, 1973, vol. 2, pg. 264-269)

Banco de Vizcaya does not appear in Roldán-García Delgado's diagrams, so that it is supposed that it had no interlocking directors with any other bank.

1925 (Anuario Financiero y de Sociedades Anónimas de España, año 1926)

Banco de Vizcaya had no interlocking directors with any other bank.

1934 (Anuario Financiero y de Sociedades Anónimas de España, año 1935)

Banco de Vizcaya had no interlocking directors with any other bank.

1946 (Anuario Financiero y de Sociedades Anónimas de España, año 1947)

Banco de Vizcaya had no interlocking directors with any other bank.

1961 (J. Muñoz, 1969, cap. 4, gráfico 1)

In Muñoz's diagram, Banco de Vizcaya appears connected to Banco Hispano Suizo and Banco de Vilella by only one director.

1966-67 (J. Muñoz, 1969, cap. 4, gráfico 3)

Banco de Vizcaya does not appear in Roldán-García Delgado's diagrams, so that it is supposed that it had no interlocking directors with any other bank.

1975 (Anuario Estadístico de la Banca Privada, año 1975)

Six directors connected Banco de Vizcaya to Banco de Financiación Industrial (ex-Hispano Suizo): Julio Arce García (Vizcaya's deputy chairman and general manager), José Raimundo Basabe y Manso de Zúñiga (Banco de Financiación Industrial's general manager), Pedro de Careaga y Basabe (Vizcaya's honorary chairman), Manuel M. de Gortázar y Landecho (Banco de Financiación Industrial's chairman), Carlos Mendoza Gimeno (Banco de Financiación Industrial vice-chairman) and Ignacio de Muguruza y Madariaga. 
1980 (Anuario Estadístico de la Banca Privada, año 1980) Industrial:

Following directors connected Banco de Vizcaya to Banco de Financiación

- Julio Arce García: Vizcaya (deputy chairman and general manager) and Financiación Industrial

- José Raimundo Basabe y Manso de Zúñiga: Vizcaya and Financiación Industrial (vice-chairman)

- Pedro de Careaga y Basabe: Vizcaya (honorary chairman) and Financiación Industrial

- Manuel M. de Gortázar y Landecho: Vizcaya and Financiación Industrial (chairman)

- Carlos Mendoza Gimeno: Vizcaya and Financiación Industrial

- Ignacio de Muguruza y Madariaga: Vizcaya and Financiación Industrial

- Joaquín Nebreda de Miguel: Vizcaya (general manager), Financiación Industrial, and Crédito Comercial (chairman)

- José Luis Serrano Lizarralde: Vizcaya (gene ral manager) and Financiación Industrial

- Pedro Toledo Ugarte: Vizcaya (chief executive) and Financiación Industrial

- Juan Manuel Urgoiti López de Ocaña: Vizcaya (general manager) and Financiación Industrial

Armin Rose Thate, who was Banco de Financiación Industrial's general manager, also sat in Banco Saudí Español board of directors.

1985 (Anuario Estadístico de la Banca Privada, año 1985)

Seven directors sat simultaneously in the boards of directos of Banco de Vizcaya and Banco de Financiación Industrial. Besides, Federico Lipperheide Wicke was director of Banco de Vizcaya and Banco Saudí Español's vice-chairman. Other six directors connected them to two sets of banks, headed by Banca Catalana and Banco Occidental:

- Ángel Díez de Ulzurrun Bergera: Vizcaya (general manager), Financiación Industrial, Crédito Comercial, and Meridional

- José Luis Segimón Escobedo: Vizcaya (secretary), Banca Catalana (secretary), Industrial de Cataluña (secretary), Industrial del Mediterráneo (secretary), Occidental (secretary), Crédito y Ahorro (secretary), and Crédito Comercial (chairman)

- Juan Manuel Urgoiti López de Ocaña: Vizcaya (dir. gral.), Financiación Industrial, Banca Catalana, Occidental (chairman), and Crédito Canario (chairman)

- Julio Gilsanz Arrola: Financiación Industrial (general manager) and Industrial del Sur (chairman)

- Antonio Negre Villavecchia: Financiación Industrial and Banca Catalana (chairman)

- José Ignacio Berroeta Echevarría: Financiación Industrial and Banca Catalana

Other connections:

- José Borrue Asensio: Occidental, Crédito y Ahorro (chairman), Crédito Comercial, and Meridional 
- Fernando Quintana Fernández: Crédito Canario (secretary), Crédito Comercial (secretary), Industrial del Sur (secretary), and Meridional (secretary)

- Victor Manuel Menéndez Millán: Occidental, Crédito Comercil, Crédito Canario, and Meridional

- José Antonio Sáenz-Azcunaga Usandizaga: Occidental (vice-chairman) and Meridional (chairman)

- Juan Antonio Palacios Raufast: Occidental and Crédito y Ahorro

- Francisco Luzón López: Occidental (general manager) and Industrial del Sur

- Mario Ezquerra Arnalot: Banca Catalana and Crédito Canario

- Javier Peralte de las Heras: Huelva and Industrial del Sur

- Alfredo Sáenz Abad: Banca Catalana (general manager), Barcelona (chairman), Industrial de Cataluña (chairman), and Industrial del Mediterráneo (chairman)

- Catalana de Cobros y Factoring S.A. (CACYF): Barcelona, Industrial de Cataluña, and Industrial del Mediterráneo

- Mobiliario Mat S.A.: Barcelona, Industrial de Cataluña, and Industrial del Mediterráneo

- Rentequipo S.A.: Barcelona, Industrial de Cataluña, and Industrial del Mediterráneo

- Sociedad Español de Arrendamiento S.A. (SEASA): Barcelona, Industrial de Cataluña, and Industrial del Mediterráneo 


\section{Catalan banks before the civil war}

1915 (Anuario Financiero y de Valores Mobiliarios, año I, 1916)

Francisco de P. Gambús, who was Banco Arnús' deputy director, sat also in the board of directors of Banco de Sabadell. Enrique Turull y Comadrán was director of Banco de Sabadell and Crédito y Docks de Barcelona. José Gari y Cañas held three seats: in Banco de Barcelona, Banca Arnús-Garí and Sindicato de Banqueros de Barcelona. Francisco Sert i Badía was director of Banco de Barcelona and Banco Hispano Colonial.

1921 (Roldán-García Delgado, 1973, vol. 2, pg. 264-269)

Connections among Catalan banks appear rather confused, but they may be assembled in three coalitions, that were related between them via Cía. de Crédito Bancario and Sindicato de Banqueros:

- Banco De Cataluña headed the first association (Banco De Reus and Banco De Tortosa). At the same time, it held one interlocking director with Banca Marsans, three with Cía. de Crédito and two with Sindicato de Banqueros.

- Banca Arnús, Crédito y Docks, Banco de Menorca and Banco de Sabadell formed the second group. These banks mantained up to six links with Cía. de Crédito and one more with Sindicato de Banqueros.

- Banco Hispano Colonial, Banca Arnús-Garí and Banco de Barcelona would have formed a third association, but they were weakly linked. They would have been connected to Urquijo Catalán by one interlocking director and held two other links with Cía. de Crédito. Although it does not appear in Roldán-García Delgado's diagram, they linked Banco Hispano Colonial to Banca López Bru in the text (p. 267).

1925 (Anuario Financiero y de Sociedades Anónimas de España, año 1926)

Two groups may be distinguished in the Catalan banking system and one interlocking director related them. Banco de Cataluña led the first group:

- Evaristo Fábregas Pamies: Cataluña, Reus (manager) and Tortosa (manager)

- Tomás Mallol y Bosch: Cataluña and Comercial de Tarragona

- Eduardo Recaséns y Mercadé: Cataluña, Reus (manager), Tortosa (manager), and Sindicato de Banqueros (secretary)

- Arturo Suqué Sucona: Cataluña and Reus.

The second group was formed by a set of banks linked to Banca Arnús and Crédito y Docks:

- Jaime Carner Romeu: Crédito y Docks de Barcelona (chairman) and Arnús (vicechairman)

- Francisco de P. Gambús Rusca: Crédito y Docks de Barcelona, Arnús (deputy director), Sabadell and Menorca (chairman)

- Juan Marcet: Comercial de Tarrasa (chairman) and Crédito y Docks de Barcelona

- Julio Pauchet: Crédito y Docks de Barcelona, Arnús (manager) and Menorca (vicechairman). 
Other Catalan banks held interlocking directorates, but only two cases shared more than a director. First, we have a pair formed by Banco de Préstamos y Descuentos and Banco Comercial de Barcelona:

- Ernesto Carpi Gentilli: Internacional de Industria y Comercio, Comercial de Barcelona (general manager), and Préstamos y Descuentos

- Luis Escayola Vallhonrat: Comercial de Barcelona and Préstamos y Descuentos

- Fernando Mercé González: Comercial de Barcelona (vice-chairman) and Préstamos y

Descuentos

- Francisco Piella Surerol: Comercial de Barcelona and Préstamos y Descuentos

Second, there is the group formed by Banco Internacional de Industria y Comercio, Banca Marsans and Banco Comercial de Tarrasa.

- José Guardiola Roca: Marsans y Comercial de Tarrasa

- Juan Miquel Banús: Marsans (manager) and Internacional de Industria y Comercio (administrator)

- Francisco Salvans Armengol: Marsans and Comercial de Tarrasa

- Juan Ventosa y Calvell: Marsans (chairman) and Internacional de Industria y Comercio (vice-chairman)

Other Catalan banks connections:

- Ignacio Coll Portabella: Hispano Colonial y Urquijo Catalán

- Luis Figueras Dotti: Marsans (deputy manager) and Sindicato de Banqueros

- Juan Antonio Gamazo y Abarca, Conde de Gamazo: Internacional de Industria y Comercio, Hispano Colonial, and S. A. Arnús-Garí (chairman)

- Jorge Garí Gimeno: Préstamos y Descuentos, Sindicato Banqueros, and S. A. ArnúsGarí

- José Garriga Nogués y Roig: Garriga Nogués (vice-chairman) and Sindicato Banqueros

- Juan Antonio Güell, Conde de Güell: Hispano Colonial and López Brú (vicechairman)

- Horacio de Echevarrieta: Banco de Bilbao and Banco Internacional de Industria y Comercio

- Marqués de Quijano: Hispano Colonial and Mercantil

1934 (Anuario Financiero y de Sociedades Anónimas de España, año 1935)

Two groupss of Catalan banks may be found this year, apart from those related to Banco Hispano Colonial (see Banco Central connections). One of this coalitions would have been around Banca Marsans:

- Miguel Barella Arraut: Marsans and Comercial de Barcelona (secretary)

- Vicente Boluda Martínez: Valencia and Castellón

- Manuel Casanova Llopis: Valencia and Castellón (vice-chairman)

- Luis Escayola Vallhonrat: Marsans (general manager), Comercial de Barcelona (general manager), and Internacional de Industria y Comercio

- José Escuder Alcaide: Valencia (manager) and Internacional de Industria y Comercio (manager)

- José Galindo Gómez: Valencia and Castellón

- José Geis Bosch: Marsans and Comercial de Tarrasa 
- José Guix Ribas: Marsans and Comercial de Tarrasa

- Luis Jover Castells: Marsans and Comercial de Barcelona

- Juan Miquel Banús: Marsans (manager), Internacional de Industria y Comercio, and Sindicato de Banqueros

- Vicente Noguera Bonora: Valencia (chairman), Castellón (chairman), and Internacional de Industria y Comercio (chairman)

- Francisco de A. Ripoll Fortuño: Marsans (chairman) and de la Propiedad

- Francisco Salvans Armengol: Marsans (manager) and Comercial de Tarrasa (chairman)

- Juan Salvans Pascual: Marsans (vice-chairman) and Internacional de Industria y Comercio

- Ignacio Villalonga Villalba: Valencia and Internacional de Industria y Comercio

It subsisted some connections between banks that had been linked to Banco de Cataluña:

- Salvador Floriach Arnau: Tortosa and Palafrugell

- Pedro Torra Closa: Reus and Tortosa

Finally, Jorge Garí Gimeno, who was S.A. Arnús-Garí's vice-chairman, was also Sindicato de Banqueros' secretary. 


\section{Banco de Santander}

1961 (J. Muñoz, 1969, cap. 4, gráfico 1)

In Muñoz's diagram, Banco Santander held links by only one director with Banco Soler and Sindicato de Banqueros (see groups of Banco Central).

1966-67 (J. Muñoz, 1969, cap. 4, gráfico 3)

Banco de Santander shared several directors with Banco Comercial para América (ex-Banco Soler y Torra) and Banco Intercontinental (Bankinter). Banco de Santander was related with other groups by a director of Sindicato de Banqueros (see groups of Banco Central and Banesto).

1975 (Anuario Estadístico de la Banca Privada, año 1975)

Following directors linked Banco Santander, Comercial para America and Intercontinental Español (Bankinter):

- Luis Bergé Abarca: Santander, Comercial para América and Bankinter

- Emilio Botín-Sanz de Sautuola y García de los Ríos: Santander $\left(2^{\text {nd }}\right.$ vice-chairman and general manager) and Bankinter

- Emilio Botín-Sanz de Sautuola y López: Santander (chairman) and Bankinter (chairman)

- Jaime Botín-Sanz de Sautuola y García de los Ríos: Santander and Bankinter (chief executive)

- Santiago Corral y Pérez: Santander and Bankinter

- Joaquín Chapaprieta Ornstein: Santander and Comercial para América

- Ives C. Lamarche: Comercial para América and Bankinter

- Peter M. Nelson: Bankinter and Comercial para América (vice-chairman)

- Eduardo Ruiz de Huidobro y Alzurena: Santander and Bankinter

- Alvin C. Rice: Comercial para América (chairman) and Bankinter

- Pablo Tarrero Rivero: Santander ( $1^{\text {st }}$ vice-chairman and chief executive) and Bankinter

- Ramón Zapater: Bankinter and Comercial para América

1980 (Anuario Estadístico de la Banca Privada, año 1980)

Following directors related Banco de Santander, Bankinter and Bank of America:

- Luis Bergé Abarca: Santander (general manager) and Bankinter

- Emilio Botín-Sanz de Sautuola y García de los Ríos: Santander ( $2^{\text {nd }}$ vice-chairman and chief executive), Bankinter, Comercial Español, and Jover

- Jaime Botín-Sanz de Sautuola y García de los Ríos: Santander and Bankinter (chief executive)

- Emilio Botín-Sanz de Sautuola y López: Santander (chairman) and Bankinter (chairman)

- Santiago Corral y Pérez: Santander and Bankinter 
- Joaquín Chapaprieta Ornstein: Santander and Bankinter

- Juan Secades González-Camino: Santander (general manager) and Bankinter

- Pablo Tarrero Rivero: Santander ( $1^{\text {st }}$ vice-chairman and chief executive) and Bankinter

- Louis d'Aurelle de Paladines: Bankinter and Bank of America (general manager)

- Juan Manuel Forn: Bankinter and Bank of America (Bank of America New York representative and deputy general manager)

- Mont. E. McMillen: Bankinter and Bank of America (chairman)

- Vaden M. Yelton: Bankinter and Bank of America (vice-chairman)

1985 (Anuario Estadístico de la Banca Privada, año 1985)

Ten directors connected Banco de Santander, Intercontinental Español and Santander de Negocios:

- Luis Bergé Abarca: Santander (general manager), Intercontinental Español and Santander de Negocios

- Emilio Botín-Sanz de Sautuola y García de los Ríos: Santander (2 ${ }^{\text {nd }}$ vice-chairman and chief executive) and Intercontinental Español

- Jaime Botín-Sanz de Sautuola y García de los Ríos: Santander and Intercontinental Español (chief executive)

- Emilio Botín-Sanz de Sautuola y López: Santander and Intercontinental Español (chairman)

- Santiago Corral y Pérez: Santander and Intercontinental Español

- Joaquín Chapaprieta Ornstein: Santander and Intercontinental Español

- José Luis Díaz Fernández: Santander and Comercial Español

- Alberto Folch Rusiñol: Santander and Santander de Negocios

- Atilano Matilla Gómez: Santander (secretary) and Comercial Español

- Juan Secades González-Camino: Santander and Intercontinental Español

Three other directors linked this group to other entities:

- Rafael Alonso Botín: Santander and Jover (vice-chairman)

- Rodrigo Echenique Gordillo: Santander de Negocios (secretary), Condal, and Huelva

- Vaden M.Yelton: Intercontinental Español and Bank of America (vice-chairman)

Banco Condal and Banco de Huelva shared five directors more:

- Fernando de Chávarri Zapatero: Condal (secretary) and Huelva (general manager and secretary)

- Francisco de la Herrán Matorras: Condal (chairman) and Huelva (chairman)

- José Ramón Meléndez Martínez-Agulló: Condal and Huelva

- Eugenio Posada Puntero: Condal and Huelva

- Martín Rivas Fernández: Condal, Huelva, and Vitoria

1990 (Anuario Estadístico de la Banca Privada, año 1990)

Ten directors of Banco de Santander sat in the boards of other banks:

- Rafael Alonso Botín (manager): Santander de Negocios y Jover

- Emilio Botín-Sanz de Sautuola y García de los Ríos (chairman): Santander de Negocios (chairman) and Bankinter (vice-chairman) 
- Jaime Botín-Sanz de Sautuola y García de los Ríos (vice-chairman): Bankinter (chairman)

- José María Carballo Cotanda (manager): Santander de Negocios (chief executive)

- Joaquín Chapaprieta Ornstein: Bankinter

- Rodrigo Echenique Gordillo (chief executive): Santander de Negocios

- Carlos Gerardo García Rodríguez (manager): Jover

- Julio Novo Polidura (manager): Santander de Negocios

- Juan Rodríguez Inciarte (manager): Santander de Negocios (vice-chairman)

- Juan Secades González-Camino: Bankinter

Banco Jover linked Santander's association with Banco Condal and Banco de Huelva:

- José Ignacio Uclés Romero: Jover (secretary), Condal (secretary), and Huelva

- Pedro Insaurriaga Gamo: Condal and Huelva

- José Ramón Meléndez Martínez-Agulló: Condal and Huelva

- Eugenio Posada Puntero: Condal and Huelva 


\section{Banco Popular}

1966-67 (J. Muñoz, 1969, cap. 4, gráfico 3)

Banco Popular held links with Banco de Andalucía and Banco de Salamanca, as in 1961 (see Banco Central cluster). Through Banco de Andalucía, Banco Popular was connected to Banco de Huelva that was owned by RUMASA. Besides, now it appears Banco Europeo de Negocios, an industrial bank. Banco Popular was related with groups of other large banks through Banco de La Coruña (Bilbao), Banco de Andalucía (Central), Banco Industrial de Cataluña (Banesto and Central), and through ZaragozanoLópez Quesada-Exterior with Banesto and Central.

\section{5 (Anuario Estadístico de la Banca Privada, año 1975)}

Following directors connected Banco Popular to Banco Europeo de Negocios and other banks:

- Luis Butragüeño Ramos: Popular (general manager) and Europeo de Negocios

- Juan Manuel Fanjul Sedeño: Popular (vice-chairman) and Castilla (chairman)

- Gonzalo Fernández de la Mora y Mon: Popular and Europeo de Negocios

- José María Lage Arenas: Popular (deputy chief executive) and Europeo de Negocios

- Miguel Nigorra Oliver: Popular, Europeo de Negocios, and Crédito Balear (chairman)

- Fernando de Solís y Atienza: Popular and Andalucía (chairman)

- Rafael Termes Carrero: Popular (chief executive) and Europeo de Negocios

- Javier Valls Taberner: Popular ( $1^{\text {st }}$ vice-chairman $)$ and Europeo de Negocios

- Pedro Valls Taberner: Popular (consejero secretario ejecutivo de la presidencia) and Europeo de Negocios

- Gilbert Barre: Societe Generale de Banque en Espagne (vice-chairman and chief executive) and Europeo de Negocios

1980 (Anuario Estadístico de la Banca Privada, año 1980) mainly:

Following directors connected Banco Popular to Banco Popular Industrial,

- Luis Butragüeño Ramos: Popular (general manager) and Popular Ind. (secretary)

- Gonzalo Fernández de la Mora y Mon: Popular and Popular Ind.

- Manuel Laffón de la Escosura: Popular, Popular Ind. (chairman) and Andalucía (vicechairman)

- Miguel Nigorra Oliver: Popular and Crédito Balear (chairman)

- Rafael Termes Carrero: Popular (chief executive) and Popular Ind.

- Javier Valls Taberner: Popular ( $1^{\text {st }}$ vice-chairman) and Popular Ind.

- Pedro Valls Taberner: Popular (secretario ejecutivo de la presidencia) and Popular Ind.

- Juan Moya García: Andalucía (secretary) and Huelva (secretary) 
Five people sat in the boards of Banco Popular and Banco Popular Industrial, simultaneously: Manuel Laffón de la Escosura, José Oliva Marín, Rafael Termes Carrero $^{22}$, Javier Valls Taberner and Pedro Valls Taberner. Three other directors connected Banco Popular to other entities:

- Juan Manuel Martín Martín: Exportación (deputy general manager)

- Miguel Nigorra Oliver: Crédito Balear (chairman)

- Fernando de Solis de Atienza: Andalucía (chairman)

1990 (Anuario Estadístico de la Banca Privada, año 1990) directors:

Banco Popular held links with other entities by the following interlocking

- Manuel Laffón de la Escosura (chief executive): Popular Industrial

- Miguel Nigorra Oliver: Crédito Balear (chairman)

- Fernando de Solis de Atienza: Andalucía (chairman)

- Rafael Termes Carrero: Popular Industrial

- Javier Valls Taberner (chairman): Popular Industrial

- PedroValls Taberner: Popular Industrial

\footnotetext{
${ }^{22}$ In suspension from duty because he was the chairman of the Spanish Banking Association (AEB).
} 


\section{Other relations}

1915 (Anuario Financiero y de Valores Mobiliarios, año I, 1916)

* Four directors of Banco Asturiano de Industria y Comercio (Armando de las Alas Pumariño -general manager-, Victoriano García San Miguel, José Tartiere and Marcelino Trapiello) also appears in the boards of Banco de Valencia. Besides, Juan T. de Arteche linked Banco Asturiano to Crédito de la Unión Minera.

* Banco Riojano and "La Agrícola" shared three directors: Fermín Goñi (La Agrícola general manager), Juan Irurzun, and Francisco Urcola.

* The chairman of Fomento Agrícola Industrial y Comercial (Luchmajor, Mallorca), Juan Puig Fullana, also appears in the board of Banco de Soller (Mallorca).

* Lorenzo Pons sat in the boards of Banco de Menorca and Crédito Mercantil de Menorca. Juan Vidal held seats in two boards of directors: Banco de Menorca and Crédito Mercantil de Menorca.

1925 (Anuario Financiero y de Sociedades Anónimas de España, año 1926)

* Banco de Santander shared three directors with Banco de Torrelavega.

* Banco Español en París connected many small and regional banks:

- Roque de Alday: Tolosa (chairman)

- Juan Bautista Alfaro: Vitoria (chairman)

- Marqués de Arenas: Ávila (chairman)

- Joaquín Beunza y Redín: La Vasconia

- Marqués de Cabanes: Sindicato de Banqueros

- Alberto Carrión (vice-chairman): Aragón (general manager)

- Alberto Elósegui (chairman): Vitoria

- José García Sánchez: Zaragozano (chairman)

- José Maestre Zapata: Internacional de Industria y Comercio

- Demetrio de los Mozos (secretary): Guipuzcoano (secretary)

* Two directors of La Vasconia sat in other banks' boards:

- Cecilio Azcárate y Lana: Aragón

- Tomás Garmendía y Biurrun: Internacional de Industria y Comercio

* There were also some connections between banks in the Balearic Islands:

- Conde de Torre Saura: Crédito Mercantil de Menorca and Comercial de Ciudadela (vice-chairman)

- Juan J. Vidal y Mir: Crédito Mercantil de Menorca (vice-chairman) and Fomento Agrícola de Mallorca (secretary) 
* Banco Guipuzcoano and Banco Vitoria shared two directors, including Guipuzcoano's general manager, Victor Artola.

* Banco de Santander and Banco de Torrelavega shared three directors, including general manager (Emilio Botín) and vice-chairman (Saturnino Briz Larín) of both banks.

* Chairman of Banco de Irún was also vice-chairman of Banco Mercantil e Ind.

* Banco de Aragón and La Vasconia had one interlocking director.

* Banco Zaragozano and Banco de la Propiedad shared two directors, including Zaragozanos' vice-chairman.

* Banks of the Balearics held the following connections:

- Juan Mir Company: Fomento Agrícola de Mallorca and Fomento Agrícola Industrial y Comercial (secretary).

- Conde de Torre Saura: Crédito Mercantil de Menorca (vice-chairman) and Comercial de Ciudadela (vice-chairman).

- Juan J. Vidal y Mir: Fomento Agrícola de Mallorca (manager) and Crédito Mercantil de Menorca (chairman).

1946 (Anuario Financiero y de Sociedades Anónimas de España, año 1947)

* Banco de de Aragón and Banco de Tortosa shared two directors: Miguel Ma Azcárate Irurita and Joaquín Bau Nolla, chairman of Banco de Tortosa.

* Jose Garriga Nogués y Garriga Nogués, Marqués de Cabanes, was the chairman of Banca Garriga Nogués and Sindicato de Banqueros.

1961 (J. Muñoz, 1969, cap. 4, gráfico 1)

* In Muñoz's diagram, it appears the following associations of small banks:

- Mas Sardà-Comercial Transatlántico-Sindicato

- Garriga Noguès-Sindicato

- Jover-Sindicato

- Mercantil e Industrial-Crédito Aragonés-Málaga-Canarias

1966-67 (J. Muñoz, 1969, cap. 4, gráfico 3)

* Coalitions interlocked by more than one director would have been:

- Crédito Navarro-La Vasconia, that were also connected to Banco de Aragón (see Banco Central groups).

- Madrid-Catalán de Desarrollo, that were connected to Banco Central through Crédito y Docks. 
- Sevilla-Meridional, linked to Banco Popular through Banco de Huelva.

- Atlántico-Unión Industrial Bancaria, connected to Banco Central.

- Banca Catalana-Industrial de Cataluña-Comercial Transatlántico. Banco Industrial de Cataluña linked them with groups of Banesto, Central and Popular.

- Mercantil-Canarias-Aragonés del Crédito, linked to Banco Exterior.

1975 (Anuario Estadístico de la Banca Privada, año 1975)

* Following directors of Banco Atlántico sat in other banks' boards:

- Guillermo Bañares Martí (chief executive): Comercial de Cataluña (chairman)

- Pablo Bofill de Quadras: Unión Industrial Bancaria (vice-chairman)

- José Ferrer Bonsoms (vice-chairman): Unión Industrial Bancaria (chairman)

- José María Molero y Aguero: Comercial de Cataluña

- Casimiro Molins Ribot (chairman): Unión Industrial Bancaria

* Following directors of Banca Catalana sat in other banks' boards:

- Francisco Cabana Vancells (secretary): Gerona (chairman) and Ind. de Cataluña

- Raimon Carrasco Azemar (general manager): Ind. de Cataluña (vice-chairman)

- Juan Casablancas Bertrán: Mercantil de Manresa (chairman)

- Jordi Pujol Soley: Ind. de Cataluña

- Olegario Soldevilla Godó: Ind. de Cataluña

- José Luis Vilaseca Guasch: Mercantil de Manresa

* Eight directors connected Banco de Madrid to Banco Catalán de Desarrollo: Claudio Boada Villalonga ( $2^{\text {nd }}$ vice-chairman of Catalán de Desarrollo), Jaime Castell Lastortras (chairman of both banks), Jaime Castell Mercader (Banco Catalán de Desarrollo's deputy chairman), Carlos Corpas Gutiérrez-Zorrilla, Francisco Luis Gamón Boldoba (Banco de Madrid's chief executive), Juan Pujol Isern (Banco Catalán de Desarrollo's chief executive), Juan Rosell Codinachs (vice-chairman of both banks) and Ramón Sardá Pérez-Bufill.

* Three directors connected Banco Mercantil de Tarragona and Banco de Valls: Francisco J. Hernández López-Gil (Mercantil de Tarragona's vice-chairman and chief executive), Juan Sanmartí Arderiu (Valls' vice-chairman) and Eudaldo Tarre Arnau (secretary of both boards).

* Three directors linked Banco Ibérico to Banco de Finanzas: Arturo Fierro Viña (Ibérico's vice-chairman and Finanzas' chairman and chief executive), Basilio Freire Caeiro de Matta y Luis Rodríguez Viña (Ibérico's chief executive).

* Two directors connected Banco de Granada to Banco de Crédito Comercial: J. Manuel Rodriguez-Acosta Carlstrom (Crédito Comercial's chairman and Granada's chief executive), Miguel Rodriguez-Acosta Carlstrom (Crédito Comercial's chief executive and Granada's chairman).

* Finally, next people shared two boards of directors:

- Miguel Capuz Pons: Cantábrico (secretary) and Valladolid

- Vicente Castellano Sabater: Exportación and Barcelona (vice-chairman)

- Santiago Guillén Moreno: Albacete (chairman) and Norte (legal adviser) 
- Fernando Labastida Nicolau: Comercial Español and Industrial del Mediterráneo (secretary)

- Arturo Mas Sarda Laguna: Sindicato de Banqueros (vice-chairman) and Mas Sarda (1 ${ }^{\text {st }}$ vice-chairman)

- Domingo Romera Alcázar: Peninsular and Condal

- Francisco Tardío Cabello: Sindicato de Banqueros and Vitoria (chairman)

1980 (Anuario Estadístico de la Banca Privada, año 1980)

* Banca Catalana headed a set of banks (Industrial del Mediterráneo, Industrial de Cataluña, de Barcelona and Banco de Gerona) by the next interlocking directors:

- Antonio Armengol Arnau: Banca Catalana (deputy general manager) and Industrial del Mediterráneo

- Francisco Cabana Vancells: Banca Catalana (secretary), Gerona (chairman) and Ind. de Cataluña

- Raimon Carrasco Azemar: Banca Catalana (chairman) and Industrial de Cataluña (vice-chairman)

- Salvador Casanovas Martí: Banca Catalana and Barcelona (chairman)

- Francisco Constans Ros: Banca Catalana (general manager) and Industrial del Mediterráneo

- Antonio Forrellad Solá: Banca Catalana, Industrial de Cataluña (vice-chairman) and Industrial del Mediterráneo (chairman)

- Juan Martí Mercadal: Banca Catalana and Industrial de Cataluña

- Ramón Monforte Navalón: Banca Catalana (deputy general manager) and de Barcelona

- Andrés Ribera Rovira: Banca Catalana (vice-chairman) and Industrial de Cataluña (chairman)

- Martí Rosell Barbé: Banca Catalana and Industrial de Cataluña

- Oleguer Soldevilla Godó: Banca Catalana and Industrial de Cataluña

- José Luis Vilaseca Guasch: Banca Catalana, Gerona (vice-chairman), and Valladolid

- Ventura Garcés Brusés: Industrial de Cataluña (secretary) and Industrial del Mediterráneo (secretary)

* Banco de Granada, de Valladolid and Banca López Quesada held several interlocking directors among them and with other entities:

- Javier Escauriaza Urioste: Granada and Pastor (deputy general manager)

- José Ramón García Arango: Granada, López Quesada (vice-chairman and general manager), and Valladolid (chief executive)

- José Giménez de Almenara: Granada and Madrid (general manager)

- Ignacio González Cagigas: Granada (secretary), Depósitos (secretary), López Quesada (secretary and legal adviser), and Valladolid (secretary)

- Juan Luis Iglesias Prada: Granada (vice-chairman) and López Quesada (vicechairman)

- Aristóbulo de Juan de Frutos, Corporación Bancaria representative: Granada (chairman and chief executive), López Quesada (chairman), Meridional, Rural y Mediterráneo, and Valladolid (chairman)

- José Luis López de Garayo y González de Betolaza: Granada and Unión

- Enrique Piñel López: March (secretary) and Valladolid

- Lorenzo de Zavala Richi: Internacional de Comercio and López Quesada 
* Epifanio Ridruejo Brieva, Banco de Granada's director, was also general manager of Banco de Fomento (see Banco Central's clusters).

* Another coalition was formed by Banco de Madrid and Banco Catalán de Desarrollo: José María Amusátegui de la Cierva, Jacobo Argüelles Salaverría, Claudio Boada Villalonga (chairman of both banks), Fernando Castromil Sánchez (secretary of both banks), Pablo de Garnica Gutiérrez, José María López de Letona y Núñez del Pino y Félix Muelas Medrano (he was also director of Banco Saudí Español).

* Banco Exterior and Banco Cantábrico appear associated by the following interlocking directors: Rafael Martínez Cortiña (Exterior's chief executive), José Luis Mora Mallo (Exterior's general manager) y Fermín Zelada de Andrés Moreno (chairman of both banks). Another two directors of Banco Exterior connected it to other entities: Francisco Novela Berlín to Banco Saudí Español and Fernando Rubio Fernández to Banco Árabe Español.

* Three directors connected Banco Occidental to Banco Comercial Occidental: Gregorio Diego Jiméne z (chairman of both banks), Oscar García Siso (Occidental's chief executive and Comercial Occidental's vice-chairman) and Juan Antonio Llopis Martínez.

* Two directors connected Banco de Levante to Nuevo Banco: José Miguel Garrigues Walker (chairman of both banks) and José Antonio Madruga Casado (vice-chairman of both banks).

* Another pairs:

- José Ferrer Bonsoms: Atlántico (vice-chairman) and Unión (chairman)

- Joaquín López Ayuso: Atlántico (general manager) and Comercial de Cataluña

- José María Recuero Albizu: Atlántico and Jerez (secretary)

- Juan Ros Petit: Atlántico and Condal (secretary)

- Juan Gich Bech de Careda: Condal and Peninsular (chairman)

- Vicente Castellano Sabater: Exportación and Barcelona

- Santiago Guillén Moreno: Albacete (chairman) and Extremadura (legal adviser)

- Patricio Pemán Medina: Extremadura (chairman) and Industrial del Sur (legal adviser)

- Manuel Igea Gil: Abel Matutes Torres and Trelles

- Juan Lladó y Fernández-Urrutia: Internacional de Comercio (general manager) and Saudí Español

- Javier de la Rosa Martí: Catalán de Desarrollo and Garriga Nogués (vice-chairman)

1985 (Anuario Estadístico de la Banca Privada, año 1985)

* Following directors of Banco Exterior sat in other banks' boards:

- Miguel Boyer Salvador (chairman): Alicante (chairman)

- Gerardo Burgos Belascoain: Simeón

- Joaquín Casuso Posada (general manager): Atlántico y Simeón (vice-chairman)

- Ignacio Domínguez Urquijo: Árabe Español

- Ramón Fernández de la Mora y Álvarez (general manager): Alicante ( $1^{\text {st }}$ vicechairman), Cantábrico, Atlántico y Simeón 
- Rafael Martínez Cortiña (vice-chairman and chief executive): Atlántico (vicechairman) y Simeón (chairman)

- José Luis Mora Mallo (general manager): Cantábrico

- Ríos Pérez. Calixto (general manager): Simeón

Other connections:

- José Luis Alonso Pérez: Alicante and Cantábrico

- Abdulla A. Saudi: Atlántico (chairman) and Árabe Español (chairman)

- Salem A. Zenaty: Atlántico and Arabe Español (deputy general manager)

- José Sartorious Álvarez de Bohorques: Atlántico and Árabe Español (vice-chairman)

* Banco Zaragozano and Banco de Toledo shared ten directors. One of them, Sergio García-Orcoyen Tormo, was also general manager in Spain of Algemene Bank Nederland, N.V..

* Three directors connected Banco Herrero to Banco Herrero Inversión.

* Four directors of Banca March connected this bank to other three:

- Alfredo Lafita Pardo (vice-chairman): Asturias (chairman) and Natwest-March (chief executive)

- Juan March Delgado: Natwest-March and Progreso (chairman)

- Enrique Piñel López (secretary): Asturias and Natwest-March (secretary)

- Cristobal Rodríguez Pizá (managing director): Asturias

* Other pairs of banks:

- Javier Abad Hernando: Árabe Español (deputy general manager) and Exportación

- Luis Vañó Martínez: Árabe Español (general manager) and Exportación (chairman)

- GESPAR, S.A.: Castilla (administrator) and Galicia (administrator)

1990 (Anuario Estadístico de la Banca Privada, año 1990)

* Eleven directors of Banco Exterior sat in other banks' boards:

- José Luis Alonso Pérez (general manager): Gestión and Inversión Financiera

- Joaquín Casuso Posada (general manager): Atlántico and Gestión e Inversión Financiera (chairman)

- Ignacio Gil Vallejo (general manager): Alicante ( ${ }^{\text {st }}$ vice-chairman), Exterior Directo, Gestión e Inversión Financiera, and Simeón (chairman)

- Rafael Gómez Perezagua: Árabe Español (vice-chairman)

- Francisco Luzón López (chairman): Atlántico (vice-chairman) and Exterior Internacional (chairman)

- Jesús María Martín Herrero (secretary): Alicante and Simeón

- José Ramón Muinelo Aguirre (general manager): Alicante, Gestión e Inversión Financiera and Exterior Directo

- Calixto Ríos Pérez (general manager): Exterior Internacional

- José Ignacio Rivero Pradera (chief executive): Alicante (chairman), Atlántico, and

Exterior Directo (chairman)

- Luis Daniel Sanz Suárez: Árabe Español

- Fernando Vivar Mira (secretary): Exterior Directo (secretary), Exterior Internacional (secretary), and Simeón (secretary) 
Through Banco Exterior Directo there were other links:

- Rosa Alegría Íñiguez (chief executive): Simeón

- Luis Estrada Parra: Atlántico and Simeón

- Marco Antonio García Pérez: Alicante

- José Ramón Jouve Jiménez: Alicante, Gestión e Inversión Financiera and Simeón

- Gabriel Terroba Gutiérrez: Alicante, Gestión e Inversión Financiera and Simeón

Interlocks through other banks:

- Ángel María Bengoa Zabala: Gestión e Inversión Financiera and Simeón

- Manuel Planelles Notario: Gestión e Inversión Financiera and Simeón (general manager)

- José Quevedo Quevedo: Gestión e Inversión Financiera and Simeón

- Amador Villar Santos: Gestión e Inversión Financiera and Simeón

- José Ataz Hernández: Alicante (secretary) and Gestión e Inversión Financiera (secretary and legal adviser)

- Antonio de Santiago Vila: Alicante and Gestión e Inversión Financiera

- Abdulmohsen Yousef AlHunaif: Árabe Español (vice-chairman) and Atlántico

- José María Chimeno Chillón: Atlántico (general manager) and Exportación (chairman)

- Abdulla A. Saudi: Árabe Español (chairman) and Atlántico (chairman)

* National Westminster Bank held following links with other banks:

- Edwin Herbert Fairman: Asturias and National Westminster Bank, P.L.C. (chief executive)

- Rafael Martínez Cortiña: Asturias (chairman) and Natwest-España (chairman)

- Nigel Paul Imison: Asturias and Natwest España (deputy chairman)

- Miguel Vizcaíno Calderón: Asturias and Natwest España (secretary)

* Deutsche Bank, A.G. and Banco Comercial Transatlántico had three interlocking directors: Pedro Fisterbusch (Deutsche Bank and Comercial Transatlántico manager), Alberto Ignacio Veciana Verges (Comercial Transatlántico manager) and Anton Walter Batzler (Comercial Transatlántico manager).

* Banco Zaragozano and Banco de Toledo shared four directors: Juan Luis Arrieta Barrenechea (Zaragozano's general manager), Eugenio Domingo Solans, Jaime Requeijo González (Toledo's chairman), Eduardo Solans Abadías (Toledo and Zaragozano's vice-chairman). Besides, one director of Banco Zaragozano, Juan Llopart Pérez, also sat in the board of Sindicato de Banqueros.

* Banco de Sabadell and Sabadell Multibanca shared four directors: Alberto Borrás Ferre (Sabadell's deputy general manager), Buenaventura Garriga Brutau (Sabadell's vice-chairman and Sabadell Multibanca's chairman), José Permanyer Cunillera (Sabadell's deputy general manager and Sabadell Multibanca's general manager) and Francisco Vallejo Vallejo (Sabadell deputy general manager).

* Three directors of Banca March also appear in the board of Banco Urquijo: Simón J. Galmés Cerdó (Banca March vice-chairman), José Carlos March Delgado (Banca March and Urquijo chairman) and Jaime Prohéns Mas. Through Banco Urquijo, there were more connections: José Antonio Casaus Martín (Banco del Progreso chief 
executive), Juan March Delgado (Banco del Progreso chairman) and Leopoldo Rodes Castañé (Banco del Progreso vice-chairman).

* Finally, Cía de Gestión y Administración, S.A. (GESPAR, S.A.) appears as administrator of Banco de Castilla, Banco de Galicia and Banco Vasconia.

* Guy de Jacquelot connected Credit Lyonnais to Banco Comercial Español. 